\title{
Lee-Yang zeros and the complexity of the ferromagnetic Ising model on bounded-degree graphs
}

\author{
Pjotr Buys ${ }^{1}$, Andreas Galanis ${ }^{2}$, Viresh Patel ${ }^{3}$ and Guus Regts ${ }^{4}$ \\ ${ }^{1}$ Korteweg de Vries Institute for Mathematics, University of Amsterdam, Postbus 94248, 1090 GE Amsterdam, \\ The Netherlands; E-mail: pjotr.buys@gmail.com. \\ ${ }^{2}$ Department of Computer Science, University of Oxford, OX1 3QD, UK; E-mail: andreas.galanis@cs.ox.ac.uk. \\ ${ }^{3}$ Korteweg de Vries Institute for Mathematics, University of Amsterdam, Postbus 94248, 1090 GE Amsterdam, \\ The Netherlands; E-mail: v.s.patel@uva.nl. \\ ${ }^{4}$ Korteweg de Vries Institute for Mathematics, University of Amsterdam, Postbus 94248, 1090 GE Amsterdam, \\ The Netherlands; E-mail: guusregts@gmail.com \\ An extended abstract of this article has appeared in the proceedings of SODA 2021.
}

Received: 22 January 2021; Accepted: 5 January 2022

2020 Mathematics Subject Classification: Primary - 68W25; Secondary - 05C31, 37F10

\begin{abstract}
We study the computational complexity of approximating the partition function of the ferromagnetic Ising model with the external field parameter $\lambda$ on the unit circle in the complex plane. Complex-valued parameters for the Ising model are relevant for quantum circuit computations and phase transitions in statistical physics but have also been key in the recent deterministic approximation scheme for all $|\lambda| \neq 1$ by Liu, Sinclair and Srivastava. Here, we focus on the unresolved complexity picture on the unit circle and on the tantalising question of what happens around $\lambda=1$, where, on one hand, the classical algorithm of Jerrum and Sinclair gives a randomised approximation scheme on the real axis suggesting tractability and, on the other hand, the presence of Lee-Yang zeros alludes to computational hardness. Our main result establishes a sharp computational transition at the point $\lambda=1$ and, more generally, on the entire unit circle. For an integer $\Delta \geq 3$ and edge interaction parameter $b \in(0,1)$, we show \#P-hardness for approximating the partition function on graphs of maximum degree $\Delta$ on the arc of the unit circle where the Lee-Yang zeros are dense. This result contrasts with known approximation algorithms when $|\lambda| \neq 1$ or when $\lambda$ is in the complementary arc around 1 of the unit circle. Our work thus gives a direct connection between the presence/absence of Lee-Yang zeros and the tractability of efficiently approximating the partition function on bounded-degree graphs.
\end{abstract}

\section{Introduction}

The Ising model is a classical model from statistical physics that arises in multiple sampling and inference tasks across computer science. The model has an edge interaction parameter $b$ and a vertex parameter $\lambda$, known as the external field. For a graph $G=(V, E)$ (all graphs considered in this article are finite), configurations of the model are all possible assignments of two spins,+- to the vertices of $G$. Each configuration $\sigma: V \rightarrow\{+,-\}$ has weight $\lambda^{\left|n_{+}(\sigma)\right|} b^{\delta(\sigma)}$, where $n_{+}(\sigma)$ is the set of vertices that get the spin + under $\sigma$ and $\delta(\sigma)$ is the number of edges that get different spins. ${ }^{1}$ The partition function

\footnotetext{
${ }^{1}$ The parametrisation of the Ising model in terms of $\delta(\sigma)$ follows the closely related works [35, 42]; if instead the model is defined in terms of the number of edges with the same spins, the edge interaction parameter $1 / b \in(1, \infty)$ is obtained, whose logarithm corresponds to the inverse temperature in the physics literature.
}

(C) The Author(s), 2022. Published by Cambridge University Press. This is an Open Access article, distributed under the terms of the Creative Commons Attribution licence (https://creativecommons.org/licenses/by/4.0/), which permits unrestricted re-use, distribution, and reproduction in any medium, provided the original work is properly cited. 
is the aggregate weight of all configurations; that is,

$$
Z_{G}(\lambda, b)=\sum_{\sigma: V \rightarrow\{+,-\}} \lambda^{\left|n_{+}(\sigma)\right|} b^{\delta(\sigma)} .
$$

In this article, we consider the problem of approximating the partition function when $b \in(0,1]$, known as the ferromagnetic case, and when the parameter $\lambda$ is in the complex plane. Complex parameters for the Ising model have been studied in the computation of probability amplitudes of quantum circuits; see, for example, $[12,36,8]$. Somewhat surprising - and this is one of the main motivations behind this work - complex parameters are also fundamental in understanding the complexity of approximation even for real-valued parameters.

In particular, many of the recent advances on the development of approximation algorithms for counting problems have been based on viewing the partition function as a polynomial of the underlying parameters in the complex plane and using refined interpolation techniques from [1,39] to obtain fully polynomial time approximation schemes (FPTAS; see Subsection 1.1 below for the technical definition), even for real values [23, 24, 33, 5, 2, 34, 44, 42, 41]. The bottleneck of this approach is establishing zerofree regions in the complex plane of the polynomials, which in turn requires an in-depth understanding of the models with complex-valued parameters. This framework of designing approximation algorithms aligns with the classical statistical physics perspective on phase transitions, where zeros in the complex plane have long been studied in the context of phase transitions (see, e.g., [29, 25]), and several of these classical results have recently been used to obtain efficient approximation algorithms ([35, 39]).

In particular, the celebrated Lee-Yang theorem [30] says that, when regarding the partition function of the ferromagnetic Ising model as a polynomial in the external field parameter $\lambda$, all of its zeros, referred to as Lee-Yang zeros, lie on the unit circle in the complex plane. (These Lee-Yang zeros have actually been observed in quantum experiments [40].) The Lee-Yang theorem was recently used by Liu, Sinclair and Srivastava [35] to obtain an FPTAS for approximating the partition function for values $\lambda \in \mathbb{C}$ that do not lie on the unit circle. This result can be viewed as a derandomisation of the Markov chain-based randomised algorithm by Jerrum and Sinclair [26] for $\lambda>0$ (see also [22, 11]), solving a longstanding problem. ${ }^{2}$

As noted in [35, Remark p. 290], the 'no-field' case $|\lambda|=1$ is unclear, since, on the one hand, we have the algorithm by [26] for $\lambda=1$ and, on the other hand, it is known that Lee-Yang zeros are dense on the unit circle. The density picture was further explored in [42] for graphs of bounded maximum degree $\Delta$, by establishing for each $b \in(0,1)$ a symmetric arc around $\lambda=1$ on the unit circle where the partition function does not vanish for all graphs of maximum degree at most $\Delta$ and showing density of the Lee-Yang zeros on the complementary arc. See also [10] for the density result.

In this article, we resolve the complexity picture of the ferromagnetic Ising model. We show that for graphs of maximum degree $\Delta$, approximately computing the partition function is \#P-hard, ${ }^{3}$ on the arc of the unit circle where the Lee-Yang zeros are dense. See Theorem 1 for a precise statement of our main results. Since on the complementary arc there exists an FPTAS, by the results of [42] (in combination with [1, 39]), this gives a direct connection between hardness of approximation and the presence of Lee-Yang zeros. Combined with the results of [26, 35], our work therefore classifies the complexity of approximating the partition function of the ferromagnetic Ising model on the complex plane.

It should be noted that the existence of zeros does not imply hardness in a straightforward manner. ${ }^{4}$ We obtain the connection between the Lee-Yang zeros and computational complexity via tools from

\footnotetext{
${ }^{2}$ Notably, the correlation decay approach, which also yields deterministic approximation algorithms and was key in the full classification of antiferromagnetic 2-spin systems [31, 45, 46, 16], somewhat surprisingly does not perform as well for ferromagnetic systems; see [21] for the state-of-the-art on this front.

${ }^{3}$ Roughly, \#P is the counting version of problems in NP; see, for example, [47] for details.

${ }^{4}$ For example, the graphs in [42] whose partition function is shown to be zero are trees and these can be clearly detected in polynomial time. More generally, it is hard to imagine a construction of graphs with vanishing partition function which can directly yield hardness. In any case, our results, following the framework of $[20,6,7]$, show hardness for a relaxed version of the problems where zeros do not need to be detected, making all of these considerations irrelevant.
} 
complex dynamical systems. The partition function on trees naturally gives rise to a dynamical system; cf. Subsection 2.1. Both the hardness of approximating the partition function as well as the density of the Lee-Yang zeros originate from chaotic behavior of the dynamics, while normal behavior is linked to absence of zeros and hence the existence of efficient approximation algorithms [42].

Our work falls into the broader context of showing how zeros in the complex plane actually relate to the existence and design of approximation algorithms. This connection has been well studied for general graphs, see, for example, [20, 18, 15]; for bounded-degree graphs, the picture is less clear, but the key seems to lie in understanding the underlying complex dynamical systems $[6,9,10,42,4,7]$. A general theory is so far elusive, but it seems that the chaotic behavior of the underlying complex dynamical system is linked to the presence of zeros of the partition function and to the \#P-hardness of approximation.

Establishing hardness results for ferromagnetic spin systems is notoriously challenging [32, 21]. We therefore expect our techniques to be applicable in a wider framework. We will explain in Section 2 the obstacles that arise relative to previous works for antiferromagnetic spin systems.

\subsection{Our results}

To state our inapproximability results, we first formally define the computational problems that we consider. For $z \in \mathbb{C}$, we let $|z|$ be the norm of $z, \operatorname{Arg}(z)$ be its argument in the interval $[0,2 \pi)$ and $\arg (z)=\{\operatorname{Arg}(z)+2 k \pi \mid k \in \mathbb{Z}\}$ be the set of all of its arguments. We will consider the problems of approximating the norm of the partition function $Z_{G}(\lambda, b)$ within a rational factor $K>1$ and its argument within an additive rational constant $\rho>0$. For the computational problems, we moreover assume that $b \in(0,1)$ is rational and $\lambda$ has rational real and imaginary parts. The rationality assumption is mainly for convenience (representation issues) and simplifies some of the proofs.

Name \#lsing Norm $(\lambda, b, \Delta, K)$.

Instance A graph $G=(V, E)$ with maximum degree $\leq \Delta$.

Output If $Z_{G}(\lambda, b)=0$, the algorithm may output any rational. Otherwise, it must return a rational $\widehat{N}$ such that $\widehat{N} / K \leq\left|Z_{G}(\lambda, b)\right| \leq K \widehat{N}$.

We remark here that the explicit constant $K>1$ in the problem definition above is only for convenience, having $K=2^{n^{1-\epsilon}}$ for any constant $\epsilon>0$ and with $n$ the number of vertices of the graph does not change the complexity of the problem using standard powering arguments.

Name \#lsingArg $(\lambda, b, \Delta, \rho)$.

Instance A graph $G=(V, E)$ with maximum degree $\leq \Delta$.

Output If $Z_{G}(\lambda, b)=0$, the algorithm may output any rational. Otherwise, it must return a rational $\widehat{A}$ such that $|\widehat{A}-a| \leq \rho$ for some $a \in \arg \left(Z_{G}(\lambda, b)\right)$.

A fully polynomial time approximation scheme ( FPTAS) for approximating $Z_{G}(\lambda, b)$ for given $\lambda$ and $b$ and positive integer $\Delta$ is an algorithm that for any $n$-vertex graph $G$ of maximum degree at most $\Delta$ and any rational $\varepsilon>0$ solves both probems \#Ising $\operatorname{Norm}(\lambda, b, \Delta, 1+\varepsilon)$ and \#lsingArg $(\lambda, b, \Delta, \varepsilon)$ in time polynomial in $n / \varepsilon$.

We use $\mathbb{Q}$ to denote the set of rational numbers and $\mathbb{C}_{\mathbb{Q}}$ to denote the set of complex numbers with rational real and imaginary part. We denote by $\mathbb{S}$ the unit circle in the complex plane and $\mathbb{S}_{\mathbb{Q}}=\mathbb{S} \cap \mathbb{C}_{\mathbb{Q}}$. It is well-known that numbers in $\mathbb{S}_{\mathbb{Q}}$ are dense on the unit circle. ${ }^{5}$ For $\theta \in(0, \pi)$ we denote

$$
I(\theta):=\left\{e^{i \vartheta} \mid-\theta<\vartheta<\theta\right\} .
$$

For $\Delta \geq 3$ and $b \in\left(\frac{\Delta-2}{\Delta}, 1\right)$ we denote by $\theta_{b} \in(0, \pi)$ the angle from [42, Theorem A] for which the following hold:

${ }^{5}$ See, for example, the upcoming Lemma 35. 
(i) For any graph $G$ of maximum degree at most $\Delta$ and any $\lambda \in I\left(\theta_{b}\right), Z_{G}(\lambda, b) \neq 0$.

(ii) For each $\lambda \in \mathbb{S} \backslash I\left(\theta_{b}\right)$ there exists $\lambda^{\prime} \in \mathbb{S}$ arbitrarily close to $\lambda$ and a tree $T$ of maximum degree $\Delta$ for which $Z_{T}\left(\lambda^{\prime}, b\right)=0$.

Our main result is as follows.

Theorem 1. Let $\Delta \geq 3$ be an integer and let $K=1.001$ and $\rho=\pi / 40$.

(a) Let $b \in\left(0, \frac{\Delta-2}{\Delta}\right]$ be a rational and $\lambda \in \mathbb{S}_{\mathbb{Q}}$ such that $\lambda \neq \pm 1$. Then the problems $\#$ Ising $\operatorname{Norm}(\lambda, b, \Delta, K)$ and \#Ising $\operatorname{Arg}(\lambda, b, \Delta, \rho)$ are \#P-hard.

(b) Let $b \in\left(\frac{\Delta-2}{\Delta}, 1\right)$ be a rational. Then the collection of complex numbers $\lambda \in \mathbb{S}_{\mathbb{Q}}$ for which $\#$ \#sing Norm $(\lambda, b, \Delta, K)$ and \#Ising $\operatorname{Arg}(\lambda, b, \Delta, \rho)$ are \#P-hard is dense in the arc $\mathbb{S} \backslash I\left(\theta_{b}\right)$.

Combined with [35], part (a) of our main theorem completely classifies the hardness of approximating the partition function $Z_{G}(\lambda, b)$ (as per the two computational problems stated above), for $b \in\left(0, \frac{\Delta-2}{\Delta}\right]$. Combined with [42, Corollary 1], part (b) of our main theorem 'essentially' classifies the hardness of approximating the partition function for $b \in\left(\frac{\Delta-2}{\Delta}, 1\right)$ and answers a question from [42]. Technically, we do not rule out that there may be an efficient algorithm for these problems for some $\lambda \in \mathbb{S}_{\mathbb{Q}} \backslash I\left(\theta_{b}\right)$, but such an algorithm must be specifically tailored to such a particular $\lambda$ (unless, of course, $\mathrm{P}=\# \mathrm{P}$ ). We in fact conjecture that, when $b \in\left(\frac{\Delta-2}{\Delta}, 1\right)$, approximating the partition function (as in Theorem 1) is \#P-hard for all non-real $\lambda \in \mathbb{S}_{\mathbb{Q}} \backslash I\left(\theta_{b}\right)$. See Remark 2 for a discussion of the antipodal case $\lambda=-1$.

We should further remark that the open interval $b \in\left(0, \frac{\Delta-2}{\Delta}\right)$ for positive $\lambda$ corresponds to the socalled nonuniqueness region of the infinite $\Delta$-regular tree. For the antiferromagnetic Ising model and positive $\lambda$, nonuniqueness leads to computational intractability [46, 17], in contrast to the ferromagnetic case. As we explain in Section 2, the phenomenon which underpins our proofs for $|\lambda|=1$ with $\lambda \neq \pm 1$ is the chaotic behaviour of the underlying complex dynamical system, which resembles in rough terms a complex-plane analogue of nonuniqueness. Interestingly, at criticality - that is, when $b=\frac{\Delta-2}{\Delta}-$ while the model is in uniqueness for $\lambda=1$, the chaotic behaviour is nevertheless present for non-real $\lambda$ and we show \#P-hardness for this case, too.

Remark 2. We further discuss the real cases $\lambda= \pm 1$ which are not explicitly covered by Theorem 1 . The case $\lambda=1$ admits an FPRAS [26, 22, 11], but the existence of a deterministic approximation scheme is open. We study the case $\lambda=-1$ in more detail in Section 9, where we show that the problem is not \#P-hard (assuming \#P $\neq N \mathrm{NP}$ ): using the 'high-temperature' expansion of the model, we show an oddsubgraphs formulation of the partition function (Lemma 39), which is then used to conclude (Theorem 40) that the sign of the partition function can be determined trivially, while the problem of approximating the norm of the partition function for all $\Delta \geq 3$ is equivalent to the problem of approximately counting the number of perfect matchings (even on unbounded-degree graphs); the complexity of the latter is an open problem in general, but it can be approximated with an NP-oracle [27], therefore precluding \#P-hardness.

In the next section, we give an outline of the key pieces to obtain our inapproximability results; the details of these pieces will be provided in the forthcoming sections (see also Subsection 2.5 for the organisation of the article).

\section{Proof outline}

Let $\Delta \geq 3$ be an integer, $b \in\left(0, \frac{\Delta-2}{\Delta}\right]$ and $\lambda \in \mathbb{S}_{\mathbb{Q}}$ with $\lambda \neq \pm 1$. It will be convenient to work sometimes with $d=\Delta-1$. For $z_{1}, z_{2} \in \mathbb{S}$, let $\operatorname{Arc}\left[z_{1}, z_{2}\right]$ and $\operatorname{Arc}\left(z_{1}, z_{2}\right)$ denote the counterclockwise arc in $\mathbb{S}$ from $z_{1}$ to $z_{2}$ including and excluding the endpoints, respectively. For an $\operatorname{arc} A$ on the unit circle $\mathbb{S}$, we let $\ell(A)$ denote the length of $A$. We use $\bar{z}$ to denote the conjugate of $z$. 


\subsection{Rooted-tree gadgets and complex dynamical systems}

Our reductions are based on gadgets that are rooted trees, whose analysis will be based on understanding the dynamical behaviour of certain complex maps on the unit circle, given by ${ }^{6}$

$$
f_{\lambda, k}: z \mapsto \lambda \cdot\left(\frac{z+b}{b z+1}\right)^{k}, \text { for integers } k=1, \ldots, d .
$$

We will sometimes drop $\lambda$ when it is clear from the context. To connect these maps with rooted-tree gadgets, for a graph $G=(V, E)$ and a vertex $u$ of $G$, we let $Z_{G,+u}$ be the contribution to the partition function from configurations with $\sigma(u)=+$; that is,

$$
Z_{G,+u}(\lambda, b):=\sum_{\sigma: V \rightarrow\{+,-\} ; \sigma(u)=+} \lambda^{\left|n_{+}(\sigma)\right|} b^{\delta(\sigma)},
$$

and we define analogously $Z_{G,-u}$.

Definition 3. Let $\lambda, b$ be arbitrary numbers and $T$ be a tree rooted at $r$. We say that $T$ implements the field $\lambda^{\prime}$ if $Z_{T,-r}(\lambda, b) \neq 0$ and $\lambda^{\prime}=\frac{Z_{T,+r}(\lambda, b)}{Z_{T,-r}(\lambda, b)}$. We call $\lambda^{\prime}$ the field of $T$.

The next lemma explains the relevance of the maps $f_{\lambda, 1}, \ldots, f_{\lambda, d}$ for implementing fields.

Lemma 4. Let $b \in(0,1)$ and $\lambda \in \mathbb{S}$. Let $T_{1}, T_{2}$ be rooted trees with roots $r_{1}, r_{2}$ and fields $\xi_{1}, \xi_{2} \in \mathbb{S}$, respectively. Then, the tree $T$ rooted at $r_{2}$ consisting of $T_{2}$ and $k$ distinct copies of $T_{1}$ which are attached to $r_{2}$ via an edge between $r_{2}$ and $r_{1}$ implements the field $\xi=f_{\xi_{2}, k}\left(\xi_{1}\right) \in \mathbb{S}$.

Proof. Omitting for convenience the arguments $\lambda, b$ from the partition functions, we have

$$
Z_{T,+r_{2}}=Z_{T_{2},+r_{2}}\left(Z_{T_{1},+r_{1}}+b Z_{T_{1},-r_{1}}\right)^{k}, \quad Z_{T,-r_{2}}=Z_{T_{2},-r_{2}}\left(b Z_{T_{1},+r_{1}}+Z_{T_{1},-r_{1}}\right)^{k} .
$$

Dividing these yields the result (note, $Z_{T_{2},-r_{2}} \neq 0$ and $\xi_{1}=\frac{Z_{T_{1},+r_{1}}}{Z_{T_{1}, r_{1}}} \in \mathbb{S}$, so $Z_{T,-r_{2}} \neq 0$ ); the fact that $\xi \in \mathbb{S}$ follows from footnote 6 .

Note, in particular, that all fields implemented by trees lie on the unit circle $\mathbb{S}$; cf. footnote (6). The following theorem, which lies at the heart of the construction of the gadgets, asserts that throughout the relevant range of the parameters we can in fact implement a field arbitrarily close to any number in $\mathbb{S}$. We use $\mathcal{T}_{d+1}$ to denote the set of all rooted trees with maximum degree $\leq d+1$ whose roots have degree $\leq d$.

Definition 5. Given $b \in(0,1)$ and $d \geq 2$, we denote by $\mathbb{S}_{\mathbb{Q}}(d, b)$ the collection of $\lambda \in \mathbb{S}_{\mathbb{Q}}$ for which the set of fields implemented by trees in $\mathcal{T}_{d+1}$, whose roots have degree 1 , is dense in $\mathbb{S}$.

Theorem 6. Let $d \geq 2$ be an integer.

(a) Let $b \in\left(0, \frac{d-1}{d+1}\right]$ be a rational. Then $\mathbb{S}_{\mathbb{Q}}(d, b)=\mathbb{S}_{\mathbb{Q}} \backslash\{ \pm 1\}$.

(b) Let $b \in\left(\frac{d-1}{d+1}, 1\right)$ be a rational. Then $\mathbb{S}_{\mathbb{Q}}(d, b)$ is dense in $\mathbb{S} \backslash I\left(\theta_{b}\right)$.

Theorem 6 (b) is in stark contrast to what happens for $\lambda \in I\left(\theta_{b}\right)$, where it is known that fields are confined in an arc around 1 [42]. We conjecture that in part (b) it is true that $\mathbb{S}_{\mathbb{Q}}(d, b)=\mathbb{S}_{\mathbb{Q}} \backslash$ $\left(I\left(\theta_{b}\right) \cup\{-1\}\right)$. Moreover, while in Theorem 6 we focus on rational $b$, which is most relevant for our computational problems, we note that for any real $b \in(0,1), \mathbb{S}_{\mathbb{Q}}(d, b)$ is dense in $\mathbb{S}$ in case (a) and dense in $\mathbb{S} \backslash I\left(\theta_{b}\right)$ in case (b). We suspect that case (a) is true when $\mathbb{S}_{\mathbb{Q}}$ is replaced by the collection of algebraic numbers on the unit circle and $b \in\left(0, \frac{d-1}{d+1}\right]$ is algebraic, but this seems to be challenging to prove.

Later, in Section 7, we bootstrap Theorem 6 to obtain fast algorithms to implement fields with arbitrarily small error; see Lemma 32 for the exact statement. Roughly, these fields are then used as 'probes' in our reductions to compute exactly the ratio $\frac{Z_{G,+v}(\lambda, b)}{Z_{G,-v}(\lambda, b)}$ for any graph $G$ and vertex $v$; we say more about this in Subsection 2.4. For now, we focus on the key Theorem 6 and the ideas behind its proof.

${ }^{6}$ Note that, for real $b$ and $\lambda \in \mathbb{S}$, if $z \in \mathbb{S}$, then $f_{\lambda, k}(z) \in \mathbb{S}$ as well. 


\subsection{Hardness via Julia-set density}

To prove Theorem 6, we will be interested in the set of values obtained by successive composition of the maps $f_{\lambda, k}$ in (1) starting from the point $z=1$; the main challenge in our setting is to prove that, for $\lambda, b$ as in Theorem 6 , these values are dense on the unit circle $\mathbb{S}$. Part (b) is relatively easy to prove, but the real challenge lies in proving part (a).

To understand the reason that this is challenging, let us consider the properties of the map $f_{\lambda, k}$ for some root degree $k \geq 1$ viewed as a dynamical system; cf. the upcoming Lemmas 8 and 10 for details. Then, for all $b \in(0,1)$ the following hold:

1. The 'well-behaved' regime: When $b \in\left(\frac{k-1}{k+1}, 1\right)$, there exists $\lambda_{k}=\lambda_{k}(b) \in \mathbb{S}$ with $\operatorname{Im} \lambda_{k}>0$ such that for all $\lambda$ in an arc around 1 given by $\operatorname{Arc}\left[\overline{\lambda_{k}}, \lambda_{k}\right]$, the iterates of the point $z=1$ under the map $f_{\lambda, k}$ converge to a value $R_{k}(\lambda) \in \mathbb{S}$. In fact, the map $f_{\lambda, k}$ has nice convergence/contracting properties in an arc around $z=1$ : the iterates of any point in $\operatorname{Arc}\left[1, R_{k}(\lambda)\right]$ converge to $R_{k}(\lambda)$.

2. The 'chaotic' regime: Instead, when $b \in\left(\frac{k-1}{k+1}, 1\right)$ and $\lambda \in \operatorname{Arc}\left(\lambda_{k}, \overline{\lambda_{k}}\right)$ or $b \in\left(0, \frac{k-1}{k+1}\right]$, all points in $\mathbb{S}$ belong to the so-called Julia set of the map; roughly, this means that the iterates under $f_{\lambda, k}$ of two distinct but arbitrarily close points in $\mathbb{S}$ will be separated by some absolute constant infinitely many times. In other words, the map $f_{\lambda, k}$ has a chaotic behaviour on $\mathbb{S}$.

For $b \in(0,1)$, we use $\Lambda_{k}(b)$ to denote the set of $\lambda \in \mathbb{S}$ where the degree- $k$ map $f_{\lambda, k}$ exhibits the behaviour in (2); see the relevant Definition 7 and Lemma 8. Based on item (1), it was shown in [42] that the iterates of the point $z=1$ under the successive composition of the maps in (1) stay 'trapped' in a small arc around 1 when $b \in\left(\frac{d-1}{d+1}, 1\right)$ and $\lambda \in \operatorname{Arc}\left(\overline{\lambda_{d}}, \lambda_{d}\right)$.

Instead, our goal is to tame the chaotic behaviour in item (2) to get density on $\mathbb{S}$ for fixed $b \in\left(0, \frac{d-1}{d+1}\right]$ and $\lambda \in \mathbb{S} \backslash\{ \pm 1\}$. We should emphasise here that, in the range of $b, \lambda$ we consider, the map $f_{\lambda, d}$ has the chaotic behaviour described in item (2) throughout $\mathbb{S}$, so by default it is hopeless to aim for any fine analytical understanding, and this is the major technical obstacle we need to address.

An analogous setting has been previously considered in [6], in the context of approximating the independent set polynomial. The bottleneck of showing the desired density is to first argue density around a point $x^{*}$ in the Julia set of the degree- $d$ map. Once this is done, the chaotic behaviour of the degree- $d$ map around the Julia-set point $x^{*}$ can be utilised to bootstrap the density to the whole complex plane. The key challenge here is arguing the initial density around the Julia-set point of the degree- $d$ map, since the degree- $d$ map itself is useless for creating density in the Julia set. In [6], an auxiliary Fibonacci-style recursion was used to converge to such a point $x^{*}$; the density around $x^{*}$ was then achieved by utilising the convergence to further obtain a set of contracting maps around a neighbourhood $N$ of $x^{*}$, such that the images of $N$ under the maps formed a covering of $N$.

While the contracting/covering maps framework can be adapted to our setting (see Lemma 19), the bottleneck step of obtaining the initial density around the Julia-set point requires a radically different argument: the convergence of the recursion in [6] relies on a certain linearisation property, which is not present in the case of the ferromagnetic Ising model; even worse, the recursion does not converge for all the relevant range of $b, \lambda .^{7}$

\subsection{Our approach to obtain density around a Julia-set point}

We devise a new technique to tackle the problem of showing density around a point in the Julia set of $f_{\lambda, d}$. The main idea is to exploit the chaotic behaviour of the iterates of $f_{\lambda, k}$ when $\lambda \in \Lambda_{k}(b)$ to obtain an iterate $\xi$ of 1 with an expanding derivative; that is, $\left|f_{\lambda, k}^{\prime}(\xi)\right|>1$. The existence of $\xi$ follows by general arguments from the theory of complex dynamical systems; see the relevant Lemma 15. The lower bound on the derivative is then used in careful inductive constructions to obtain families of contracting maps that cover an appropriate arc of the circle.

\footnotetext{
${ }^{7}$ In fact, determining the range of $\lambda \mathrm{s}$ where the corresponding recursion for the Ising model converges to a Julia-set point is, to the best of our knowledge, beyond known complex dynamics methods.
} 
To illustrate the main idea of this inductive construction, let us assume that the degree $d+1$ is odd. Then, using Lemma 15 and the fact that $\lambda \in \Lambda_{d}(b)$, we obtain an iterate of the point $z=1$ under the map $f_{\lambda, d}$, say $\xi$, so that $\left|f_{\lambda, d}^{\prime}(\xi)\right|>1$. The key point is to consider the map $f_{\xi, k}$ for $k=d / 2$. On one hand, if it happens that $\xi \notin \Lambda_{k}(b)$ lies in the 'well-behaved' regime of the degree- $k$ map, it can be shown that the maps $f_{\xi, k}, f_{\xi, k+1}$ are contracting/covering maps in an appropriate arc of $\mathbb{S}$, yielding the required density as needed (details of this argument can be found in Lemma 21). On the other hand, if $\xi \in \Lambda_{k}(b)$ lies in the 'chaotic' regime of the degree- $k$ map, then we can proceed inductively by finding an iterate $v$ of 1 under the map $f_{\xi, k}$ with expanding derivative $\left|f_{\xi, k}^{\prime}(v)\right|>1$ and recurse.

Technically, to carry out this inductive scheme we have to address the various integrality issues, while at the same time being careful to maintain the degrees of the trees bounded by $\Delta$. More important, we need to consider pairs/triples/quadruples of maps to ensure the contraction/covering property in the inductive step; to achieve this, we need to understand the dependence of the derivative at the fixpoint of the $k$-ary map with $k$. Here, things turn out to be surprisingly pleasant, since it turns out that $\left|f_{\lambda, k}^{\prime}(z)\right|$ depends linearly on the degree $k$ and is independent of $\lambda$; see item (i) of Lemma 10. This fact is exploited in the arguments of Subsection 4.2. These considerations cover almost all cases, but a few small degrees $d$ remain, which we cover by a Cantor-style construction; see Section 5.2 for details.

\subsection{The reduction}

The arguments discussed so far can be used to show that rooted trees in $\mathcal{T}_{d+1}$ implement any field $\xi$ on the unit circle $\mathbb{S}$ within arbitrarily small error $\epsilon>0$; see Lemma 32 for the form that we actually need. We now discuss in a bit more detail the high-level idea behind the final reduction argument in Section 8.

The key observation to utilise the gadgets is that for any graph $G$ and vertex $v$ with $Z_{G,-v}(\lambda, b) \neq 0$, the 'field' at a vertex $v$ satisfies $\frac{Z_{G,+v}(\lambda, b)}{Z_{G,-v}(\lambda, b)} \in \mathbb{S}$ (cf. Lemma 34), and hence we can use our rootedtree gadgets as probes to compute exactly the ratio $Q_{G, v}:=\frac{Z_{G,+v}(\lambda, b)}{Z_{G,-v}(\lambda, b)}$. The straightforward way to do this would be to attach a tree on $v$ which implements a field $x \in \mathbb{S}$ and use oracle calls to either $\#$ IsingNorm $(\lambda, b, \Delta, K)$ and \#lsingArg $(\lambda, b, \Delta, \rho)$ and look for $x=x^{*}$ that makes the partition function of the resulting graph equal to zero; from the key observation earlier, we know that such an $x^{*}$ exists, namely, $x^{*}=-1 / Q_{G, v}$ and, to determine it, we can use binary search.

This is the main idea behind the reduction, though there are a couple of caveats. First of all, there is no way to know whether the ratio $Q_{G, v}$ is well-defined; that is, whether $Z_{G,-v}(\lambda, b) \neq 0$, even using oracle calls to the approximation problems we study: $Z_{G,-v}(\lambda, b)$ is not a partition function of a graph (since $v$ 's spin is fixed), and even if we managed to cast this as a partition function, the oracles cannot detect zeros (cf. Subsection 1.1). The second caveat is that attaching the tree increases the degree of $v$ which is problematic when, for example, $G$ is $\Delta$-regular and the peeling-vertices argument does not quite work since there is no simple way to utilise the oracles after the first step.

The first point is addressed by replacing the edges of $G$ with paths of appropriate length, which has the effect of 'changing' the value of the parameter $b$ to some value $\hat{b}$ close but not equal to 1 where the partition function is zero-free (we actually need to attach to internal vertices of the paths rooted trees with fields close to $1 / \lambda$ so that the complex external field $\lambda$ is almost cancelled). Then, using oracle calls to \#Ising Norm $(\lambda, b, \Delta, K)$ or \#IsingArg $(\lambda, b, \Delta, \rho)$, our algorithm aims to determine the value of $Z_{G}(\lambda, \hat{b})$, which is a \#P-hard problem ([28], Theorem 1.1); the key point is that now we have zerofreeness of the partition function, which allows us to assert that the quantities we compute during the course of the algorithm are actually well-defined.

The second point is addressed by doing the peeling argument at the level of edges by trying to figure out, for an edge $e$ of $G$, the value of the ratio $\hat{Q}_{G, e}=\frac{Z_{G}(\lambda, \hat{b})}{Z_{G, \mid e}(\lambda, \hat{b})}$. We do this by subdividing the edge and use a field gadget on the middle vertex; this has the benefit that it does not increase the maximum degree of the graph, but certain complications arise since instead of $\hat{Q}_{G, e}$, we retrieve a slightly different ratio (see Lemma 37 in Subsection 8.2), and some extra work is required to finish off the proof of Theorem 1; see Subsection 8.3 for details. 


\subsection{Outline}

The next section details the dynamical properties of the maps $f_{\lambda, k}$ and elaborates on the inductive proof of Theorem 6, which is based on the upcoming Lemma 17. Section 4 explains in more detail the contracting/covering maps framework and how we utilise the degree/derivative interplay to cover the bulk of the cases in Lemma 17. Section 5 contains the remaining pieces needed to complete the proof of Lemma 17, which is given in Section 6. In Section 7, we bootstrap Theorem 6 to obtain fast algorithms to implement fields on the unit circle with arbitrarily small precision error, which is used in the reduction arguments of Section 8, where the proof of Theorem 1 is completed. Finally, in Section 9, we study the case $\lambda=-1$ (cf. Remark 2) and show the equivalence with the problem of approximately counting perfect matchings.

\section{Complex Dynamics Preliminaries and the Inductive Step in Theorem 6}

In this section, we set up some preliminaries about the maps $f_{\lambda, k}$ in (1) that will be used to prove Theorem 6. We first consider the general case $k \geq 1$ in Subsection 3.1 and then further study the $k=1$ case separately in Subsection 3.2. In Subsection 3.3, we use these properties to obtain points with expanding derivatives using tools from complex dynamics. Then, in Subsection 3.4, we give the main lemma that lies at the heart of the inductive proof of Theorem 6 and conclude the proof of the latter.

\subsection{Results on $f_{\lambda, k}$ for general $k$}

This section contains relevant properties of the maps $f_{\lambda, k}: z \mapsto \lambda \cdot\left(\frac{z+b}{b z+1}\right)^{k}$ that we will need; these were discussed informally in Subsection 2.2, and here we formalise them. Almost all results of this section follow from arguments in [42].

We begin by formally defining the set $\Lambda_{k}(b)$.

Definition 7. Let $k \geq 1$ be an integer and $b \in(0,1)$. We let $\Lambda_{k}(b)$ be the set of $\lambda \in \mathbb{S}$ such that all fixed points $z$ of the map $f_{\lambda, k}$ with $z \in \mathbb{S}$ are repelling; that is, $\left|f_{\lambda, k}^{\prime}(z)\right|>1$.

The following lemma gives a description of the set $\Lambda_{k}(b)$ and characterises the Julia set of $f_{\lambda, k}$. We have described informally the dynamical properties of the map $f_{\lambda, k}$ that the Julia set captures; see item (2) in Subsection 2.2. The reader is referred to [38, Chapter 4] for more details on the general theory.

Lemma 8. Let $k \geq 1$ be an integer. Then,

○ if $b \in\left(0, \frac{k-1}{k+1}\right), \Lambda_{k}(b)=\mathbb{S}$. For $b=\frac{k-1}{k+1}, \Lambda_{k}(b)=\mathbb{S} \backslash\{+1\}$.

○ if $b \in\left(\frac{k-1}{k+1}, 1\right)$, there is $\lambda_{k}=\lambda_{k}(b) \in \mathbb{S}$ with $\operatorname{Im}\left(\lambda_{k}\right)>0$ such that $\Lambda_{k}(b)=\operatorname{Arc}\left(\lambda_{k}, \overline{\lambda_{k}}\right)$.

Moreover, if $k>1$, then for all $\lambda \in \Lambda_{k}(b)$, the Julia set of $f_{\lambda, k}$ is equal to the unit circle $\mathbb{S}$.

Proof. For $b \in\left(\frac{k-1}{k+1}, 1\right)$, the range of $\Lambda_{k}(b)$ follows from [42, Theorem 14]. For $b \in\left(0, \frac{k-1}{k+1}\right]$, the range of $\Lambda_{k}(b)$ follows from item (i) in Lemma 10. The characterisation of the Julia set for $\lambda \in \Lambda_{k}(b)$ is shown in [42, Proof of Proposition 17].

Remark 9. The $\lambda_{k}(b)$ of the lemma is equal to $e^{i \theta_{b}}$ from the statement of Theorem 1 (where we replace $\Delta$ by $k+1)$.

Let $A$ be an arc of $\mathbb{S}$. A map $f: A \rightarrow \mathbb{S}$ is orientation-preserving if for any $z, z_{1}, z_{2} \in A$ with $z \in \operatorname{Arc}\left[z_{1}, z_{2}\right]$ it holds that $f(z) \in \operatorname{Arc}\left[f\left(z_{1}\right), f\left(z_{2}\right)\right]$. The orbit of a point $z_{0} \in \mathbb{S}$ under the map $f_{\lambda, k}$ is the sequence of the iterates $\left\{f_{\lambda, k}^{n}\left(z_{0}\right)\right\}_{n \geq 0}$. A fixed point $z$ of the map $f_{\lambda, k}$ is called attracting if $\left|f_{\lambda, k}^{\prime}(z)\right|<1$ and parabolic if $\left|f_{\lambda, k}^{\prime}(z)\right|=1$.

The following lemma captures properties of the maps $f_{\lambda, k}$ when $k \in\{1, \ldots, d-1\}$ in the regime $b \in\left(\frac{d-2}{d}, \frac{d-1}{d+1}\right]$, which turns out to be the hard part of the proof of Theorem 6 (the lemma is stated more generally for $\left.b \in\left(\frac{d-2}{d}, 1\right)\right)$. 
Lemma 10. Let $d \in \mathbb{Z}_{\geq 2}$ and let $b \in\left(\frac{d-2}{d}, 1\right)$. Then the following hold:

(i) For all $\lambda \in \mathbb{S}$ and $k \in \mathbb{Z}_{\geq 1}$, the map $f_{\lambda, k}: \mathbb{S} \rightarrow \mathbb{S}$ is orientation-preserving. Also, the magnitude of the derivative at a point $z \in \mathbb{S}$ does not depend on $\lambda$ and equals $\left|f_{k}^{\prime}(z)\right|$, where

$$
\left|f_{k}^{\prime}(z)\right|=k \cdot\left|f_{1}^{\prime}(z)\right|=\frac{k\left(1-b^{2}\right)}{b^{2}+2 b \cdot \operatorname{Re}(z)+1} .
$$

(ii) For $k \in\{1, \ldots, d-1\}$, let $\lambda_{k}=\lambda_{k}(b) \in \mathbb{S}$ be as in Lemma 8. Then,

$\circ$ if $\lambda \in \operatorname{Arc}\left(\overline{\lambda_{k}}, \lambda_{k}\right)$, then $f_{\lambda, k}$ has a unique attracting fixed point $R_{k}(\lambda) \in \mathbb{S}$.

○ if $\lambda=\overline{\lambda_{k}}$ or $\lambda_{k}$, then $f_{\lambda, k}$ has a unique parabolic fixed point $R_{k}(\lambda) \in \mathbb{S}$.

(iii) The fixed point maps $R_{k}: \operatorname{Arc}\left[\overline{\lambda_{k}}, \lambda_{k}\right] \rightarrow \mathbb{S}$ are continuously differentiable on $\operatorname{Arc}\left(\overline{\lambda_{k}}, \lambda_{k}\right)$ and orientation-preserving with the property that $R_{k}(1)=1$ and $R_{k}(\bar{\lambda})=\overline{R_{k}(\lambda)}$.

(iv) For $\lambda \in \operatorname{Arc}\left(1, \lambda_{k}\right]$, the fixed point $R_{k}(\lambda)$ is in the upper half-plane. For $z_{0} \in \operatorname{Arc}\left[1, R_{k}(\lambda)\right]$, the orbit of $z_{0}$ under iteration of $f_{\lambda, k}$ converges to $R_{k}(\lambda)$ and is contained in $\operatorname{Arc}\left[z_{0}, R_{k}(\lambda)\right]$.

(v) The following inequalities hold:

$$
\operatorname{Arg}\left(\lambda_{d-1}\right)<\operatorname{Arg}\left(\lambda_{d-2}\right)<\cdots<\operatorname{Arg}\left(\lambda_{1}\right),
$$

while for $\lambda \in \operatorname{Arc}\left(1, \lambda_{m}\right]$, with $m \leq d-1$, we have

$$
\operatorname{Arg}\left(R_{1}(\lambda)\right)<\operatorname{Arg}\left(R_{2}(\lambda)\right)<\cdots<\operatorname{Arg}\left(R_{m}(\lambda)\right)
$$

with the additional property that, for $i \in\{1, \ldots, m-2\}$,

$$
\ell\left(\operatorname{Arc}\left[R_{i}(\lambda), R_{i+1}(\lambda)\right]\right) \leq \ell\left(\operatorname{Arc}\left[R_{i+1}(\lambda), R_{i+2}(\lambda)\right]\right) .
$$

Proof. We refer to [42] for proofs of items (i)-(iv). Specifically, item (i) follows from [42, Lemma 8 \& Equation (3.1)], item (ii) from [42, Lemma 13, Theorem 14, Proof of Proposition 17], item (iii) from [42, Proof of Theorem 14] and item (iv) from [42, Theorem 14, Proof of Theorem 5(i)].

We will prove item (v). By taking the derivative of both sides of the equality $f_{\lambda, k}\left(R_{k}(\lambda)\right)=R_{k}(\lambda)$ with respect to $\lambda$ and rewriting, we obtain

$$
R_{k}^{\prime}(\lambda)=\frac{R_{k}(\lambda)}{\lambda\left(1-f_{\lambda, k}^{\prime}\left(R_{k}(\lambda)\right)\right)} .
$$

Using equation (2) for $z=R_{m}(1)=1$, we obtain that $R_{i+1}^{\prime}(1)>R_{i}^{\prime}(1)$ for $i \in\{1, \ldots, d-2\}$, and thus for $\lambda \in \mathbb{S}$ in the upper half-plane near 1 we find that $\operatorname{Arg}\left(R_{i+1}(\lambda)\right)>\operatorname{Arg}\left(R_{i}(\lambda)\right)$.

The derivative at a fixed point of a map of the unit circle to itself is real (see also [42, Lemma 11]). Furthermore, if such a map is orientation-preserving, the derivative at a fixed point is positive. Because the map $f_{\lambda, i}$ is orientation-preserving with attracting fixed point $R_{i}(\lambda)$, we find that $f_{\lambda, i}^{\prime}\left(R_{i}(\lambda)\right) \in(0,1)$ for $\lambda \in \operatorname{Arc}\left(\overline{\lambda_{i}}, \lambda_{i}\right)$. From this, we deduce that we can write

$$
\left|R_{k}^{\prime}(\lambda)\right|=\frac{1}{1-f_{\lambda, k}^{\prime}\left(R_{k}(\lambda)\right)} .
$$

From equation (2) it can be seen that $\left|f_{i}^{\prime}(z)\right|$ is increasing both with respect to $\operatorname{Arg}(z)$ when $\operatorname{Im}(z)>0$ and with respect to the index $i$ and thus, as long as $R_{i}(\lambda)$ and $R_{i+1}(\lambda)$ are both defined and $\operatorname{Arg}\left(R_{i+1}(\lambda)\right)>$ $\operatorname{Arg}\left(R_{i}(\lambda)\right)$, we deduce that $\left|R_{i+1}^{\prime}(\lambda)\right|>\left|R_{i}^{\prime}(\lambda)\right|$. Since $\operatorname{Arg}\left(R_{i+1}(\lambda)\right)>\operatorname{Arg}\left(R_{i}(\lambda)\right)$ for $\lambda$ in the upper half-plane close to 1 , we conclude that there cannot be any $\lambda$ in the upper half-plane such that $\operatorname{Arg}\left(R_{i+1}(\lambda)\right) \leq \operatorname{Arg}\left(R_{i}(\lambda)\right)$.

Now suppose that there is some index $i$ such that $\operatorname{Arg}\left(\lambda_{i}\right) \leq \operatorname{Arg}\left(\lambda_{i+1}\right)$. Note that $R_{i}\left(\lambda_{i}\right)$ is a parabolic fixed point of $f_{\lambda_{i}, i}$, which means that $f_{\lambda_{i}, i}^{\prime}\left(R_{i}\left(\lambda_{i}\right)\right)=1$. Because we assumed that $\operatorname{Arg}\left(\lambda_{i}\right) \leq \operatorname{Arg}\left(\lambda_{i+1}\right)$, 
we see from item (ii) that $R_{i+1}\left(\lambda_{i}\right)$ must be well-defined. We already deduced that $\operatorname{Arg}\left(R_{i+1}\left(\lambda_{i}\right)\right)>$ $\operatorname{Arg}\left(R_{i}\left(\lambda_{i}\right)\right)$ and thus $f_{\lambda_{i}, i+1}^{\prime}\left(R_{i+1}\left(\lambda_{i}\right)\right)>f_{\lambda_{i}, i}^{\prime}\left(R_{i}\left(\lambda_{i}\right)\right)=1$, which contradicts the fact that $R_{i+1}\left(\lambda_{i}\right)$ is an attracting fixed point of $f_{\lambda_{i}, i+1}$. This concludes the proof of the first two claims of item (v).

Finally, we show the final claim of item (v). For indices $0 \leq i \leq j \leq m$, it will be convenient to denote by $A_{i, j, \lambda}$ the $\operatorname{arc} \operatorname{Arc}\left[R_{i}(\lambda), R_{j}(\lambda)\right]$, under the convention that $R_{0}(\lambda)=1$. For $\lambda \in \operatorname{Arc}\left(1, \lambda_{m}\right]$, our goal is hence to show that $\ell\left(A_{i, i+1, \lambda}\right) \leq \ell\left(A_{i+1, i+2, \lambda}\right)$ for all $i \in\{1, \ldots, m-2\}$.

For any $\lambda \in \operatorname{Arc}\left(1, \lambda_{k}\right]$, we observe for $i=1, \ldots, k$ that

$$
\ell\left(A_{0, i, \lambda}\right)=\int_{\operatorname{Arc}[1, \lambda]}\left|R_{i}^{\prime}(z)\right||d z|
$$

and thus for $i \in\{1, \ldots, k-1\}$ we have $\ell\left(A_{i, i+1, \lambda}\right)=\int_{\operatorname{Arc}[1, \lambda]}\left|R_{i+1}^{\prime}(z)\right|-\left|R_{i}^{\prime}(z)\right||d z|$.

We first show item (v) for $\lambda$ near 1 . As we let $\lambda$ approach 1 along the circle, we obtain that

$$
\begin{aligned}
\lim _{\lambda \rightarrow 1} \frac{\ell\left(A_{i, i+1, \lambda}\right)}{\ell(\operatorname{Arc}[1, \lambda])} & =\left|R_{i+1}^{\prime}(1)\right|-\left|R_{i}^{\prime}(1)\right|=\frac{1}{1-f_{\lambda, i+1}^{\prime}(1)}-\frac{1}{1-f_{\lambda, i}^{\prime}(1)} \\
& =\frac{(1+b) /(1-b)}{(i-(1+b) /(1-b))(i-2 b /(1-b))} .
\end{aligned}
$$

The second equality can be obtained by using (4) and the third by using (2) and simplifying. If we denote this expression by $g(i)$, then it is not hard to see that $g(i+1)>g(i)$ as long as $i+1<2 b /(1-b)$. Because $b \in\left(\frac{d-2}{d}, 1\right)$, we have $2 b /(1-b)>d-2$. So, indeed, $g(i+1)>g(i)$ for $i \in\{1, \ldots, d-3\}$, which contains $\{1, \ldots, k-2\}$. This shows that the inequality in (3) is true for $\lambda$ near 1 .

Now suppose that there is $\lambda \in \mathbb{S}$ and index $i$ for which the inequality in (3) does not hold. Then, by continuity, because the inequality does hold near 1 , there is a $\lambda \in \mathbb{S}$ for which the inequality is an equality; that is,

$$
\ell\left(A_{i, i+1, \lambda}\right)=\ell\left(A_{i+1, i+2, \lambda}\right)
$$

For convenience, we will henceforth drop the subscript $\lambda$ from the notation for the $\operatorname{arcs} A_{i, j, \lambda}$ and simply write $A_{i, j}$. The maps $f_{\lambda, j}$ are orientation-preserving for any $j$ and thus

$$
\ell\left(\operatorname{Arc}\left[\lambda, R_{j}(\lambda)\right]\right)=\ell\left(f_{\lambda, j}\left(A_{0, j}\right)\right)=\int_{A_{0, j}}\left|f_{j}^{\prime}(z)\right||d z| .
$$

Using this equality we can write

$$
\ell\left(A_{j, j+1}\right)=\int_{A_{0, j+1}}\left|f_{j+1}^{\prime}(z)\right||d z|-\int_{A_{0, j}}\left|f_{j}^{\prime}(z)\right||d z| .
$$

We use this equality for $j=i$ and $j=i+1$ and rearrange (5) to obtain

$$
2 \int_{A_{0, i+1}}\left|f_{i+1}^{\prime}(z)\right||d z|=\int_{A_{0, i+2}}\left|f_{i+2}^{\prime}(z)\right||d z|+\int_{A_{0, i}}\left|f_{i}^{\prime}(z)\right||d z| .
$$

We use (2) to rewrite the left-hand side of this equation as

$$
2(i+1) \int_{A_{0, i}}\left|f_{1}^{\prime}(z)\right||d z|+2 \int_{A_{i, i+1}}\left|f_{i+1}^{\prime}(z)\right||d z|
$$


and we rewrite the right-hand side as

$$
(i+2) \int_{A_{0, i}}\left|f_{1}^{\prime}(z)\right||d z|+\int_{A_{i, i+2}}\left|f_{i+2}^{\prime}(z)\right||d z|+i \cdot \int_{A_{0, i}}\left|f_{1}^{\prime}(z)\right||d z| .
$$

These two being equal implies that

$$
2 \int_{A_{i, i+1}}\left|f_{i+1}^{\prime}(z)\right||d z|=\int_{A_{i, i+2}}\left|f_{i+2}^{\prime}(z)\right||d z| .
$$

We will show that this yields a contradiction. We rewrite the right-hand side as

$$
\int_{A_{i, i+2}}\left|f_{i+2}^{\prime}(z)\right||d z|=\int_{A_{i, i+1}}\left|f_{i+2}^{\prime}(z)\right||d z|+\int_{A_{i+1, i+2}}\left|f_{i+2}^{\prime}(z)\right||d z|,
$$

and we will show that both summands are greater than $\int_{A_{i, i+1}}\left|f_{i+1}^{\prime}(z)\right||d z|$, which will yield the contradiction. The inequality for the first summand follows easily from the fact that $\left|f_{i+2}^{\prime}(z)\right|>\left|f_{i+1}^{\prime}(z)\right|$ for all $z \in \mathbb{S}$; cf. (2). The second inequality uses the fact that $\left|f_{i+2}^{\prime}(z)\right|$ increases as $\operatorname{Arg}(z)$ increases (when $\operatorname{Im} z>0)$ and thus

$$
\begin{aligned}
\int_{A_{i+1, i+2}}\left|f_{i+2}^{\prime}(z)\right||d z| & >\left|f_{i+2}^{\prime}\left(R_{i+1}(\lambda)\right)\right| \cdot \ell\left(A_{i+1, i+2}\right)>\left|f_{i+1}^{\prime}\left(R_{i+1}(\lambda)\right)\right| \cdot \ell\left(A_{i, i+1}\right) \\
& >\int_{A_{i, i+1}}\left|f_{i+1}^{\prime}(z)\right||d z| .
\end{aligned}
$$

The second inequality of this derivation uses the assumed equality in (5). This yields the desired contradiction.

Remark 11. For any $\lambda \in \mathbb{S}$ and $k \in \mathbb{Z}_{\geq 1}$ for which the fixed point $R_{k}(\lambda)$ is defined, we have $\left|f_{k}^{\prime}\left(R_{k}(\lambda)\right)\right|>\left|f_{k}^{\prime}(\lambda)\right|$. To see this when $\operatorname{Im} \lambda>0$, note from item (iv) and the fact that the maps $f_{\lambda, k}$ are orientation-preserving that $\operatorname{Arg}\left(R_{k}(\lambda)\right) \in(\operatorname{Arg}(\lambda), \pi)$ and hence by (2) that $\left|f_{k}^{\prime}\left(R_{k}(\lambda)\right)\right|>\left|f_{k}^{\prime}(\lambda)\right|$. When $\operatorname{Im} \lambda<0$, the inequality follows from the above since $\overline{R_{k}(\lambda)}=R_{k}(\bar{\lambda})$ from item (iii) and the expression in (2) depends only the real part of $z$.

\subsection{Results on $f_{\lambda, 1}$}

Note that $f_{\lambda, 1}$ is a Möbius transformation; we will extract some relevant information about it using the theory of Möbius transformations, following [3, Section 4.3].

There is a natural way to relate each Möbius transformation $g$ with a $2 \times 2$ matrix $A$. Formally, let $\mathrm{GL}_{2}(\mathbb{C})$ be the group of $2 \times 2$ invertible matrices with complex entries (with the multiplication operation) and $\mathcal{M}$ be the group of Möbius transformations (with the composition operation $\circ$ ). The following map gives a surjective homomorphism between the groups $\mathrm{GL}_{2}(\mathbb{C})$ and $\mathcal{M}$ :

$$
\Phi: \mathrm{GL}_{2}(\mathbb{C}) \rightarrow \mathcal{M}, \quad\left(\begin{array}{ll}
a & b \\
c & d
\end{array}\right) \mapsto\left(z \mapsto \frac{a z+b}{c z+d}\right)
$$

For $g \in \mathcal{M}$, let $A \in \mathrm{GL}_{2}(\mathbb{C})$ such that $\Phi(A)=g$ and define $\operatorname{tr}^{2}(g)=\operatorname{tr}(A)^{2} / \operatorname{det}(A)$. This value does not depend on the choice of preimage and, thus, $\operatorname{tr}^{2}$ is a well-defined operator on $\mathcal{M}$. In the following theorem, it is stated how this operator is used to classify Möbius transformations. We say that $f, g \in \mathcal{M}$ are conjugate if there is $h \in \mathcal{M}$ such that $f=h \circ g \circ h^{-1}$. 
Theorem 12 ([3, Theorem 4.3.4]). Let $g \in \mathcal{M}$ not equal to the identity; then $g$ is conjugate to

1. a rotation $z \mapsto e^{i \theta} z$ for some $\theta \in(0, \pi]$ if and only if $\operatorname{tr}^{2}(g) \in[0,4)$, in which case $\operatorname{tr}^{2}(g)=$ $2 \cdot(\cos (\theta)+1)$

2. a multiplication $z \mapsto e^{\theta} z$ for some $\theta \in \mathbb{R}_{>0}$ if and only if $\operatorname{tr}^{2}(g) \in(4, \infty)$, in which case $\operatorname{tr}^{2}(g)=$ $2 \cdot(\cosh (\theta)+1)$.

In case (1), $g$ is said to be elliptic, while in case (2) $g$ is called hyperbolic. $\operatorname{If}^{2} \operatorname{tr}^{2}(g)=4$ the map is called parabolic.

Corollary 13. Let $b \in(0,1)$, and let $\lambda_{1}=\lambda_{1}(b) \in \mathbb{S}$ be as in Lemma 8. The map $f_{\lambda, 1}$ is hyperbolic when $\lambda \in \operatorname{Arc}\left(\overline{\lambda_{1}}, \lambda_{1}\right)$, and $f_{\lambda, 1}$ is elliptic when $\lambda \in \operatorname{Arc}\left(\lambda_{1}, \overline{\lambda_{1}}\right)$.

Proof. Write $\lambda=x+i y$ with $x, y \in \mathbb{R}$ such that $x^{2}+y^{2}=1$. A short calculation gives that

$$
\operatorname{tr}^{2}\left(f_{\lambda, 1}\right)=\frac{2(x+1)}{1-b^{2}}
$$

The value of $t^{2}\left(f_{\lambda, 1}\right)$ strictly increases from 0 to $4 /\left(1-b^{2}\right)$ as $x$ increases from -1 to 1 . It follows that there is a unique value $x \in(-1,1)$ such that $\operatorname{tr}^{2}\left(f_{\lambda, 1}\right)=4$. This value must coincide with $\operatorname{Re}\left(\lambda_{1}\right)$, where $\lambda_{1}=\lambda_{1}(b) \in \mathbb{S}$ is as in Lemma 8 , completing the proof.

Lemma 14. Let $b \in(0,1)$ be a rational. Suppose that $\xi \in \mathbb{S}_{\mathbb{Q}}$ with $\xi \neq \pm 1$ is such that $f_{\xi, 1}$ is elliptic. Then $f_{\xi, 1}$ is conjugate to an irrational rotation.

Proof. Let $\xi=x+i y$ with $x, y \in \mathbb{Q}$ such that $x^{2}+y^{2}=1$. Because $f_{\xi, 1}$ is elliptic, it is conjugate to a rotation $z \mapsto e^{i \theta} z$ with

$$
2 \cdot(\cos (\theta)+1)=\frac{2(x+1)}{1-b^{2}} .
$$

Let $t=2 \cdot(\cos (\theta)+1)$. Suppose $\theta$ is an angle corresponding to a rational rotation; that is, if we let $z=e^{i \theta}$, then there is a natural number $n$ such that $z^{n}=1$. It follows that then $\bar{z}^{n}=1$ and thus both $z$ and $\bar{z}$ are also algebraic integers. Therefore, $z+\bar{z}=2 \cos (\theta)$ is an algebraic integer. It follows that $t$ is an algebraic integer, while the right-hand side of (6) shows that $t$ must also be rational. Because the only rational algebraic integers are integers, we can conclude that $t$ must be an integer and thus $t \in\{0,1,2,3\}$. If $t=0$, we see that $\xi=x=-1$, which we excluded, so only three possible values of $t$ remain. Let $X=t(1+b) /(1-b)$ and $Y=2 t y /(1-b)^{2}$; then $(X, Y)$ is a rational point on the elliptic curve $E_{t}$ given by the following equation:

$$
E_{t}: Y^{2}=X^{3}-(t-2) t \cdot X^{2}+t^{2} \cdot X .
$$

The set of rational points of an elliptic curve together with an additional point has a group structure that is isomorphic to $\mathbb{Z}^{r} \times \mathbb{Z} / N \mathbb{Z}$. The number $r \geq 0$ is called the rank of the curve and the subgroup isomorphic to $\mathbb{Z} / N \mathbb{Z}$ is called the torsion subgroup. The rank and the torsion subgroup of a particular curve can be found using a computer algebra system. Using Sage, if the variable $t$ is declared to be either 1,2 or 3, the curve $E_{t}$ can be defined with the code $E t=\operatorname{EllipticCurve}([\theta,-(\mathrm{t}-2) * \mathrm{t}, 0, \mathrm{t} * 2,0])$. The rank and the torsion subgroup can subsequently be found with the commands Et.rank() and Et.torsion_subgroup(). We find that $E_{t}$ has rank 0 for all $t \in\{1,2,3\}$. For $t \in\{1,3\}$ the torsion subgroup is isomorphic to $\mathbb{Z} / 2 \mathbb{Z}$, and for $t=2$ it is isomorphic to $\mathbb{Z} / 4 \mathbb{Z}$. This means that there is one rational point on $E_{1}$ and $E_{3}$, which we can see is the point $(0,0)$, and there are three rational points on $E_{2}$, namely, $\{(0,0),(2, \pm 4)\}$. These points do not correspond to values of $b$ within the interval $(0,1)$, which means that $\theta$ cannot correspond to a rational rotation. 


\subsection{Obtaining points with expanding derivatives}

In this section, we use the dynamical study of the maps $f_{\lambda, k}$ from previous sections to conclude the existence of points with expanding derivatives. More precisely, we show the following.

Lemma 15. Let $b \in(0,1), k \geq 1$ be an integer, and $\xi \in \Lambda_{k}(b)$ with $\xi \neq-1$. Let $z_{0} \in \mathbb{S}$ and let $z_{n}=f_{\xi, k}^{n}\left(z_{0}\right)$ for $n>0$. Then there is some index $m$ such that $\left|f_{\xi, k}^{\prime}\left(z_{m}\right)\right|>1$.

Proof. For $k=1$ it follows from Corollary 13 that $f_{\xi, 1}$ is conjugate to a rotation. If $f_{\xi, 1}$ is conjugate to an irrational rotation, then the orbit of any initial point $z_{0}$ will get arbitrarily close to -1 for which $\left|f_{1}^{\prime}(-1)\right|=\frac{1+b}{1-b}>1$. Otherwise, if $f_{\xi, 1}$ is conjugate to a rational rotation, there is an integer $N>1$ such that $f_{\xi, 1}^{N}(z)=z$ for all $z$; consider the smallest such integer $N$. Let $\theta \in(0, \pi]$ be the angle such that $f_{\xi, 1}$ is conjugate to the rotation $z \mapsto e^{i \theta} \cdot z$. Equation (6) then states that

$$
2 \cdot(\cos (\theta)+1)=\frac{2(\operatorname{Re}(\xi)+1)}{1-b^{2}} .
$$

If $N=2$, then $\theta=\pi$ and thus $\operatorname{Re}(\xi)=-1$, contradicting $\xi \neq-1$. Hence, $N>2$. From $f_{\xi, 1}^{N}(z)=z$, we obtain

$$
\prod_{n=0}^{N-1} f_{\xi, 1}^{\prime}\left(z_{n}\right)=\left(f_{\xi, 1}^{N}\right)^{\prime}\left(z_{0}\right)=1
$$

From (2), there are precisely two values of $w \in \mathbb{S}$ such that $\left|f_{\xi, 1}^{\prime}(w)\right|=1$. Because $N>2$ and $N$ is the smallest integer such that $f_{\xi, 1}^{N}\left(z_{0}\right)=z_{0}$, we conclude there is at least one term, say with index $m$, of the product in (7) for which $\left|f_{\xi, 1}^{\prime}\left(z_{m}\right)\right|>1$.

Consider now the case $k \geq 2$ and denote $f=f_{\xi, k}$. By Lemma 8 , for $\xi \in \Lambda_{k}(b)$ the Julia set of $f$ is the circle $\mathbb{S}$. In [42, Proof of Proposition 17], it is shown that the two Fatou components of $f$, denoted by $\mathbb{D}$ and $\overline{\mathbb{D}}^{c}$, are attracting basins and contain the critical points $-b$ and $-1 / b$. From $[38$, Theorem 19.1], we therefore conclude that the map $f$ is hyperbolic; that is, there exists a conformal metric $\mu$ on a neighbourhood $U$ of $\mathbb{S}$ such that $\left\|D f_{z}\right\|_{\mu} \geq \kappa>1$ for a constant $\kappa$ and all $z \in \mathbb{S}$. We will briefly expand on the meaning of this notation.

For any $z \in U$ the metric $\mu$ induces a norm $\|\cdot\|_{\mu}$ on the tangent space of $U$ at $z$ denoted by $T U_{z}$. For $z \in \mathbb{S}$ the map $f$ induces a linear map, the derivative of $f$ at $z, D f_{z}: T U_{z} \rightarrow T U_{f(z)}$. For nonzero $v \in D f_{z}$, the ratio $\left\|D f_{z}(v)\right\|_{\mu} /\|v\|_{\mu}$ is independent of the choice $v$ and is denoted by $\left\|D f_{z}\right\|_{\mu}$, the norm of $D f_{z}$.

Because $\mathbb{S}$ is compact and the metric $\mu$ is conformal, there is a constant $c>0$ such that $\left|g^{\prime}(z)\right|>$ $c \cdot\left\|D g_{z}\right\|_{\mu}$ for all $z \in \mathbb{S}$ and maps $g: \mathbb{S} \rightarrow \mathbb{S}$. It follows that for all $N>0$,

$$
\prod_{n=0}^{N-1}\left|f^{\prime}\left(z_{n}\right)\right|=\left|\left(f^{N}\right)^{\prime}\left(z_{0}\right)\right|>c \cdot\left\|D f_{z_{0}}^{N}\right\|_{\mu} \geq c \cdot \kappa^{N} .
$$

There is an $N>0$ such that the right-hand side of this equation is greater than 1 . The product on the lefthand side of the equation shows that for such an $N$ there must be at least one index $m \in\{0, \ldots, N-1\}$ such that $\left|f^{\prime}\left(z_{m}\right)\right|>1$.

Lemma 16. Let $b \in(0,1), \lambda \in \mathbb{S} \backslash\{ \pm 1\}$ and $d, k \in \mathbb{Z}_{\geq 1}$. Supppose there exists a rooted tree in $\mathcal{T}_{d+1}$ whose root degree $m$ is at most $d-k$ and which implements a field $\xi \in \Lambda_{k}(b) \backslash\{-1\}$.

Then there is $\sigma \in \mathbb{S}$ with $\left|f_{k}^{\prime}(\sigma)\right|>1$ and a sequence of rooted trees $\left\{T_{n}\right\}_{n \geq 1}$ in $\mathcal{T}_{d+1}$ with root degrees at most $m+k$ which implement a sequence of fields $\left\{\zeta_{n}\right\}_{n \geq 1}$ such that $\zeta_{n}$ approaches $\sigma$ without being equal to $\sigma$. 
Proof. Consider the orbit

$$
\mathcal{S}=\left\{f_{\lambda, 1}^{n}(1): n \geq 1\right\}
$$

Note that the elements of $\mathcal{S}$ are fields of paths. We have seen in Subsection 3.2 that either $f_{\lambda, 1}$ is conjugate to an irrational rotation or the orbit of 1 tends towards an attracting or a parabolic fixed point. In either case there is $\sigma_{0} \in \mathbb{S}$ such that $\sigma_{0} \notin \mathcal{S}$ and the elements of $\mathcal{S}$ accumulate on $\sigma_{0}$. It follows from Lemma 15 that there is a positive integer $N$ such that $\sigma:=f_{\xi, k}^{N}\left(\sigma_{0}\right)$ has the property $\left|f_{k}^{\prime}(\sigma)\right|>1$. Now define

$$
\mathcal{R}=\left\{f_{\xi, k}^{N}(s): s \in \mathcal{S}\right\} .
$$

By assumption, $\xi$ can be implemented by a rooted tree in $\mathcal{T}_{d+1}$ with root degree $m \leq d-k$, so by inductively applying Lemma 4 , the elements of $\mathcal{R}$ are fields of trees in $\mathcal{T}_{d+1}$ whose root degree is $m+k \leq d$. There is a sequence $\left\{\zeta_{n}\right\}_{n \geq 1} \subseteq \mathcal{R}$ accumulating on $\sigma$ without being equal to $\sigma$, which is what we wanted to show.

\subsection{The main lemma to carry out the induction: proof of Theorem 6}

We are now ready to state the following lemma, which will imply Theorem 6. In this section we will show how Theorem 6 follows from this lemma and the next couple of sections are dedicated to proving Lemma 17.

Lemma 17. Let $k, d \in \mathbb{Z}_{\geq 2}$ with $k \leq d, b \in\left(\frac{d-2}{d}, \frac{d-1}{d+1}\right] \cap \mathbb{Q}$ and $\lambda \in \mathbb{S}_{\mathbb{Q}} \backslash\{ \pm 1\}$. Suppose there exists $a$ rooted tree in $\mathcal{T}_{d+1}$ with root degree at most $d-k$ that implements a field $\xi \neq 1$ with the property that $\left|f_{k}^{\prime}(\xi)\right| \geq 1$ and $\xi \in \operatorname{Arc}\left[\overline{\lambda_{\lfloor k / 2\rfloor}}, \lambda_{\lfloor k / 2\rfloor}\right]$. Then the set offields implemented by trees in $\mathcal{T}_{d+1}$ is dense in $\mathbb{S}$.

Using this lemma, we can prove Theorem 6, which we restate here for convenience.

Theorem 6. Let $d \geq 2$ be an integer.

(a) Let $b \in\left(0, \frac{d-1}{d+1}\right]$ be a rational. Then $\mathbb{S}_{\mathbb{Q}}(d, b)=\mathbb{S}_{\mathbb{Q}} \backslash\{ \pm 1\}$.

(b) Let $b \in\left(\frac{d-1}{d+1}, 1\right)$ be a rational. Then $\mathbb{S}_{\mathbb{Q}}(d, b)$ is dense in $\mathbb{S} \backslash I\left(\theta_{b}\right)$.

Proof. We start with the proof of part (b). Let $\lambda^{\prime} \in \mathbb{S} \backslash I\left(\theta_{b}\right)$. By [42, Corollary 4 and Theorem 5], it follows that there exists $\lambda \in \mathbb{S}$ arbitrarily close to $\lambda^{\prime}$ for which there exists a tree $T \in \mathcal{T}_{d+1}$ such that $Z_{T}(\lambda, b)=0$. Choose such a tree $T$ with the minimum number of vertices and let $v$ be a leaf of $T$, from now on referred to as the root of $T$. Denote $T^{\prime}=T-v$ and let $u$ be the unique neighbour of $v$ in $T$. Then $Z_{T,-v}(\lambda, b) \neq 0$. Indeed, if $Z_{T,-v}(\lambda, b)=0$, then $Z_{T,+v}(\lambda, b)=0$. Since

$$
\left(\begin{array}{rr}
\lambda & \lambda b \\
b & 1
\end{array}\right)\left(\begin{array}{l}
Z_{T^{\prime},+u}(\lambda, b) \\
Z_{T^{\prime},-u}(\lambda, b)
\end{array}\right)=\left(\begin{array}{l}
Z_{T,+v}(\lambda, b) \\
Z_{T,-v}(\lambda, b)
\end{array}\right)
$$

and since the matrix is invertible (as $|b| \neq 1$ ), this would imply $Z_{T^{\prime},+u}(\lambda, b)=Z_{T^{\prime},-u}(\lambda, b)=0$ and hence $Z_{T^{\prime}}(\lambda, b)=0$, contradicting the minimality of $T$. Therefore, $R_{T, v}=-1$.

Since the map $z \mapsto \xi(z):=\frac{Z_{T,+v}(z, b)}{Z_{T,-v}(z, b)}$ is holomorphic near $z=\lambda$, it follows that there exists $\lambda^{\prime \prime} \in \mathbb{S}_{\mathbb{Q}}$ arbitrarily close to $\lambda^{\prime}$ such that $\xi=\xi\left(\lambda^{\prime \prime}\right) \in \operatorname{Arc}\left(\lambda_{1}(b), \overline{\lambda_{1}(b)}\right) \backslash\{-1\}$. Therefore, by Lemma 14 and Theorem 12, the orbit $\left\{f_{\xi, 1}^{n}(1)\right\}$ is dense in $\mathbb{S}$. So from Lemma 4, by using paths with the tree $T$ attached to all but one of its vertices at the root $v$ of $T$, we obtain a collection of trees contained in $\mathcal{T}_{d+1}$ whose fields are dense in $\mathbb{S}$.

We next prove part (a) for all $d \geq 2$ and $b \in\left(\frac{d-2}{d}, \frac{d-1}{d+1}\right]$. The case $b \in\left(0, \frac{d-2}{d}\right]$ follows from this by invoking smaller values for $d$. Let $\lambda \in \mathbb{S}_{\mathbb{Q}} \backslash\{ \pm 1\}$. 
Let $k_{0}=d$ and $m_{0}=0$, and define the sequences $k_{n}$ and $m_{n}$ by $k_{n+1}=\left\lfloor\frac{k_{n}}{2}\right\rfloor$ and $m_{n+1}=m_{n}+k_{n+1}$. Inductively we show that $m_{n} \leq d-k_{n}$ : we have $m_{0}=d-k_{0}$ and then

$$
m_{n+1}=m_{n}+k_{n+1} \leq d-k_{n}+k_{n+1}=d-\left(k_{n}-\left\lfloor\frac{k_{n}}{2}\right\rfloor\right) \leq d-\left\lfloor\frac{k_{n}}{2}\right\rfloor=d-k_{n+1} .
$$

Clearly, there is an integer $N$ such that $k_{N+1}=1$. We claim that for every $n \in\{0, \ldots, N\}$ there is a rooted tree in $\mathcal{T}_{d+1}$ with root degree $m_{n}$ that implements a field $\xi_{n}$ so that $\left|f^{\prime}\left(\xi_{n}\right)\right|>1$ and at least one of the following holds:

1. There is a tree in $\mathcal{T}_{d+1}$ with root degree at most $m_{n}$ that implements a field inside $\operatorname{Arc}\left(\lambda_{k_{n+1}}, \overline{\lambda_{k_{n+1}}}\right) \backslash$ $\{-1\}$, or else

2. the set of fields implemented by trees in $\mathcal{T}_{d+1}$ is dense in $\mathbb{S}$.

To show this for $n=0$, we consider the tree consisting of a single vertex. This tree implements the field $\lambda$ and its root degree is 0 . By equation (2) of Lemma 10 and since $b \leq \frac{d-1}{d+1}$, we have that $\left|f_{d}^{\prime}(z)\right|>1$ for all $z \in \mathbb{S} \backslash\{1\}$ and, in particular, we have $\left|f_{d}^{\prime}(\lambda)\right|>1$. If $\lambda \in \operatorname{Arc}\left[\overline{\lambda_{k_{1}}}, \lambda_{k_{1}}\right]$, then we apply Lemma 17 to obtain Item (2). If $\lambda \in \operatorname{Arc}\left(\lambda_{k_{1}}, \overline{\lambda_{k_{1}}}\right)$, then the tree consisting of a single vertex satisfies the conditions of Item (1).

Now suppose that we have shown the claim for $n-1$ for some $n \geq 1$ and assume that we are in the case of Item (1) (otherwise, we are done); that is, there is a tree in $\mathcal{T}_{d+1}$ with root degree $m_{n-1}$ that implements a field $\xi$ inside $\operatorname{Arc}\left(\lambda_{k_{n}}, \overline{\lambda_{k_{n}}}\right) \backslash\{-1\}$. We can apply Lemma 16 to obtain a tree $T$ in $\mathcal{T}_{d+1}$ with root degree at most $k_{n}+m_{n-1}=m_{n}$ that implements a field $\zeta \neq-1$ such that $\left|f_{k_{n}}^{\prime}(\zeta)\right|>1$. If $\zeta \in \operatorname{Arc}\left[\overline{\lambda_{k_{n+1}}}, \lambda_{k_{n+1}}\right]$, we can apply Lemma 17 to obtain Item (2). We can apply this lemma because $m_{n} \leq d-k_{n}$. If $\zeta \in \operatorname{Arc}\left(\lambda_{k_{n+1}}, \overline{\lambda_{k_{n+1}}}\right)$, then $T$ itself satisfies the conditions of Item (1), which proves the claim.

To finish the proof, it remains to consider the case of Item (1), where we can find a tree in $\mathcal{T}_{d+1}$ with root degree at most $m_{N}<d-1$ which implements a field $\xi$ inside $\operatorname{Arc}\left(\lambda_{1}, \overline{\lambda_{1}}\right) \backslash\{-1\}$. We have shown in Lemma 14 that $f_{\xi, 1}$ is conjugate to an irrational rotation and thus the orbit $\left\{f_{\xi, 1}^{n}(1)\right\}_{n \geq 1}$ is dense in $\mathbb{S}$. The elements of this orbit correspond to rooted trees in $\mathcal{T}_{d+1}$ and, hence, we can conclude Item (2) in this case as well.

Let $\mathcal{R}$ denote the set of all fields implemented by rooted trees in $\mathcal{T}_{d+1}$. Let $\zeta \in \mathcal{R}$ and $T$ be a tree in $\mathcal{T}_{d+1}$ that implements $\zeta$. We construct the tree $\tilde{T}$ with root $r$ obtained by attaching $r$ to the root of $T$ with an edge. Then, the root of $\tilde{T}$ has degree 1 and the field implemented by $\tilde{T}$ is $f_{\lambda, 1}(\zeta)$. So the set of fields implemented by rooted trees in $\mathcal{T}_{d+1}$ whose root degrees are 1 contains $f_{\lambda, 1}(\mathcal{R})$. Since $f_{\lambda, 1}(\mathbb{S})=\mathbb{S}$ and $\mathcal{R}$ is dense in $\mathbb{S}$, we conclude that $f_{\lambda, 1}(\mathcal{R})$ is dense in $\mathbb{S}$ as well.

Remark 18. We note that our proof of part (b) rests on the existence of zeros for trees proved in [42], which in turn depends on the chaotic behaviour of the map $f_{d, \lambda}$. Alternatively, one could also prove part (b) directly from Lemma 15 . The same proof also yields a dense set of $\lambda \in \mathbb{S}_{\mathbb{Q}}$ for which the collection of fields of trees in $\mathcal{T}_{d+1}$ with root degree 1 is dense in $\mathbb{S}$ when $b \in\left(0, \frac{d-1}{d+1}\right]$.

\section{Contracting maps that cover via degree-derivative interplay}

In this section, we adapt the contracting/covering maps framework of [6] in our setting and show how to apply it using the degree-derivative inteplay alluded to in Subsection 2.3. Subsection 4.1 gives the details of the framework, and Subsection 4.2 gives the main lemmas that exploit this interplay.

\subsection{Density on circular arcs via contracting maps that cover}

The contracting maps that cover framework is captured by the following lemma on the interval $[0,1]$, which yields Corollary 20 on circular arcs of the unit circle $\mathbb{S}$. 
Lemma 19. Let $f_{1}, \ldots, f_{k}$ be continuously differentiable maps from the interval $[0,1]$ to itself such that $0<f_{m}^{\prime}(x)<1$ for each index $m$ and $x \in(0,1)$ and such that $\bigcup_{m=1}^{k} f_{m}([0,1])=[0,1]$.

Then for any open interval $J \subseteq[0,1]$, there is a sequence of indices $m_{1}, \ldots, m_{N}$ such that

$$
\left(f_{m_{1}} \circ \cdots \circ f_{m_{N}}\right)([0,1]) \subset J .
$$

Proof. For $m \in\{1, \ldots, k\}$, define the closed interval $I_{m}=f_{m}([0,1])=\left[f_{m}(0), f_{m}(1)\right]$ and note that $f_{m}:[0,1] \rightarrow I_{m}$ is bijective with a differentiable inverse. We define a sequence of intervals in the following way. Let $J_{0}=J$, and as long as there exists an index $m$ such that $J_{n} \subseteq I_{m}$, we define $J_{n+1}=f_{m}^{-1}(J)$. We will show that this cannot be done indefinitely; that is, there will be some interval $J_{n}$ such that $J_{n} \nsubseteq I_{m}$ for all $m$.

For an interval $I \subseteq[0,1]$, let $\ell(I)$ denote the length of the interval and denote $\ell(J)$ by $\epsilon$. For each index $m$ choose a partition $I_{m}=I_{m, L} \cup I_{m, M} \cup I_{m, R}$, where $I_{m, L}, I_{m, M}, I_{m, R}$ are of the form $\left[f_{m}(0), a\right),[a, b],\left(b, f_{m}(1)\right]$, respectively, for a choice of $a, b \in \operatorname{int}\left(I_{m}\right)$ such that $a<b$ and both $\ell\left(I_{m, L}\right)$ and $\ell\left(I_{m, R}\right)$ are less than $\epsilon / 4$. We can choose $C>1$ such that $f_{m}^{-1^{\prime}}(x)>C$ for all indices $m$ and $x \in I_{m, M}$. We will show inductively that for all $n \geq 0$ for which $J_{n}$ is defined it is the case that $\ell\left(J_{n}\right) \geq \epsilon \cdot\left(1+C^{n}\right) / 2$. For $n=0$ the statement is true. Suppose that the statement is true for $n \geq 0$ for which $J_{n+1}$ is defined. By definition, there is an index $m$ such that $J_{n} \subseteq I_{m}$ and $J_{n+1}=f_{m}^{-1}\left(J_{n}\right)$. We find

$$
\begin{aligned}
\ell\left(J_{n+1}\right) & =\ell\left(f_{m}^{-1}\left(J_{n} \cap I_{m, M}\right)\right)+\ell\left(f_{m}^{-1}\left(J_{n} \cap I_{m, L}\right)\right)+\ell\left(f_{m}^{-1}\left(J_{n} \cap I_{m, R}\right)\right) \\
& \geq C \cdot \ell\left(J_{n} \cap I_{m, M}\right)+\ell\left(J_{n} \cap I_{m, L}\right)+\ell\left(J_{n} \cap I_{m, R}\right) \\
& =C\left(\ell\left(J_{n}\right)-\ell\left(J_{n} \cap I_{m, L}\right)-\ell\left(J_{n} \cap I_{m, R}\right)\right)+\ell\left(J_{n} \cap I_{m, L}\right)+\ell\left(J_{n} \cap I_{m, R}\right),
\end{aligned}
$$

where we have used that $f_{m}^{-1^{\prime}}(x) \geq 1$ for $x \in I_{m}$. Because $\ell\left(J_{n} \cap I_{m, L}\right)+\ell\left(J_{n} \cap I_{m, R}\right) \leq \epsilon / 2$, this is again at least equal to

$$
C\left(\ell\left(J_{n}\right)-\epsilon / 2\right)+\epsilon / 2 \geq C\left(\epsilon \cdot\left(1+C^{n}\right) / 2-\epsilon / 2\right)+\epsilon / 2=\epsilon \cdot\left(1+C^{n+1}\right) / 2 .
$$

It follows that there is an index $n$ such that $J_{n}$ is not totally contained inside $I_{m}$ for any index $m$. This means that there is an $m$ such that $J_{n}$ contains at least one of the endpoints of $I_{m}$. Without loss of generality, we can assume that $J_{n}$ contains the left endpoint of $I_{m}$. It follows that there is an $a>0$ such that $f_{m}([0, a]) \subset J_{n}$ and thus there is a sequence $m_{1}, \ldots, m_{n}$ such that $\left(f_{m_{1}} \circ \cdots \circ f_{m_{n}} \circ f_{m}\right)([0, a]) \subset J$. We complete the proof by showing that for at least one of the maps $f_{i}$ there is an index $N_{a}$ for any $a>0$ such that $f_{i}^{N_{a}}([0,1]) \subset[0, a]$.

Observe that there must be at least one map $f_{i}$ such that $f_{i}(0)=0$. We obtain an inclusion of intervals $[0,1] \supset f_{i}([0,1]) \supset f_{i}^{2}([0,1]) \supset \cdots$, where $f_{i}^{N}([0,1])=\left[0, f_{i}^{N}(1)\right]$. This shows that the sequence $\left\{f_{i}^{N}(1)\right\}_{N \geq 0}$ is decreasing and thus has a limit $L$. If $L \neq 0$, we would have $f_{i}([0, L])=[0, L]$, which contradicts the fact that $f_{i}^{\prime}(x)<1$ for all $x \in(0, L)$, so $L=0$. This concludes the proof.

Corollary 20. Let $A \subset \mathbb{S}$ be a closed circular arc and let $f_{1}, \ldots, f_{k}$ be orientation-preserving continuously differentiable maps from $A$, such that $\bigcup_{m=1}^{k} f_{m}(A)=A$ and $0<\left|f_{m}^{\prime}(x)\right|<1$ for each index $m$ and $x \in A$ not equal to either of the endpoints of $A$.

Then for any open circular arc $J \subseteq A$ there is a sequence of indices $m_{1}, \ldots, m_{N}$ such that

$$
\left(f_{m_{1}} \circ \cdots \circ f_{m_{N}}\right)(A) \subset J .
$$

\subsection{Exploiting the dependence of derivatives on the degrees}

In this section, we show a few key lemmas that demonstrate how we employ the contracting maps that cover idea, by exploiting the dependence of derivatives on the degrees.

Lemma 21. Let $k \in \mathbb{Z}_{\geq 1}$ and $b \in\left[\frac{k}{k+2}, 1\right)$. Let $\xi \in \operatorname{Arc}\left[\overline{\lambda_{k+1}}, \lambda_{k+1}\right]$ with $\xi \neq 1$ be such that $\left|f_{2 k+1}^{\prime}\left(R_{k}(\xi)\right)\right| \geq 1$. Then there is an arc A of $\mathbb{S}$ such that the orbit of 1 under the action of the semigroup generated by $f_{\xi, k+1}$ and $f_{\xi, k}$ is dense in $A$. 
Proof. We can assume that $\xi$ lies in the upper half-plane. Since all maps in this argument use the parameter $\xi$, we will write $f_{m}$ instead of $f_{\xi, m}$ for all $m$. Define the $\operatorname{arc} A=\operatorname{Arc}\left[R_{k}(\xi), R_{k+1}(\xi)\right]$. Using equation (2), we find that for every $m$,

$$
\left|f_{m}^{\prime}\left(R_{k}(\xi)\right)\right|=\frac{m}{2 k+1} \cdot\left|f_{2 k+1}^{\prime}\left(R_{k}(\xi)\right)\right| \geq \frac{m}{2 k+1} .
$$

By using the fact that $R_{k+1}(\xi)$ is either a parabolic or an attracting fixed point of $f_{k+1}$, we deduce that for all $z \in A$,

$$
\left|f_{k}^{\prime}(z)\right|<\left|f_{k+1}^{\prime}(z)\right| \leq\left|f_{k+1}^{\prime}\left(R_{k+1}(\xi)\right)\right| \leq 1,
$$

where the second inequality is strict when $z \neq R_{k+1}(\xi)$. It follows that for all $z \in A$ not equal to $R_{k+1}(\xi)$ we have $k /(2 k+1) \leq\left|f_{k}^{\prime}(z)\right|<1$ and $(k+1) /(2 k+1) \leq\left|f_{k+1}^{\prime}(z)\right|<1$. Therefore,

$$
\ell(A)>\ell\left(f_{k}(A)\right)>\frac{k}{2 k+1} \ell(A) \quad \text { and } \quad \ell(A)>\ell\left(f_{k+1}(A)\right)>\frac{k+1}{2 k+1} \ell(A) .
$$

From this we deduce that $\ell\left(f_{k}(A)\right)+\ell\left(f_{k+1}(A)\right)>\ell(A)$. Thus, because $f_{k}(A)$ is of the form $\operatorname{Arc}\left[R_{k}(\xi), a\right]$ and $f_{k+1}(A)$ is of the form $\operatorname{Arc}\left[b, R_{k+1}(\xi)\right]$ for some $a, b \in A$, we conclude that $f_{k}(A) \cup f_{k+1}(A)=A$.

It follows from item (iv) of Lemma 10 that there is some $M$ such that $f_{k+1}^{M}(1) \in A$. Let $J \subseteq A$ be any open arc. According to Corollary 20, there is a sequence of indices $m_{1}, \ldots, m_{N} \in\{k, k+1\}$ such that $\left(f_{m_{1}} \circ \cdots \circ f_{m_{N}} \circ f_{k+1}^{M}\right)(1) \in J$. The fact that $J$ was chosen as an arbitrary open arc in $A$ concludes the proof.

Lemma 22. Let $k \in \mathbb{Z}_{\geq 1}$ and $b \in\left[\frac{k-1}{k+1}, 1\right)$. Let $\xi_{1}, \xi_{2} \in \operatorname{Arc}\left[\overline{\lambda_{k}}, \lambda_{k}\right]$ such that $\xi_{1}, \xi_{2}$ are distinct and lie in the same half-plane; that is, both in the upper or lower half-plane and such that $\left|f_{2 k}^{\prime}\left(R_{k}\left(\xi_{i}\right)\right)\right| \geq 1$ for $i \in\{1,2\}$. Then there is an arc $A$ of $\mathbb{S}$ such that the orbit of 1 under the action of the semigroup generated by $f_{\xi_{1}, k}$ and $f_{\xi_{2}, k}$ is dense in $A$.

Proof. We can assume that $\xi_{1}$ and $\xi_{2}$ lie in the upper half-plane and that $\operatorname{Arg}\left(\xi_{1}\right)<\operatorname{Arg}\left(\xi_{2}\right)$. Let $A=\operatorname{Arc}\left[R_{k}\left(\xi_{1}\right), R_{k}\left(\xi_{2}\right)\right]$; then for all $z \in A$ we have

$$
\frac{1}{2} \leq \frac{1}{2} \cdot\left|f_{2 k}^{\prime}\left(R_{k}\left(\xi_{1}\right)\right)\right|=\left|f_{k}^{\prime}\left(R_{k}\left(\xi_{1}\right)\right)\right| \leq\left|f_{k}^{\prime}(z)\right| \leq\left|f_{k}^{\prime}\left(R_{k}\left(\xi_{2}\right)\right)\right| \leq 1,
$$

where the second to last inequality is strict when $z \neq R_{k}\left(\xi_{2}\right)$. Therefore, for $i \in\{1,2\}$ we have

$$
\ell(A)>\ell\left(f_{\xi_{i}, k}(A)\right)>\frac{1}{2} \cdot \ell(A),
$$

and from this we deduce that $\ell\left(f_{\xi_{1}, k}(A)\right)+\ell\left(f_{\xi_{2}, k}(A)\right)>\ell(A)$. The rest of the proof proceeds exactly as the proof of Lemma 21.

Lemma 23. Let $k \geq 5, b \in\left(\frac{k-1}{k+1}, 1\right)$ and $\xi \in \operatorname{Arc}\left[\overline{\lambda_{k}}, \lambda_{k}\right]$ with $\xi \neq 1$ such that there is an integer $2 k \leq p \leq 3 k-5$ for which $\left|f_{p}^{\prime}(\xi)\right| \geq 1$. Then at least one of the following two statements holds:

(i) The orbit of 1 under the action of the semigroup generated by $f_{\xi, k-2}, f_{\xi, k-1}$ and $f_{\xi, k}$ is dense in an $\operatorname{arc}$ of $\mathbb{S}$.

(ii) We have $\left|f_{k}^{\prime}\left(R_{k}(\xi)\right)\right|>1-\frac{p-k+2}{p} \cdot \frac{p-2 k+1}{k}$.

Proof. W.l.o.g., we may assume that $\xi$ lies in the upper half plane. We write $f_{m}=f_{\xi, m}$ for all indices $m$. Define the $\operatorname{arcs} A_{1}=\operatorname{Arc}\left[R_{k-2}(\xi), R_{k-1}(\xi)\right]$ and $A_{2}=\operatorname{Arc}\left[R_{k-1}(\xi), R_{k}(\xi)\right]$. Analogous to the 
proofs of Lemmas 21 and 22, we can use Corollary 20 to show that the orbit of 1 under the action of the semigroup generated by $f_{k-2}, f_{k-1}$ and $f_{k}$ is dense in $A_{1} \cup A_{2}$ if

$$
f_{k-2}\left(A_{1} \cup A_{2}\right) \cup f_{k-1}\left(A_{1} \cup A_{2}\right) \cup f_{k}\left(A_{1} \cup A_{2}\right)=A_{1} \cup A_{2} .
$$

We will assume that this is not the case and show that this leads to statement (ii). First, we will show that the left-hand side of equation (8) does cover $A_{1}$. For any arc $A$ in the upper half-plane such that $\operatorname{Arg}(x) \geq \operatorname{Arg}(\xi)$ for all $x \in A$ and index $m$, we have

$$
\ell\left(f_{m}(A)\right)>\left|f_{m}^{\prime}(\xi)\right| \cdot \ell(A)=\frac{m \cdot\left|f_{p}^{\prime}(\xi)\right|}{p} \cdot \ell(A) \geq \frac{m}{p} \cdot \ell(A) .
$$

We use this and the fact that $\ell\left(A_{2}\right) \geq \ell\left(A_{1}\right)$, which follows from item (v) of Lemma 10 , to conclude the following:

$$
\begin{aligned}
\ell\left(f_{k-2}\left(A_{1} \cup A_{2}\right)\right)+\ell\left(f_{k-1}\left(A_{1}\right)\right) & \geq \frac{k-2}{p} \ell\left(A_{1} \cup A_{2}\right)+\frac{k-1}{p} \ell\left(A_{1}\right) \\
& \geq \frac{2(k-2)}{p} \ell\left(A_{1}\right)+\frac{k-1}{p} \ell\left(A_{1}\right) \\
& =\frac{3 k-5}{p} \ell\left(A_{1}\right) \geq \ell\left(A_{1}\right) .
\end{aligned}
$$

Because $f_{k-2}\left(A_{1} \cup A_{2}\right)$ is of the form $\operatorname{Arc}\left[R_{k-2}(\xi), a\right]$ and $f_{k-1}\left(A_{1}\right)$ is of the form $\operatorname{Arc}\left[b, R_{k-1}(\xi)\right]$, we have that $A_{1}$ is covered by $f_{k-2}\left(A_{1} \cup A_{2}\right) \cup f_{k-1}\left(A_{1}\right)$. Our assumption can be formulated as

$$
\ell\left(A_{2}\right) \geq \ell\left(f_{k-1}\left(A_{2}\right)\right)+\ell\left(f_{k}\left(A_{1} \cup A_{2}\right)\right) .
$$

Note that

$$
\ell\left(f_{k-1}\left(A_{2}\right)\right)+\ell\left(f_{k}\left(A_{1} \cup A_{2}\right)\right) \geq \frac{k-1}{p} \ell\left(A_{2}\right)+\frac{k}{p}\left(\ell\left(A_{1}\right)+\ell\left(A_{2}\right)\right) .
$$

Combining the previous two inequalities, we get

$$
\ell\left(A_{1}\right) \leq \frac{p-2 k+1}{k} \cdot \ell\left(A_{2}\right) .
$$

Let $A_{0}=\operatorname{Arc}\left[1, R_{k-2}(\xi)\right]$. By using the fact that $R_{m}(\xi)$ is a fixed point of $f_{m}$ and $f_{m}(1)=\xi$ for every $m$, we see that $f_{k-2}\left(A_{0}\right)=\operatorname{Arc}\left[\xi, R_{k-2}(\xi)\right], f_{k-1}\left(A_{0} \cup A_{1}\right)=\operatorname{Arc}\left[\xi, R_{k-1}(\xi)\right]$ and $f_{k}\left(A_{0} \cup A_{1} \cup A_{2}\right)=$ $\operatorname{Arc}\left[\xi, R_{k}(\xi)\right]$. It follows from the relation between the derivative of different maps given in item (i) of Lemma 10 that for any arc $A$ on which $f_{m_{1}}$ and $f_{m_{2}}$ are injective we have

$$
\ell\left(f_{m_{1}}(A)\right)=m_{1} \cdot \ell\left(f_{1}(A)\right)=\frac{m_{1}}{m_{2}} \cdot \ell\left(f_{m_{2}}(A)\right) .
$$

These observations can be used to write $\ell\left(A_{1}\right)$ and $\ell\left(A_{2}\right)$ as follows:

$$
\begin{aligned}
\ell\left(A_{1}\right) & =\ell\left(f_{k-1}\left(A_{0} \cup A_{1}\right)\right)-\ell\left(f_{k-2}\left(A_{0}\right)\right) \\
& =\frac{k-2}{k-1} \ell\left(f_{k-1}\left(A_{0} \cup A_{1}\right)\right)+\frac{1}{k-1} \ell\left(f_{k-1}\left(A_{0} \cup A_{1}\right)\right)-\frac{k-2}{k-1} \ell\left(f_{k-1}\left(A_{0}\right)\right) \\
& =\frac{k-2}{k-1} \ell\left(f_{k-1}\left(A_{1}\right)\right)+\frac{1}{k-1} \ell\left(f_{k-1}\left(A_{0} \cup A_{1}\right)\right)
\end{aligned}
$$


and

$$
\ell\left(A_{2}\right)=\ell\left(f_{k}\left(A_{0} \cup A_{1} \cup A_{2}\right)\right)-\ell\left(f_{k-1}\left(A_{0} \cup A_{1}\right)\right)=\ell\left(f_{k}\left(A_{2}\right)\right)+\frac{1}{k-1} \cdot \ell\left(f_{k-1}\left(A_{0} \cup A_{1}\right)\right) .
$$

By considering our way of writing $\ell\left(A_{1}\right)$ and the inequalities given in (9) and (10), we obtain the following inequalities:

$$
\begin{aligned}
\frac{1}{k-1} \cdot \ell\left(f_{k-1}\left(A_{0} \cup A_{1}\right)\right) & =\ell\left(A_{1}\right)-\frac{k-2}{k-1} \cdot \ell\left(f_{k-1}\left(A_{1}\right)\right)<\ell\left(A_{1}\right)-\frac{k-2}{k-1} \cdot \frac{k-1}{p} \ell\left(A_{1}\right) \\
& =\frac{p-k+2}{p} \cdot \ell\left(A_{1}\right)<\frac{p-k+2}{p} \cdot \frac{p-2 k+1}{k} \cdot \ell\left(A_{2}\right) .
\end{aligned}
$$

It follows from the fact that $\operatorname{Arg}\left(R_{k}(\xi)\right) \geq \operatorname{Arg}(x)$ for all $x \in A_{2}$ that $\ell\left(f_{k}\left(A_{2}\right)\right)<\left|f_{k}^{\prime}\left(R_{k}(\xi)\right)\right| \cdot \ell\left(A_{2}\right)$. By using this inequality and the previous inequality, we obtain

$$
\begin{aligned}
\ell\left(A_{2}\right) & =\ell\left(f_{k}\left(A_{2}\right)\right)+\frac{1}{k-1} \cdot \ell\left(f_{k-1}\left(A_{0} \cup A_{1}\right)\right) \\
& <\left|f_{k}^{\prime}\left(R_{k}(\xi)\right)\right| \cdot \ell\left(A_{2}\right)+\frac{p-k+2}{p} \cdot \frac{p-2 k+1}{k} \cdot \ell\left(A_{2}\right) .
\end{aligned}
$$

We can cancel $\ell\left(A_{2}\right)$ and rewrite to obtain

$$
\left|f_{k}^{\prime}\left(R_{k}(\xi)\right)\right|>1-\frac{p-k+2}{p} \cdot \frac{p-2 k+1}{k},
$$

which is what we set out to prove.

Corollary 24. Let $m$ be a positive integer, $b \in\left(\frac{m-1}{m+1}, 1\right)$ and $\xi \in \operatorname{Arc}\left[\overline{\lambda_{m}}, \lambda_{m}\right]$ with $\xi \neq 1$ such that either of the following holds:

(a) $m \geq 8$ and $\left|f_{2 m}^{\prime}(\xi)\right| \geq 1$;

(b) $m \geq 9$ and $\left|f_{2 m+1}^{\prime}(\xi)\right| \geq 1$.

Then the orbit of 1 under the action of the semigroup generated by $f_{\xi, m-3}, f_{\xi, m-2}, f_{\xi, m-1}$ and $f_{\xi, m}$ is dense in an arc of $\mathbb{S}$.

Proof. We will again assume that $\xi$ lies in the upper half-plane. We apply Lemma 23 with $k=m-1$ and $p=2 m$ for item (a) and $p=2 m+1$ for item (b). If the first statement of that lemma holds, we see that orbit of 1 under the action of $f_{m-3}, f_{m-2}$ and $f_{m-1}$ generates an arc, in which case we are done. If we assume that the second statement holds, we obtain

$$
f_{m-1}^{\prime}\left(R_{m-1}(\xi)\right)>1-\frac{2 m-(m-1)+2}{2 m} \cdot \frac{2 m-2(m-1)+1}{m-1}>\frac{1}{2}
$$

in the case where $p=2 m$ and

$$
f_{m-1}^{\prime}\left(R_{m-1}(\xi)\right)>1-\frac{2 m+1-(m-1)+2}{2 m+1} \cdot \frac{2 m+1-2(m-1)+1}{m-1}>\frac{1}{2}
$$

in the case where $p=2 m+1$. It follows that for $x \in \operatorname{Arc}\left[R_{k-1}(\xi), R_{k}(\xi)\right]$, we obtain $1>\left|f_{m}^{\prime}(x)\right|>$ $\left|f_{m-1}^{\prime}(x)\right|>1 / 2$. Therefore, with $A=\operatorname{Arc}\left[R_{k-1}(\xi), R_{k}(\xi)\right]$, we get

$$
\ell\left(f_{m}(A)\right)+\ell\left(f_{m-1}(A)\right) \geq \ell(A) .
$$

This, together with Corollary 20, implies that the orbit of 1 under the action of the semigroup generated by $f_{m-1}$ and $f_{m}$ is dense in $A$. 


\section{Proof of Lemma 17 for Some Special Cases}

The arguments of this section will be used to cover some leftover cases in the proof of Lemma 17 that are not directly covered by the results of the previous section.

\subsection{Proof of Lemma 17 for powers of 2}

The following lemma will be used in the proof of Lemma 17 for those values of $k$ for which either $k$ or $k+1$ is a power of 2; see the proof in Section 6 for details.

Lemma 25. Let $d \geq 2, k \geq 0$ be integers, $b \in\left(0, \frac{d-1}{d+1}\right] \cap \mathbb{Q}, \lambda \in \mathbb{S}_{\mathbb{Q}} \backslash\{ \pm 1\}$ and $\xi \in \Lambda_{2^{k}}(b) \cap \mathbb{S}_{\mathbb{Q}}$ with $\xi \neq \pm 1$. Suppose there is a tree in $\mathcal{T}_{d+1}$ with root degree at most $d-\left(2^{k+1}-1\right)$ that implements the field $\xi$. Then the set of fields implemented by rooted trees in $\mathcal{T}_{d+1}$ is dense in $\mathbb{S}$.

Proof. We will prove this by induction on $k$. For $k=0$, the field $\xi$ has to lie in $\operatorname{Arc}\left(\lambda_{1}, \overline{\lambda_{1}}\right) \backslash\{-1\}$ and the root degree of the tree in $\mathcal{T}_{d+1}$ implementing $\xi$ is at most $d-1$. From Corollary 13 and Lemma 14, we have that $f_{\xi, 1}$ is conjugate to an irrational rotation and thus the orbit of any initial point $z_{0} \in \mathbb{S}$ is dense in $\mathbb{S}$. By Lemma 4, every element of the set $\left\{f_{\xi, 1}^{n}(\lambda)\right\}_{n \geq 1}$ is the field implemented by a tree in $\mathcal{T}_{d+1}$ and hence we obtain the theorem for $k=0$.

Now suppose that $k \geq 1$ and that we have proved the statement for $k-1$. If $b<\left(2^{k-1}-1\right) /\left(2^{k-1}+1\right)$, then we must have $k>1$, and by Lemma 8 we can immediately apply the induction hypothesis with $\xi=\lambda$ and tree consisting of a single vertex. So, assume that $b \geq\left(2^{k-1}-1\right) /\left(2^{k-1}+1\right)$ and observe that the parameter $\lambda_{2^{k-1}} \in \mathbb{S}$ from Lemma 10 exists. It follows from Lemma 16 that there is $\sigma \in \mathbb{S}$ with $\left|f_{2^{k}}^{\prime}(\sigma)\right|>1$ and a set $\mathcal{R}=\left\{\zeta_{n}\right\}_{n \geq 1}$ accumulating on $\sigma$ such that each $\zeta_{n}$ is implemented by a tree in $\mathcal{T}_{d+1}$ whose root degree is at most $d-\left(2^{k+1}-1\right)+2^{k}=d-\left(2^{k}-1\right)$. If $\mathcal{R}$ has a nonempty intersection with $\operatorname{Arc}\left(\lambda_{2^{k-1}}, \overline{\lambda_{2^{k-1}}}\right) \backslash\{-1\}$, we can apply the induction hypothesis to the tree corresponding to the field in this intersection. Therefore, we assume that the elements of $\mathcal{R}$ accumulate on $\sigma$ from inside $\operatorname{Arc}\left[\overline{\lambda_{2^{k-1}}}, \lambda_{2^{k-1}}\right]$. It follows that we can find two distinct elements $r_{1}, r_{2} \in \mathcal{R}$ such that they both lie in either $\operatorname{Arc}\left(\overline{\lambda_{2^{k-1}}}, 1\right)$ or $\operatorname{Arc}\left(1, \lambda_{2^{k-1}}\right)$ and such that $\left|f_{2^{k}}^{\prime}\left(r_{i}\right)\right|>1$ for $i=1,2$. By Remark 11, we have $\left|f_{2^{k}}^{\prime}\left(R_{2^{k-1}}\left(r_{i}\right)\right)\right|>\left|f_{2^{k}}^{\prime}\left(r_{i}\right)\right|>1$ and thus we can apply Lemma 22 to conclude that the following set is dense in an $\operatorname{arc} A$ of the circle:

$$
\mathcal{A}=\left\{\left(f_{r_{i_{1}}, 2^{k-1}} \circ \cdots \circ f_{r_{i_{n}}, 2^{k-1}}\right)(1): n \in \mathbb{Z}_{\geq 1} \text { and } i_{1}, \ldots, i_{n} \in\{1,2\}\right\} .
$$

Since $r_{1}, r_{2}$ are implemented by trees in $\mathcal{T}_{d+1}$ whose root degrees are at most $d-\left(2^{k+1}-1\right)+2^{k}=$ $d-\left(2^{k}-1\right)$, by Lemma 4 , every element of $\mathcal{A}$ is implemented by a tree in $\mathcal{T}_{d+1}$ whose root degree is bounded by $d-\left(2^{k}-1\right)+2^{k-1}=d-\left(2^{k-1}-1\right) \leq d$. We have seen that (2) implies that for $b \leq(d-1) /(d+1)$ it holds that $\left|f_{d}^{\prime}(z)\right|>1$ for all $z \in \mathbb{S} \backslash\{1\}$. This implies that there is some $N \in \mathbb{Z}_{\geq 1}$ such that $f_{d}^{N}(A)=\mathbb{S}$. It follows that the set $\left\{f_{\lambda, d}^{N}(a): a \in \mathcal{A}\right\}$ is dense in $\mathbb{S}$, finishing the proof, since every element of this set corresponds to the field of a tree in $\mathcal{T}_{d+1}$ (again using Lemma 4 ).

\subsection{Proof of Lemma 17 for small cases}

In this section, we give the main lemma needed to cover certain small cases of Lemma 17. Interestingly, the proof uses a Cantor-style construction, explained in detail in the next subsection.

\subsubsection{Near-arithmetic progressions}

Let $\alpha \in(0,1)$ and define the maps from the unit interval to itself given by $\phi_{0}(x)=\alpha x$ and $\phi_{1}(x)=$ $\alpha x+(1-\alpha)$. Let $\Omega=\cup_{n=0}^{\infty}\{0,1\}^{n}$ be the set of finite binary sequences. For $\omega \in \Omega$ we let $|\omega|$ denote the length of $\omega$ and for $\omega_{1}, \omega_{2} \in \Omega$ we let $\omega_{1} \oplus \omega_{2} \in \Omega$ denote the concatenation of the two sequences. For $\omega \in \Omega$ of the form $\left(\omega^{1}, \ldots, \omega^{n}\right)$ and two maps $f_{0}, f_{1}$, we let $f_{\omega}=f_{\omega^{1}} \circ \cdots \circ f_{\omega^{n}}$ and if $|\omega|=0$ 
we let $f_{\omega}$ denote the identity map. The properties of the semigroup generated by $\phi_{0}$ and $\phi_{1}$ for certain parameters $\alpha$ have been studied extensively. For $\alpha \in\left(0, \frac{1}{2}\right)$, the set

$$
\mathcal{C}_{\alpha}=\bigcap_{n=0}^{\infty} \bigcup_{\substack{\omega \in \Omega \\|\omega|=n}} \phi_{\omega}([0,1])
$$

is a Cantor set, with $\mathcal{C}_{1 / 3}$ being the Cantor ternary set. We will not use the properties of Cantor sets, so we do not define them. First we state some easy-to-prove properties of this semigroup to describe a construction that will help us to prove Lemma 17 for small cases of $k$.

Lemma 26. Let $\omega \in \Omega$ and $\alpha \in(0,1)$. Then $\phi_{\omega}([0,1])$ is an interval of length $\alpha^{|\omega|}$ and, furthermore, the intervals $\phi_{\omega \oplus(0)}([0,1])$ and $\phi_{\omega \oplus(1)}([0,1])$ are subintervals of $\phi_{\omega}([0,1])$ sharing the left and right boundaries, respectively.

Proof. Because the derivative of $\phi_{i}$ is constantly equal to $\alpha$ for $i=1,2$, it follows that the length of $\phi_{\omega}([0,1])$ is $\alpha^{|\omega|}$. The maps $\phi_{i}$ are increasing and thus we can write $\phi_{\omega}([0,1])=\left[\phi_{\omega}(0), \phi_{\omega}(1)\right]$ and also $\phi_{\omega \oplus(0)}([0,1])=\left[\phi_{\omega \oplus(0)}(0), \phi_{\omega \oplus(0)}(1)\right]=\left[\phi_{\omega}(0), \phi_{\omega \oplus(0)}(1)\right]$. Therefore, the left boundaries of $\phi_{\omega}([0,1])$ and $\phi_{\omega \oplus(0)}([0,1])$ are equal. The length of the latter interval is $\alpha^{|\omega|+1}$, which is less than the length of $\phi_{\omega}([0,1])$, and thus $\phi_{\omega \oplus(0)}([0,1])$ is indeed contained in $\phi_{\omega}([0,1])$. The stated property of $\phi_{\omega \oplus(1)}([0,1])$ follows completely analogously.

For two sets $A, B \subseteq \mathbb{R}$, we will let $A+B=\{a+b: a \in A, b \in B\}$. A famous property of the Cantor ternary set is that $\mathcal{C}_{1 / 3}+\mathcal{C}_{1 / 3}=[0,2]$. More generally, one can show that $\mathcal{C}_{\alpha}+\mathcal{C}_{\alpha}=[0,2]$ for all $\alpha \in\left[\frac{1}{3}, 1\right)$. In [37], an overview is given of the possible structures of $\mathcal{C}_{\alpha_{1}}+\mathcal{C}_{\alpha_{2}}$ for pairs of $\alpha_{1}, \alpha_{2} \in(0,1)$. Similar methods to those used in [37] can be used to show the following.

Lemma 27. Let $\alpha \in\left[\frac{1}{3}, 1\right)$ and $\epsilon>0$. Then there are sequences $\omega_{1}, \omega_{2}, \omega_{3} \in \Omega$ such that for all triples $p_{1}, p_{2}, p_{3}$ with $p_{i} \in \phi_{\omega_{i}}([0,1])$,

$$
\left|\frac{p_{2}-p_{1}}{p_{3}-p_{2}}-1\right|<\epsilon .
$$

Proof. First assume that $\alpha \in\left[\frac{1}{2}, 1\right)$. Then $\phi_{0}([0,1]) \cup \phi_{1}([0,1])=[0,1]$. It follows from Lemma 19 that for any $\delta>0$ there are elements $\omega_{1}, \omega_{2}$ and $\omega_{3}$ in $\Omega$ such that

$$
\phi_{\omega_{1}}([0,1]) \subseteq[0, \delta], \quad \phi_{\omega_{2}}([0,1]) \subseteq[1 / 2-\delta, 1 / 2+\delta] \quad \text { and } \quad \phi_{\omega_{3}}([0,1]) \subseteq[1-\delta, 1] .
$$

By choosing $\delta$ small enough, we can guarantee the inequality in (11).

Assume now that $\alpha \in\left[\frac{1}{3}, \frac{1}{2}\right)$. We will first show that if there are $\omega_{i} \in \Omega$ with $\left|\omega_{i}\right|=n$ and $q_{i} \in \phi_{\omega_{i}}([0,1])$ for $i=1,2,3$ such that $q_{1}+q_{3}=2 q_{2}$, then there are choices of indices $k_{i} \in\{0,1\}$ such that there exist $\tilde{q_{i}} \in \phi_{\omega_{i} \oplus\left(k_{i}\right)}([0,1])$ for which $\tilde{q_{1}}+\tilde{q_{3}}=2 \tilde{q_{2}}$. Suppose that we are given such $\omega_{i}$ and $q_{i}$. Let $I_{i}=\phi_{\omega_{i}}([0,1])$ and $I_{i}^{k}=\phi_{\omega_{i} \oplus(k)}([0,1])$ for $i=1,2,3$ and $k=0,1$. We will show that

$$
I_{1}+I_{3}=\left(I_{1}^{0}+I_{3}^{0}\right) \cup\left(I_{1}^{1}+I_{3}^{0}\right) \cup\left(I_{1}^{0}+I_{3}^{1}\right)
$$

Let $a_{1}$ and $a_{3}$ be the left boundaries of $I_{1}$ and $I_{3}$, respectively. Because $\left|\omega_{1}\right|=\left|\omega_{3}\right|=n$, it follows that $I_{1}=\left[a_{1}, a_{1}+\alpha^{n}\right]$ and $I_{3}=\left[a_{3}, a_{3}+\alpha^{n}\right]$ and thus $I_{1}+I_{3}=\left[a_{1}+a_{3}, a_{1}+a_{3}+2 \alpha^{n}\right]$, which we can denote as $a_{1}+a_{3}+\alpha^{n} \cdot[0,2]$. Now

$$
\begin{aligned}
I_{1}^{0}+I_{3}^{0}=\left(a_{1}+\alpha^{n} \cdot[0, \alpha]\right)+\left(a_{3}+\alpha^{n} \cdot[0, \alpha]\right) & =a_{1}+a_{3}+\alpha^{n} \cdot[0,2 \alpha] \\
I_{1}^{1}+I_{3}^{0}=\left(a_{1}+\alpha^{n} \cdot[1-\alpha, 1]\right)+\left(a_{3}+\alpha^{n} \cdot[0, \alpha]\right) & =a_{1}+a_{3}+\alpha^{n} \cdot[1-\alpha, 1+\alpha] \\
I_{1}^{1}+I_{3}^{1}=\left(a_{1}+\alpha^{n} \cdot[1-\alpha, 1]\right)+\left(a_{3}+\alpha^{n} \cdot[1-\alpha, 1]\right) & =a_{1}+a_{3}+\alpha^{n} \cdot[2-2 \alpha, 2] .
\end{aligned}
$$




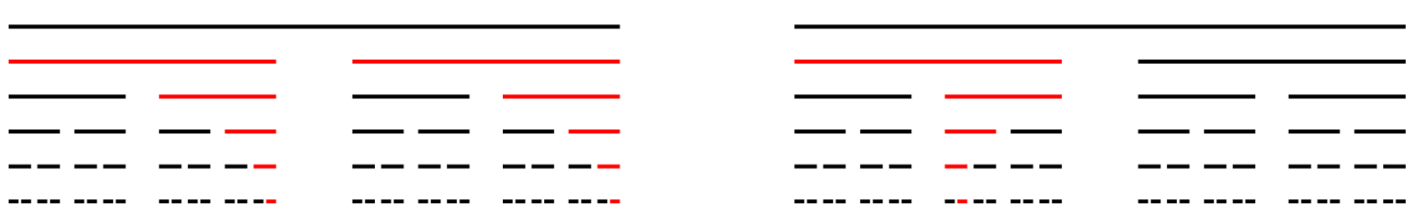

Figure 1. An illustration of the union of $\phi_{\omega}([0,1])$, where $\omega \in \Omega$ runs over all sequences of length $n$ for $n=0,1, \ldots, 6$ for $\alpha=7 / 16$. At each level, starting at level 2, three red intervals are highlighted containing elements $q_{1}, q_{2}$ and $q_{3}$, respectively, such that $q_{1}+q_{3}=2 q_{2}$.

Because $\alpha \in\left[\frac{1}{3}, 1\right)$, it follows that

$$
[0,2]=[0,2 \alpha] \cup[1-\alpha, 1+\alpha] \cup[2-2 \alpha, 2],
$$

thus showing (12). Because there are $q_{i} \in I_{i}$ such that $q_{1}+q_{3}=2 q_{2}$, we know that $I_{1}+I_{3}$ is not disjoint from $2 I_{2}$. These two intervals have the same length and thus at least one of the boundary points of $2 I_{2}$ lies in $I_{1}+I_{3}$; therefore, there is a $k_{2} \in\{0,1\}$ such that $2 I_{2}^{k_{2}}$ is not disjoint from $I_{1}+I_{3}$ because the intervals $I_{2}^{0}$ and $I_{2}^{1}$ contain the respective boundary points of $I_{2}$. This means that $2 I_{2}^{k_{2}}$ is not disjoint from $\left(I_{1}^{0}+I_{3}^{0}\right) \cup\left(I_{1}^{1}+I_{3}^{0}\right) \cup\left(I_{1}^{0}+I_{3}^{1}\right)$ and thus there are also choices of $k_{1}, k_{3} \in\{0,1\}$ such that $I_{1}^{k_{1}}+I_{3}^{k_{3}}$ is not disjoint from $2 I_{2}^{k_{2}}$. It follows that there are $\tilde{q_{i}} \in I_{i}^{k_{i}}$ such that $\tilde{q_{1}}+\tilde{q_{3}}=2 \tilde{q_{2}}$.

Let $\omega_{1}=(0,0), \omega_{2}=(0,1)$ and $\omega_{3}=(1,0)$. Note that $0 \in \phi_{\omega_{1}}([0,1])$ and $1-\alpha \in \phi_{\omega_{3}}([0,1])=$ $\left[1-\alpha, 1-\alpha+\alpha^{2}\right]$. Furthermore, it can be checked, using the fact that $\alpha \in\left[\frac{1}{3}, \frac{1}{2}\right)$, that $(1-\alpha) / 2 \in$ $\phi_{\omega_{2}}([0,1])=\left[\alpha-\alpha^{2}, \alpha\right]$, and thus there are $q_{i} \in \phi_{\omega_{i}}([0,1])$ such that $q_{1}+q_{3}-2 q_{2}=0$. From the previous considerations it follows that there are $\tilde{\omega}_{i} \in \Omega$ of arbitrary length such that there are $\tilde{q}_{i} \in \phi_{\omega_{i} \oplus \tilde{\omega}_{i}}([0,1])$ for which $\tilde{q}_{1}+\tilde{q}_{3}-2 \tilde{q}_{2}=0$. See Figure 1 for an illustration of the construction described in this proof. By taking the length of $\tilde{\omega}_{i}$ large enough, the lengths of the intervals can be made arbitrarily small, and thus we can guarantee that

$$
\left|p_{3}-p_{2}\right| \cdot\left|\frac{p_{2}-p_{1}}{p_{3}-p_{2}}-1\right|=\left|p_{1}+p_{3}-2 p_{2}\right|<\epsilon \cdot(1-2 \alpha)
$$

for all triples $p_{i} \in \phi_{\omega_{i} \oplus \tilde{\omega}_{i}}([0,1])$. Because $\phi_{\omega_{i} \oplus \tilde{\omega}_{i}}([0,1]) \subseteq \phi_{\omega_{i}}([0,1])$, we conclude that $p_{3}-p_{2}$ is at least $1-2 \alpha$. The inequality in (11) follows.

Lemma 28. Let $\alpha \in\left[\frac{1}{3}, 1\right), \epsilon>0$ and $f_{0}, f_{1}$ differentiable maps from $[0,1]$ to itself with fixed points 0 and 1 , respectively. Then there is a constant $\delta>0$ such that if $\left|f_{i}^{\prime}(x)-\alpha\right|<\delta$ for $i=0,1$ and all $x \in[0,1]$, then there are $\omega_{1}, \omega_{2}, \omega_{3} \in \Omega$ such that for all triples $p_{1}, p_{2}, p_{3}$ with $p_{i} \in f_{\omega_{i}}([0,1])$ it holds that

$$
\left|\frac{p_{2}-p_{1}}{p_{3}-p_{2}}-1\right|<\epsilon
$$

Proof. Suppose that $\left|f_{i}^{\prime}(x)-\alpha\right|<\delta$ for $i=0,1$ and all $x \in[0,1]$. For any $x \in[0,1]$, we can write

$$
f_{0}(x)=\int_{0}^{x} f_{0}^{\prime}(t) d t \quad \text { and } \quad f_{1}(x)=1-\int_{x}^{1} f_{1}^{\prime}(t) d t .
$$

We show inductively that for all $x \in[0,1]$ and $\omega \in \Omega$ we have $\left|f_{\omega}(x)-\phi_{\omega}(x)\right| \leq|\omega| \cdot \delta$. When $|\omega|=0$, the statement is clear, so we suppose that $|\omega|>0$. Assume that the first entry of $\omega$ is a 0 , so we write 
$\omega=(0) \oplus \omega^{\prime}$ for some $\omega^{\prime} \in \Omega$ with $|\omega|=\left|\omega^{\prime}\right|+1$. Let $x \in[0,1]$; we assume that we have shown that $\left|f_{\omega^{\prime}}(x)-\phi_{\omega^{\prime}}(x)\right|<\delta \cdot\left|\omega^{\prime}\right|$. We denote $f_{\omega^{\prime}}(x)$ by $y$ and $\phi_{\omega^{\prime}}(x)$ by $y+r$, where $|r| \leq \delta \cdot\left|\omega^{\prime}\right|$. Now

$$
\begin{aligned}
\left|f_{\omega}(x)-\phi_{\omega}(x)\right| & =\left|f_{0}(y)-\phi_{0}(y+r)\right|=\left|\int_{0}^{y} f_{0}^{\prime}(t) d t-\alpha \cdot(y+r)\right|=\left|\int_{0}^{y}\left(f_{0}^{\prime}(t)-\alpha\right) d t-\alpha r\right| \\
& \leq \int_{0}^{y}\left|f_{0}^{\prime}(t)-\alpha\right| d t+\alpha|r| \leq y \delta+\alpha \delta\left|\omega^{\prime}\right|<\delta\left(\left|\omega^{\prime}\right|+1\right)=\delta|\omega| .
\end{aligned}
$$

If the first entry of $\omega$ is a 1 , the calculation is analogous.

Let $\omega_{1}, \omega_{2}, \omega_{3} \in \Omega$ such that for all triples $p_{1}, p_{2}, p_{3}$ with $p_{i} \in \phi_{\omega_{i}}([0,1])$ it holds that $\left|\frac{p_{2}-p_{1}}{p_{3}-p_{2}}-1\right|<$ $\epsilon / 2$. These choices of $\omega_{i}$ exist by Lemma 27. For this inequality to hold, it must be the case that $\phi_{\omega_{2}}([0,1]) \cap \phi_{\omega_{3}}([0,1])=\emptyset$ and thus, since the map $\left(p_{1}, p_{2}, p_{3}\right) \rightarrow\left(p_{2}-p_{1}\right) /\left(p_{3}-p_{2}\right)$ is continuous in all points where $p_{2} \neq p_{3}$, we can find three open intervals $I_{1}, I_{2}, I_{3}$ with $\phi_{\omega_{i}}([0,1]) \subseteq I_{i}$ such that for all triples $q_{i} \in I_{i}$ we have

$$
\left|\frac{q_{2}-q_{1}}{q_{3}-q_{2}}-1\right|<\epsilon .
$$

We showed that by making $\delta$ small enough we obtain bounds on the difference between $f_{\omega}(x)$ and $\phi_{\omega}(x)$ uniformly over all $x \in[0,1]$ and $\omega$ of bounded length. Therefore, we can make $\delta$ sufficiently small such that $f_{\omega_{i}}([0,1]) \subset I_{i}$ for $i \in\{1,2,3\}$, which is enough to conclude the statement of the lemma.

Corollary 29. Let $\alpha \in\left[\frac{1}{3}, 1\right), \epsilon>0$. There is $\delta>0$ such that the following holds for any closed circular arc $A \subseteq \mathbb{S}^{1}$ and two maps $f_{0}, f_{1}: A \rightarrow A$ with the respective endpoints of $A$ as fixed points with the property that ||$f_{i}^{\prime}(z)|-\alpha|<\delta$ for all $z \in A$.

There exist $\omega_{1}, \omega_{2}, \omega_{3} \in \Omega$ such that for all triples $p_{i}$ with $p_{i} \in f_{\omega_{i}}(A)$ we have that $\operatorname{Arc}\left[p_{1}, p_{2}\right]$ and $\operatorname{Arc}\left[p_{2}, p_{3}\right]$ are subsets of $A$ satisfying

$$
\left|\frac{\ell\left(\operatorname{Arc}\left[p_{1}, p_{2}\right]\right)}{\ell\left(\operatorname{Arc}\left[p_{2}, p_{3}\right]\right)}-1\right|<\epsilon .
$$

We are now ready to prove the following lemma.

Lemma 30. Let $d \in \mathbb{Z}_{\geq 2}, k \in \mathbb{Z}_{\geq 1}, b \in\left[\frac{k-1}{k+1}, \frac{d-1}{d+1}\right]$ with $b \neq 0$ and $\lambda \in \mathbb{S}$. Let $\xi \in \mathbb{S} \backslash\{-1\}$ with $\left|f_{3 k}^{\prime}(\xi)\right|>1$. Let $\left\{\xi_{n}\right\}_{n \geq 1}$ be a sequence in $\mathbb{S}$ converging to $\xi$ and not equal to $\xi$ such that for all positive integers $n$ there is a rooted tree $T_{n}$ in $\mathcal{T}_{d+1}$, with root degree $m \leq d-2 k$ implementing the field $\xi_{n}$. Then at least one of the following is true:

1. The set of fields implemented by rooted trees in $\mathcal{T}_{d+1}$ is dense in $\mathbb{S}$.

2. Given $\epsilon>0$, there is a rooted tree in $\mathcal{T}_{d+1}$ with root degree at most $m+k$ that implements the field $r \in \operatorname{Arc}\left(\lambda_{k}, \overline{\lambda_{k}}\right) \backslash\{-1\}$ with $\left|f_{3 k}^{\prime}(r)\right|>\left|f_{3 k}^{\prime}(\xi)\right|-\epsilon$.

Proof. We distinguish the following three cases:

(i) $\xi \in \operatorname{Arc}\left(\lambda_{k}, \overline{\lambda_{k}}\right)$.

(ii) $\xi \in \operatorname{Arc}\left[\overline{\lambda_{k}}, \lambda_{k}\right]$ and $R_{k}(\xi) \in \operatorname{Arc}\left(\lambda_{k}, \overline{\lambda_{k}}\right)$.

(iii) $\xi \in \operatorname{Arc}\left[\overline{\lambda_{k}}, \lambda_{k}\right]$ and $R_{k}(\xi) \in \operatorname{Arc}\left[\overline{\lambda_{k}}, \lambda_{k}\right]$.

Suppose first we are in case (i). Then, since $\xi_{n} \rightarrow \xi$ and thus $f_{3 k}^{\prime}\left(\xi_{n}\right) \rightarrow f_{3 k}^{\prime}(\xi)$, given $\epsilon$, there is an integer $n$ such that $\xi_{n} \in \operatorname{Arc}\left(\lambda_{k}, \overline{\lambda_{k}}\right) \backslash\{-1\}$ and $\left|f_{3 k}^{\prime}\left(\xi_{n}\right)\right|>\left|f_{3 k}^{\prime}(\xi)\right|-\epsilon$. The rooted tree $T_{n}$ satisfies the requirements of statement (2) of the lemma.

To prove the lemma for cases (ii) and (iii), we define the following set:

$$
\mathcal{R}=\left\{f_{\xi_{n}, k}^{N}\left(\xi_{n}\right): n, N \geq 1\right\}
$$


By repeatedly applying Lemma 4, we see that every element of $\mathcal{R}$ corresponds to the field implemented by a rooted tree in $\mathcal{T}_{d+1}$ whose root degree is at most $m+k$. The following limits follow from continuity:

$$
\lim _{N \rightarrow \infty} \lim _{n \rightarrow \infty} f_{\xi_{n}, k}^{N}\left(\xi_{n}\right)=\lim _{N \rightarrow \infty} f_{\xi, k}^{N}(\xi)=R_{k}(\xi) .
$$

Therefore, $R_{k}(\xi)$ is an accumulation of $\mathcal{R}$ and, in fact, there is a sequence $\left\{\zeta_{n}\right\}_{n \geq 1}$ of elements in $\mathcal{R}$ converging to $R_{k}(\xi)$ but not equal to $R_{k}(\xi)$. If we are in case (ii), by Remark 11 we can take $\zeta_{n}$ sufficiently close to $R_{k}(\xi)$ so that $\zeta_{n} \in \operatorname{Arc}\left(\lambda_{k}, \overline{\lambda_{k}}\right) \backslash\{-1\}$. Since $\left|f_{3 k}^{\prime}\left(R_{k}(\xi)\right)\right|>\left|f_{3 k}^{\prime}(\xi)\right|$ by Remark 11 , we can further ensure that $\left|f_{3 k}^{\prime}\left(\zeta_{n}\right)\right|>\left|f_{3 k}^{\prime}(\xi)\right|$. The corresponding tree with field $\zeta_{n}$ satisfies the condition of statement (2) of the lemma.

Suppose now we are in case (iii) and suppose first that $R_{k}(\xi) \in\left\{\overline{\lambda_{k}}, \lambda_{k}\right\}$. If a subsequence $\left(\zeta_{n}\right)$ converges to $R_{k}(\xi)$ along the $\operatorname{arc} \operatorname{Arc}\left(\lambda_{k}, \overline{\lambda_{k}}\right)$, we obtain a $\zeta_{n} \in \operatorname{Arc}\left(\lambda_{k}, \overline{\lambda_{k}}\right)$ and, by the same reasoning as in the previous case, we can conclude that statement (2) of the lemma holds. So we can assume that for large enough $n$, all $\zeta_{n}$ lie in $\operatorname{Arc}\left(\overline{\lambda_{k}}, \lambda_{k}\right)$. In this case, we find that for sufficiently high $n$ the elements $\zeta_{n}$ get arbitrarily close to either $\lambda_{k}$ or $\overline{\lambda_{k}}$ and thus $\left|f_{k}^{\prime}\left(R_{k}\left(\zeta_{n}\right)\right)\right|$ gets arbitrarily close to 1 . It follows that we can find $n_{1}$ and $n_{2}$ such that $\zeta_{n_{1}}$ and $\zeta_{n_{2}}$ lie in the same half-plane and such that $\left|f_{2 k}^{\prime}\left(R_{k}\left(\zeta_{n_{i}}\right)\right)\right|>1$ for $i=1,2$. It follows then from Lemma 22 that, if we let $g_{0}=f_{\zeta_{1}, k}$ and $g_{1}=f_{\zeta_{n_{2}}, k}$, the set

$$
\mathcal{R}_{1}=\left\{g_{\omega}(1): \omega \in \Omega,|\omega| \geq 1\right\}
$$

is dense in an $\operatorname{arc} A \subseteq S$. By applying Lemma 4, we observe that every $r \in \mathcal{R}_{1}$ corresponds to the field implemented by a rooted tree in $\mathcal{T}_{d+1}$ with root degree at most $m+2 k \leq d$. Because the tree consisting of a single vertex implements the field $\lambda$, we can apply Lemma 4 to see that every element in the set

$$
\mathcal{R}_{2}=\left\{f_{\lambda, d}^{n}(r): r \in \mathcal{R}_{1}, n \geq 1\right\}
$$

corresponds to the field implemented by a rooted tree in $\mathcal{T}_{d+1}$ with root degree at most $d$. Because $b$ is chosen such that $\left|f_{d}^{\prime}(z)\right|>1$ for all $z \in \mathbb{S}-\{1\}$, we find that $f_{\lambda, d}^{N}(A)=\mathbb{S}$ for a sufficiently large $N$ and thus $\mathcal{R}_{2}$ is dense in $\mathbb{S}$, which shows that in this case statement (1) of the lemma holds.

Finally, we assume that $R_{k}(\xi) \in \operatorname{Arc}\left(\overline{\lambda_{k}}, \lambda_{k}\right)$. W.l.o.g., assume that $\xi$ lies in the upper half-plane. Let $\alpha=\left|f_{k}^{\prime}\left(R_{k}(\xi)\right)\right|$. It follows from the fact that $R_{k}(\xi) \in \operatorname{Arc}\left(\xi, \lambda_{k}\right)$ that $\alpha \in(1 / 3,1)$. Let $\epsilon_{1}, \epsilon_{2}>0$ be two reals whose value will be determined later. Let $\delta$ be the constant obtained from applying Corollary 29 to $\alpha$ and $\epsilon=\epsilon_{1}$. Now choose $n_{1}, n_{2}$ such that $\xi_{n_{1}}, \xi_{n_{2}}$ have the following properties:

(a) $\xi_{n_{1}}$ and $\xi_{n_{2}}$ lie in the upper half-plane, $\operatorname{Arg}\left(\xi_{n_{1}}\right)<\operatorname{Arg}\left(\xi_{n_{2}}\right), \operatorname{Arc}\left[R_{k}\left(\xi_{n_{1}}\right), R_{k}\left(\xi_{n_{2}}\right)\right] \subseteq \operatorname{Arc}\left(1, \lambda_{k}\right)$ and $\operatorname{Arg}\left(R_{k}\left(R_{k}\left(\xi_{n_{1}}\right)\right)\right)>\operatorname{Arg}\left(R_{k}(\xi)\right)$.

(b) For all $z \in \operatorname{Arc}\left[R_{k}\left(\xi_{n_{1}}\right), R_{k}\left(\xi_{n_{2}}\right)\right]$ we have ||$f_{k}^{\prime}(z)|-\alpha|<\delta$.

(c) For all $z_{1}, z_{2} \in \operatorname{Arc}\left[R_{k}\left(\xi_{n_{1}}\right), R_{k}\left(\xi_{n_{2}}\right)\right]$ we have ||$R_{k}^{\prime}\left(z_{1}\right) / R_{k}^{\prime}\left(z_{2}\right)|-1|<\epsilon_{2}$.

That it is possible to choose $n_{1}, n_{2}$ such that the first two properties hold follows from the fact that both $R_{k}$ and the derivative of $f_{k}$ are continuous on $\operatorname{Arc}\left[\overline{\lambda_{k}}, \lambda_{k}\right]$. The existence of $n_{1}, n_{2}$ satisfying the third property follows from the fact that the derivative of $z \mapsto R_{k}(z)$ is continuous and nonzero on $\operatorname{Arc}\left(1, \lambda_{k}\right)$.

Let $g_{0}=f_{\xi_{n_{1}}, k}$ and $g_{1}=f_{\xi_{n_{2}}, k}$. Since $\xi_{n_{1}}, \xi_{n_{2}}$ are implemented by rooted trees in $\mathcal{T}_{d+1}$ with root degrees at most $m$, we have by Lemma 4 that, if $r$ is implemented by a rooted tree in $\mathcal{T}_{d+1}$, then $g_{i}(r)$ is the field implemented by a tree in $\mathcal{T}_{d+1}$ and root degree $m+k \leq d$. Let $A=\operatorname{Arc}\left[R_{k}\left(\xi_{n_{1}}\right), R_{k}\left(\xi_{n_{2}}\right)\right]$ and note that the maps $g_{0}, g_{1}$ have the respective endpoints of $A$ as fixed points. Furthermore, ||$g_{i}^{\prime}(z)|-\alpha|<\delta$ for all $z \in A$ and thus it follows from Corollary 29 that there is a triple $\omega_{1}, \omega_{2}, \omega_{3} \in \Omega$ such that for all triples $p_{i} \in g_{\omega_{i}}(A)$ we have $\operatorname{Arg}\left(p_{1}\right)<\operatorname{Arg}\left(p_{2}\right)<\operatorname{Arg}\left(p_{3}\right)$ and

$$
\left|\frac{\ell\left(\operatorname{Arc}\left[p_{1}, p_{2}\right]\right)}{\ell\left(\operatorname{Arc}\left[p_{2}, p_{3}\right]\right)}-1\right|<\epsilon_{1}
$$


The orbit of $\xi_{n_{2}}$ under iteration of $g_{1}$ converges to $R_{k}\left(\xi_{n_{2}}\right)$ approaching from an anti-clockwise direction and thus there is some number $N$ such that if we let $\omega_{N}$ be the constant 1 sequence of length $N$, $g_{\omega_{N}}\left(\xi_{n_{2}}\right) \in A$. For $i=1,2,3$ we define $\zeta_{i}=g_{\omega_{i} \oplus \omega_{N}}\left(\xi_{n_{2}}\right)$ and note that each $\zeta_{i}$ is contained in the interval $\left(\overline{\lambda_{k}}, \lambda_{k}\right)$ and is implemented by a rooted tree in $\mathcal{T}_{d+1}$ with root degree $m+k$. Furthermore, we have $\operatorname{Arg}\left(\zeta_{1}\right)<\operatorname{Arg}\left(\zeta_{2}\right)<\operatorname{Arg}\left(\zeta_{3}\right)$ and

$$
\left|\frac{\ell\left(\operatorname{Arc}\left[\zeta_{1}, \zeta_{2}\right]\right)}{\ell\left(\operatorname{Arc}\left[\zeta_{2}, \zeta_{3}\right]\right)}-1\right|<\epsilon_{1} .
$$

Let $h_{i}=f_{\zeta_{i}, k}$. Analogous to the above, if $r$ is implemented by a rooted tree in $\mathcal{T}_{d+1}$, then $h_{i}(r)$ is implemented by a rooted tree in $\mathcal{T}_{d+1}$ with root degree at most $m+2 k \leq d$. Redefine $A=\operatorname{Arc}\left[R_{k}\left(\zeta_{1}\right), R_{k}\left(\zeta_{3}\right)\right]$. We will show that we can choose $\epsilon_{1}$ and $\epsilon_{2}$ sufficiently small such that $A=h_{1}(A) \cup h_{2}(A) \cup h_{3}(A)$. To do this, define $A_{1}=\operatorname{Arc}\left[R_{k}\left(\zeta_{1}\right), R_{k}\left(\zeta_{2}\right)\right]$ and $A_{2}=\operatorname{Arc}\left[R_{k}\left(\zeta_{2}\right), R_{k}\left(\zeta_{3}\right)\right]$. It follows from the mean value theorem that there are $x_{i} \in \operatorname{Arc}\left[\zeta_{i}, \zeta_{i+1}\right]$ such that $\ell\left(A_{i}\right)=\left|R_{k}^{\prime}\left(x_{i}\right)\right| \cdot \ell\left(\operatorname{Arc}\left[\zeta_{i}, \zeta_{i+1}\right]\right)$ for $i=1,2$. Because both $x_{1}$ and $x_{2}$ lie in $\operatorname{Arc}\left[R_{k}\left(\xi_{n_{1}}\right), R_{k}\left(\xi_{n_{2}}\right)\right]$, it follows from property (c) above that we can write $\left|R_{k}^{\prime}\left(x_{1}\right) / R_{k}^{\prime}\left(x_{2}\right)\right|=1+r_{2}$ for some $r_{2} \in \mathbb{R}$ with $\left|r_{2}\right|<\epsilon_{2}$. We use the bound in (14) to obtain the following inequality:

$$
\begin{aligned}
\left|\frac{\ell\left(A_{1}\right)}{\ell\left(A_{2}\right)}-1\right| & =\left|\frac{\left|R_{k}^{\prime}\left(x_{1}\right)\right| \cdot \ell\left(\operatorname{Arc}\left[\zeta_{1}, \zeta_{2}\right]\right)}{\left|R_{k}^{\prime}\left(x_{2}\right)\right| \cdot \ell\left(\operatorname{Arc}\left[\zeta_{2}, \zeta_{3}\right]\right)}-1\right|=\left|\left(1+r_{2}\right) \frac{\ell\left(\operatorname{Arc}\left[\zeta_{1}, \zeta_{2}\right]\right)}{\ell\left(\operatorname{Arc}\left[\zeta_{2}, \zeta_{3}\right]\right)}-1\right| \\
& \leq\left|1+r_{2}\right| \cdot\left|\frac{\ell\left(\operatorname{Arc}\left[\zeta_{1}, \zeta_{2}\right]\right)}{\ell\left(\operatorname{Arc}\left[\zeta_{2}, \zeta_{3}\right]\right)}-1\right|+\left|r_{2}\right|<\left|1+r_{2}\right| \cdot \epsilon_{1}+\left|r_{2}\right| \\
& \leq \epsilon_{1}+\epsilon_{2}+\epsilon_{1} \cdot \epsilon_{2} .
\end{aligned}
$$

Let $\epsilon_{3}=\epsilon_{1}+\epsilon_{2}+\epsilon_{1} \cdot \epsilon_{2}$ and note that $\epsilon_{3}$ can be made arbitrarily small by choosing $\epsilon_{1}$ and $\epsilon_{2}$ sufficiently small. It follows that there is some $r_{3} \in \mathbb{R}$ with $\left|r_{3}\right|<\epsilon_{3}$ such that $\ell\left(A_{1}\right)=\left(1+r_{3}\right) \cdot \ell\left(A_{2}\right)$. Because $\operatorname{Arg}\left(R_{k}\left(\zeta_{1}\right)\right)>\operatorname{Arg}\left(R_{k}\left(R_{k}\left(\xi_{n_{1}}\right)\right)\right)>\operatorname{Arg}\left(R_{k}(\xi)\right)$, we find that $1>\left|f_{k}^{\prime}(z)\right|>\alpha$ for all $z \in A$ and thus $1>\left|h_{i}^{\prime}(z)\right|>\alpha$ for all $z \in A$ and $i=1,2,3$. It follows that

$$
\begin{gathered}
\ell\left(h_{1}\left(A_{1} \cup A_{2}\right)\right)+\ell\left(h_{2}\left(A_{1}\right)\right)>\alpha \cdot\left(\ell\left(A_{1}\right)+\ell\left(A_{2}\right)\right)+\alpha \cdot \ell\left(A_{1}\right)=\alpha \cdot\left(2 \ell\left(A_{1}\right)+\ell\left(A_{2}\right)\right) \\
=\alpha \cdot\left(2+\frac{1}{1+r_{3}}\right) \ell\left(A_{1}\right)=\alpha \cdot \frac{3+2 r_{3}}{1+r_{3}} \cdot \ell\left(A_{1}\right)
\end{gathered}
$$

and

$$
\begin{aligned}
\ell\left(h_{2}\left(A_{2}\right)\right)+\ell\left(h_{3}\left(A_{1} \cup A_{2}\right)\right) & >\alpha \cdot \ell\left(A_{2}\right)+\alpha \cdot\left(\ell\left(A_{1}\right)+\ell\left(A_{2}\right)\right)=\alpha \cdot\left(\ell\left(A_{1}\right)+2 \ell\left(A_{2}\right)\right) \\
& =\alpha \cdot\left(\left(1+r_{3}\right)+2\right) \ell\left(A_{2}\right)=\alpha \cdot\left(3+r_{3}\right) \cdot \ell\left(A_{2}\right) .
\end{aligned}
$$

Because $\alpha>1 / 3$, we can choose $\epsilon_{3}$ sufficiently small such that

$$
\ell\left(h_{1}\left(A_{1} \cup A_{2}\right)\right)+\ell\left(h_{2}\left(A_{1}\right)\right)>\ell\left(A_{1}\right) \quad \text { and } \quad \ell\left(h_{2}\left(A_{2}\right)\right)+\ell\left(h_{3}\left(A_{1} \cup A_{2}\right)\right)>\ell\left(A_{2}\right) .
$$

Because $h_{1}\left(A_{1} \cup A_{2}\right)$ and $h_{2}\left(A_{1}\right)$ share the respective endpoints of $A_{1}$, it follows that $A_{1} \subseteq h_{1}\left(A_{1} \cup A_{2}\right) \cup$ $h_{2}\left(A_{1}\right)$. Similarly, we find that $A_{2} \subseteq h_{3}\left(A_{1} \cup A_{2}\right) \cup h_{2}\left(A_{2}\right)$. It follows that $A=h_{1}(A) \cup h_{2}(A) \cup h_{3}(A)$. Finally, let $s=h_{3}^{N}(1)$, where we have taken $N$ sufficiently large such that $s \in A$, and consider

$$
\mathcal{S}=\left\{\left(h_{i_{1}} \circ \cdots \circ h_{i_{l}}\right)(s): l \in \mathbb{Z}_{\geq 1} \text { and } i_{1}, \ldots, i_{l} \in\{1,2,3\}\right\}
$$

It follows from Corollary 20 that $\mathcal{S}$ is a dense subset of $A$. Every $r \in \mathcal{S}$ is implemented by a rooted tree in $\mathcal{T}_{d+1}$ with root degree $m+2 k \leq d$. Finally, we let

$$
\mathcal{S}_{2}=\left\{f_{\lambda, d}^{n}(r): r \in \mathcal{S}, n \geq 1\right\}
$$


and we find, because $\left|f_{d}^{\prime}(z)\right|>1$ for all $z \in \mathbb{S}-\{1\}$, that $\mathcal{S}_{2}$ is dense in $\mathbb{S}$. Every $r \in \mathcal{S}_{2}$ is implemented by a tree in $\mathcal{T}_{d+1}$. This shows that in this case item (1) of the lemma holds.

Lemma 31. Suppose $d \in \mathbb{Z}_{\geq 5}, b \in\left(0, \frac{d-1}{d+1}\right] \cap \mathbb{Q}, \lambda \in \mathbb{S}_{\mathbb{Q}} \backslash\{ \pm 1\}$ and $\xi \in \Lambda_{3}(b) \cap \mathbb{S}_{\mathbb{Q}}$ with $\xi \neq \pm 1$.

Suppose there is a rooted tree in $\mathcal{T}_{d+1}$ with root degree at most $d-5$ and field $\xi$. Then the set of fields implemented by rooted trees in $\mathcal{T}_{d+1}$ is dense in $\mathbb{S}$.

Proof. It follows from Lemma 16 that there is $\sigma \in \mathbb{S}$ with $\left|f_{3}^{\prime}(\sigma)\right|>1$ together with a sequence $\left\{\zeta_{n}\right\}_{n \geq 1}$ accumulating on $\sigma$ such that every $\zeta_{n}$ is the field implemented by a tree in $\mathcal{T}_{d+1}$ whose root degree is bounded by $(d-5)+3=d-2$. We can now apply Lemma 30 with $k=1$. It follows that either the set of fields implemented by rooted trees in $\mathcal{T}_{d+1}$ is dense in $\mathbb{S}$ or there is a tree in $\mathcal{T}_{d+1}$ with root degree at most $(d-2)+1=d-1$ and field $\zeta \in \operatorname{Arc}\left(\lambda_{1}, \overline{\lambda_{1}}\right) \backslash\{-1\}$. We conclude from Lemma 14 that $f_{\zeta, 1}$ is conjugate to an irrational rotation and thus the orbit $\left\{f_{\zeta, 1}^{n}(1)\right\}_{n \geq 1}$ is dense in $\mathbb{S}$. Every element in this orbit is implemented by a rooted tree in $\mathcal{T}_{d+1}$, and thus we are done.

\section{Proof of Lemma 17}

We are now ready to prove Lemma 17, which we restate here for convenience.

Lemma 17 . Let $k, d \in \mathbb{Z}_{\geq 2}$ with $k \leq d, b \in\left(\frac{d-2}{d}, \frac{d-1}{d+1}\right] \cap \mathbb{Q}$ and $\lambda \in \mathbb{S}_{\mathbb{Q}} \backslash\{ \pm 1\}$. Suppose there exists $a$ rooted tree in $\mathcal{T}_{d+1}$ with root degree at most $d-k$ that implements a field $\xi \neq 1$ with the property that $\left|f_{k}^{\prime}(\xi)\right| \geq 1$ and $\xi \in \operatorname{Arc}\left[\overline{\lambda_{\lfloor k / 2\rfloor}}, \lambda_{\lfloor k / 2\rfloor}\right]$. Then the set of fields implemented by trees in $\mathcal{T}_{d+1}$ is dense in $\mathbb{S}$.

Proof. The proof consists of a careful case analysis. We give a seperate argument first for when $k$ is a power of two and for when $k+1$ is a power of 2 and then for each value of $k$ within the set $\{5,6,9,10,11,12,13,14,17\}$ and, lastly, we prove the statement for all other $k$.

We remark that in some cases we will show that the set of fields implemented by rooted trees in $\mathcal{T}_{d+1}$ is dense in an $\operatorname{arc} A$ of the circle. Since $b$ is such that $\left|f_{d}^{\prime}(z)\right|>1$ for all $z \in \mathbb{S} \backslash\{1\}$ (see (2) of Lemma 10 ), it follows that for all $\operatorname{arcs} A$ there is an $N \geq 1$ such that $f_{\lambda, d}^{N}(A)=\mathbb{S}$. Density of fields in the whole unit circle therefore follows from density in $A$.

First suppose $k=2^{m}$ is a power of 2 . In this case, $\xi \in \operatorname{Arc}\left[\overline{\lambda_{2^{m-1}}}, \lambda_{2^{m-1}}\right] \backslash\{1\}$ is implemented by a rooted tree in $\mathcal{T}_{d+1}$ with root degree at most $d-2^{m}$ and with $\left|f_{2^{m}}^{\prime}(\xi)\right| \geq 1$. Let $\xi_{2}=f_{\xi, 1}(\xi)$. By item (v) of Lemma 10, we have $\xi \in \operatorname{Arc}\left[\overline{\lambda_{1}}, \lambda_{1}\right] \backslash\{1\}$ and hence $\xi_{2} \neq \xi$ by item (iv) of the same lemma. Moreover, by Lemma $4, \xi_{2}$ is the field of a rooted tree in $\mathcal{T}_{d+1}$ with root degree at most $d-\left(2^{m}-1\right)$. If $\xi_{2} \in \operatorname{Arc}\left(\lambda_{2^{m-1}}, \overline{\lambda_{2^{m-1}}}\right)$, then the desired result follows from Lemma 25 . Otherwise, $\xi, \xi_{2} \in \operatorname{Arc}\left[\overline{\lambda_{2^{m-1}}}, \lambda_{2^{m-1}}\right]$ and the result follows from applying Lemma 22 to these two parameters.

Now suppose $k+1$ is a power of 2 , so $k=2^{m+1}-1$ for $m \geq 1$. In this case, $\xi \in \operatorname{Arc}\left[\overline{\lambda_{2^{m}-1}}, \lambda_{2^{m}-1}\right] \backslash\{1\}$ is implemented by a rooted tree in $\mathcal{T}_{d+1}$ with root degree at most $d-\left(2^{m+1}-1\right)$ and with $\left|f_{2^{m+1}-1}^{\prime}(\xi)\right| \geq 1$. If $\xi \in \operatorname{Arc}\left(\lambda_{2^{m}}, \overline{\lambda_{2^{m}}}\right)$, the result follows from Lemma 25. Otherwise, if $\xi \in \operatorname{Arc}\left[\overline{\lambda_{2^{m}}}, \lambda_{2^{m}}\right]$, the result follows from Lemma 21.

We now continue with the list of individual cases.

$\mathbf{k}=\mathbf{5}:$ In this case, $\xi \in \operatorname{Arc}\left[\overline{\lambda_{2}}, \lambda_{2}\right] \backslash\{1\}$ is the field of a rooted tree with root degree at most $d-5$ and with $\left|f_{5}^{\prime}(\xi)\right| \geq 1$. If $\xi \in \operatorname{Arc}\left(\lambda_{3}, \overline{\lambda_{3}}\right)$, the result follows from Lemma 31. Otherwise, if $\xi \in \operatorname{Arc}\left[\overline{\lambda_{3}}, \lambda_{3}\right]$, the result follows from Lemma 21 .

$\mathbf{k}=\mathbf{6}:$ In this case, $\xi \in \operatorname{Arc}\left[\overline{\lambda_{3}}, \lambda_{3}\right] \backslash\{1\}$ is implemented by a rooted tree in $\mathcal{T}_{d+1}$ with root degree at most $d-6$ and with $\left|f_{6}^{\prime}(\xi)\right| \geq 1$. Let $\xi_{2}=f_{\xi, 1}(\xi)$, which is the field of a rooted tree in $\mathcal{T}_{d+1}$ with root degree at most $d-5$. If $\xi_{2} \in \operatorname{Arc}\left(\lambda_{3}, \overline{\lambda_{3}}\right)$, then the result follows from Lemma 31. Otherwise, $\xi, \xi_{2} \in \operatorname{Arc}\left[\overline{\lambda_{3}}, \lambda_{3}\right]$ and the result follows from applying Lemma 22 to these two parameters.

$\mathbf{k}=9$ : In this case, $\xi \in \operatorname{Arc}\left[\overline{\lambda_{4}}, \lambda_{4}\right] \backslash\{1\}$ is implemented by a rooted tree in $\mathcal{T}_{d+1}$ with root degree at most $d-9$ and with $\left|f_{9}^{\prime}(\xi)\right| \geq 1$. Consider the orbit $\left\{f_{\xi, 1}^{n}(\xi): n \geq 1\right\}$. The elements of this orbit are implemented by trees in $\mathcal{T}_{d+1}$ with root degree at most $d-8$ and they accumulate on $R_{1}(\xi)$. Note that $\left|f_{9}^{\prime}\left(R_{1}(\xi)\right)\right|>1$. It follows from Lemma 30 that either we obtain the desired density or we obtain a 
rooted tree with root degree at most $(d-8)+3=d-5$ that implements a field in $\operatorname{Arc}\left(\lambda_{3}, \overline{\lambda_{3}}\right)$. In this latter case, the result follows from applying Lemma 31 to this tree.

$\mathbf{k}=\mathbf{1 0}:$ In this case, $\xi \in \operatorname{Arc}\left[\overline{\lambda_{5}}, \lambda_{5}\right] \backslash\{1\}$ is implemented by a rooted tree in $\mathcal{T}_{d+1}$ with root degree at most $d-10$ and with $\left|f_{10}^{\prime}(\xi)\right| \geq 1$. Then it follows from Lemma 23 that the orbit of 1 under the action of the semigroup generated by $f_{\xi, 3}, f_{\xi, 4}$ and $f_{\xi, 5}$ is dense in an arc of $\mathbb{S}$, in which case the result follows. Or we can conclude that $\left|f_{5}^{\prime}\left(R_{5}(\xi)\right)\right|>\frac{43}{50}$. In that case, we consider the orbit $\mathcal{R}=\left\{f_{\xi, 5}^{n}(\xi): n \geq 1\right\}$. This orbit accumulates on $R_{5}(\xi)$ and every element is implemented by a rooted tree with root degree at most $d-10+5=d-5$. If $R_{5}(\xi) \in \operatorname{Arc}\left(\lambda_{3}, \overline{\lambda_{3}}\right)$, then there are also fields $\zeta \in \mathcal{R}$ with $\zeta \in \operatorname{Arc}\left(\lambda_{3}, \overline{\lambda_{3}}\right)$. In that case, we can apply Lemma 31 to obtain density of the fields. Otherwise, if $R_{5}(\xi) \in \operatorname{Arc}\left[\overline{\lambda_{3}}, \lambda_{3}\right]$, then we can find $\zeta_{1}, \zeta_{2} \in \mathcal{R}$ such that $\zeta_{1}, \zeta_{2} \in \operatorname{Arc}\left[\overline{\lambda_{3}}, \lambda_{3}\right]$ are distinct, lie in the same half-plane and $\left|f_{5}^{\prime}\left(\zeta_{i}\right)\right|>\frac{43}{50}$ for $i=1,2$. It follows that for both fields $\zeta_{i}$ we have

$$
\left|f_{6}^{\prime}\left(R_{3}\left(\zeta_{i}\right)\right)\right|>\left|f_{6}^{\prime}\left(\zeta_{i}\right)\right|=\frac{6}{5} \cdot\left|f_{5}^{\prime}\left(\zeta_{i}\right)\right|>\frac{6}{5} \cdot \frac{43}{50}=\frac{129}{125}>1 .
$$

Density of the fields now follows from applying Lemma 22 to $\zeta_{1}$ and $\zeta_{2}$.

$\mathbf{k}=11:$ In this case, $\xi \in \operatorname{Arc}\left[\overline{\lambda_{5}}, \lambda_{5}\right] \backslash\{1\}$ is implemented by a rooted tree in $\mathcal{T}_{d+1}$ with root degree at most $d-11$ and with $\left|f_{11}^{\prime}(\xi)\right| \geq 1$. If $\xi \in \operatorname{Arc}\left[\overline{\lambda_{6}}, \lambda_{6}\right]$, the result follows from Lemma 21 . Otherwise, if $\xi \in \operatorname{Arc}\left(\lambda_{6}, \overline{\lambda_{6}}\right)$, we apply Lemma 16 to find a parameter $\sigma \in \mathbb{S}$ with $\left|f_{6}^{\prime}(\sigma)\right|>1$ together with a sequence of fields $\left\{\zeta_{n}\right\}_{n \geq 1}$ accumulating on $\sigma$ such that every $\zeta_{n}$ is implemented by a rooted tree in $\mathcal{T}_{d+1}$ whose root degree is at most $d-11+6=d-5$. If there is any $\zeta_{n} \in \operatorname{Arc}\left(\lambda_{3}, \overline{\lambda_{3}}\right)$, then density of the fields in the circle follows from Lemma 31. Otherwise, the sequence accumulates on $\sigma$ from inside $\operatorname{Arc}\left[\overline{\lambda_{3}}, \lambda_{3}\right]$ and thus we can find $\zeta_{n_{1}}, \zeta_{n_{2}} \in \operatorname{Arc}\left[\overline{\lambda_{3}}, \lambda_{3}\right]$ that are distinct, lie in the same half-plane and have the property that $\left|f_{6}^{\prime}\left(\zeta_{i}\right)\right|>1$ for $i=1,2$. The desired density now follows from applying Lemma 22 to $\zeta_{1}$ and $\zeta_{2}$.

$\mathbf{k}=12:$ In this case, $\xi \in \operatorname{Arc}\left[\overline{\lambda_{6}}, \lambda_{6}\right] \backslash\{1\}$ is implemented by a rooted tree in $\mathcal{T}_{d+1}$ with root degree at most $d-12$ and with $\left|f_{12}^{\prime}(\xi)\right| \geq 1$. This case can be done in a very similar way to the $k=9$ case. Consider the orbit $\left\{f_{\xi, 1}^{n}(\xi): n \geq 1\right\}$. The elements of this orbit are fields of trees in $\mathcal{T}_{d+1}$ with root degree at most $d-11$ and they accumulate on $R_{1}(\xi)$. Note that $\left|f_{12}^{\prime}\left(R_{1}(\xi)\right)\right|>1$. It follows from Lemma 30 that either we obtain the desired density or we obtain a rooted tree with root degree at most $(d-11)+4=d-7$ and field in $\operatorname{Arc}\left(\lambda_{4}, \overline{\lambda_{4}}\right)$. In this latter case, the result follows from applying Lemma 25 to this tree.

$\mathbf{k}=13:$ In this case, $\xi \in \operatorname{Arc}\left[\overline{\lambda_{6}}, \lambda_{6}\right] \backslash\{1\}$ is implemented by a rooted tree in $\mathcal{T}_{d+1}$ with root degree at most $d-13$ and with $\left|f_{13}^{\prime}(\xi)\right| \geq 1$. Then it follows from Lemma 23 that the orbit of 1 under the action of the semigroup generated by $f_{\xi, 4}, f_{\xi, 5}$ and $f_{\xi, 6}$ is dense in an arc of $\mathbb{S}$, in which case the result follows. Or we can conclude that $\left|f_{6}^{\prime}\left(R_{6}(\xi)\right)\right|>\frac{10}{13}$. In that case, we consider the orbit $\mathcal{R}=\left\{f_{\xi, 6}^{n}(\xi): n \geq 1\right\}$. This orbit accumulates on $R_{6}(\xi)$ and every element is implemented by a rooted tree in $\mathcal{T}_{d+1}$ with root degree at most $d-13+6=d-7$. If $R_{6}(\xi) \in \operatorname{Arc}\left(\lambda_{4}, \overline{\lambda_{4}}\right)$, then there is also a field $\zeta \in \mathcal{R}$ with $\zeta \in \operatorname{Arc}\left(\lambda_{4}, \overline{\lambda_{4}}\right)$. In that case, we can apply Lemma 25 to obtain density of the fields. Otherwise, if $R_{6}(\xi) \in \operatorname{Arc}\left[\overline{\lambda_{4}}, \lambda_{4}\right]$, then we can find $\zeta_{1}, \zeta_{2} \in \mathcal{R}$ such that $\zeta_{1}, \zeta_{2} \in \operatorname{Arc}\left[\overline{\lambda_{4}}, \lambda_{4}\right]$ are distinct, lie in the same half-plane and $\left|f_{6}^{\prime}\left(\zeta_{i}\right)\right|>\frac{10}{13}$ for $i=1,2$. It follows that for both fields $\zeta_{i}$ we have

$$
\left|f_{8}^{\prime}\left(R_{4}\left(\zeta_{i}\right)\right)\right|>\left|f_{8}^{\prime}\left(\zeta_{i}\right)\right|=\frac{8}{6} \cdot\left|f_{6}^{\prime}\left(\zeta_{i}\right)\right|>\frac{8}{6} \cdot \frac{10}{13}=\frac{40}{39}>1 .
$$

Density of the fields now follows from applying Lemma 22 to $\zeta_{1}$ and $\zeta_{2}$.

$\mathbf{k}=14:$ In this case, $\xi \in \operatorname{Arc}\left[\overline{\lambda_{7}}, \lambda_{7}\right] \backslash\{1\}$ is implemented by a rooted tree in $\mathcal{T}_{d+1}$ with root degree at most $d-14$ and with $\left|f_{14}^{\prime}(\xi)\right| \geq 1$. Then it follows from Lemma 23 that the orbit of 1 under the action of the semigroup generated by $f_{\xi, 5}, f_{\xi, 6}$ and $f_{\xi, 7}$ is dense in an arc of $\mathbb{S}$, in which case the result follows. Or we can conclude that $\left|f_{7}^{\prime}\left(R_{7}(\xi)\right)\right|>\frac{89}{98}$. In that case, we consider the orbit $\mathcal{R}=\left\{f_{\xi, 7}^{n}(\xi): n \geq 1\right\}$. This orbit accumulates on $R_{7}(\xi)$ and every element is implemented by a rooted tree in $\mathcal{T}_{d+1}$ with root degree at most $d-14+7=d-7$. If $R_{7}(\xi) \in \operatorname{Arc}\left(\lambda_{4}, \overline{\lambda_{4}}\right)$, then there is also a field $\zeta \in \mathcal{R}$ with $\zeta \in \operatorname{Arc}\left(\lambda_{4}, \overline{\lambda_{4}}\right)$. In that case, we can apply Lemma 25 to obtain density of the fields. Otherwise, if 
$R_{7}(\xi) \in \operatorname{Arc}\left[\overline{\lambda_{4}}, \lambda_{4}\right]$, then we can find $\zeta_{1}, \zeta_{2} \in \mathcal{R}$ such that $\zeta_{1}, \zeta_{2} \in \operatorname{Arc}\left[\overline{\lambda_{4}}, \lambda_{4}\right]$ are distinct, lie in the same half-plane and $\left|f_{7}^{\prime}\left(\zeta_{i}\right)\right|>\frac{89}{98}$ for $i=1,2$. It follows that for both fields $\zeta_{i}$ we have

$$
\left|f_{8}^{\prime}\left(R_{4}\left(\zeta_{i}\right)\right)\right|>\left|f_{8}^{\prime}\left(\zeta_{i}\right)\right|=\frac{8}{7} \cdot\left|f_{7}^{\prime}\left(\zeta_{i}\right)\right|>\frac{8}{7} \cdot \frac{89}{98}=\frac{356}{343}>1 .
$$

Density of the fields now follows from applying Lemma 22 to $\zeta_{1}$ and $\zeta_{2}$.

$\mathbf{k}=17:$ In this case, $\xi \in \operatorname{Arc}\left[\overline{\lambda_{8}}, \lambda_{8}\right] \backslash\{1\}$ is implemented by a rooted tree in $\mathcal{T}_{d+1}$ with root degree at most $d-17$ and with $\left|f_{17}^{\prime}(\xi)\right| \geq 1$. If $\xi \in \operatorname{Arc}\left[\overline{\lambda_{9}}, \lambda_{9}\right]$, the result follows from Lemma 21; therefore, we assume that $\xi \in \operatorname{Arc}\left(\lambda_{9}, \overline{\lambda_{9}}\right)$. We apply Lemma 16 to find a parameter $\sigma \in \mathbb{S}$ with $\left|f_{9}^{\prime}(\sigma)\right|>1$ together with a sequence of fields $\left\{\zeta_{n}\right\}_{n \geq 1}$ accumulating on $\sigma$ such that every $\zeta_{n}$ is implemented by a rooted tree in $\mathcal{T}_{d+1}$ whose root degree is at most $d-17+9=d-8$. It follows from Lemma 30 that we either obtain the required density of fields or there is a tree in $\mathcal{T}_{d+1}$ whose root degree is bounded by $d-5$ with field inside $\operatorname{Arc}\left(\lambda_{3}, \overline{\lambda_{3}}\right)$. In the latter case, the result follows from Lemma 31.

Finally, we complete the proof for $k>17$. In that case, write $k=2 m$ if $k$ is even and $k=2 m+1$ if $k$ is odd. Note that $m \geq 9$. We are then given that $\xi \in \operatorname{Arc}\left[\overline{\lambda_{m}}, \lambda_{m}\right] \backslash\{1\}$ is implemented by a rooted tree in $\mathcal{T}_{d+1}$ with root degree at most $d-k$ and with $\left|f_{k}^{\prime}(\xi)\right| \geq 1$. It follows from Corollary 24 that the orbit of 1 under the action of the semigroup generated by $f_{\xi, m-3}, f_{\xi, m-2}, f_{\xi, m-1}$ and $f_{\xi, m}$ is dense in an arc of $\mathbb{S}$ from which our desired conclusion follows. This finishes the proof of Lemma 17.

\section{Fast implementation of fields}

In this section, we bootstrap Theorem 6 to obtain fast algorithms for implementing fields which will be important in our reductions. For a number $\alpha=p / q \in \mathbb{Q}$ with $\operatorname{gcd}(p, q)=1$, we use $\operatorname{size}(\alpha)$ to denote the total number of bits needed to represent $p, q$, and we extend this to numbers in $\mathbb{C}_{\mathbb{Q}}$ by adding the sizes of the real and imaginary parts. For $\alpha_{1}, \ldots, \alpha_{t} \in \mathbb{C}_{\mathbb{Q}}$, we denote by $\operatorname{size}\left(\alpha_{1}, \ldots, \alpha_{t}\right)$ the total of the sizes of $\alpha_{1}, \ldots, \alpha_{t}$.

Lemma 32. Fix an integer $\Delta \geq 3$, a rational number $b \in(0,1)$ and $\lambda \in \mathbb{S}_{\mathbb{Q}}(\Delta-1, b)$. Then, there is an algorithm, which on input $\hat{\lambda} \in \mathbb{S}_{\mathbb{Q}}$ and rational $\epsilon>0$, returns in time poly $\left.(\operatorname{size}(\hat{\lambda}, \epsilon))\right)$ a rooted tree $T$ in $\mathcal{T}_{\Delta}$ with root degree 1 that implements a field $\lambda^{\prime}$ such that $\left|\lambda^{\prime}-\hat{\lambda}\right| \leq \epsilon$.

Proof of Lemma 32. Let $d=\Delta-1$. We start by setting up some parameters that will be useful.

Let $\lambda_{1}$ be as in Lemma 10. As $\tilde{\lambda}$ approaches $\lambda_{1}$ from inside $\operatorname{Arc}\left(1, \lambda_{1}\right)$, we know that $R_{1}(\tilde{\lambda})$ approaches $R_{1}\left(\lambda_{1}\right)$. Since $\left|f_{1}^{\prime}\left(R_{1}\left(\lambda_{1}\right)\right)\right|=1$, there must be $\tilde{\lambda} \in \operatorname{Arc}\left(1, \lambda_{1}\right)$ such that $\left|f_{1}^{\prime}\left(R_{1}(\xi)\right)\right| \in\left(\frac{1}{2}, 1\right)$ for all $\xi \in \operatorname{Arc}\left(\tilde{\lambda}, \lambda_{1}\right)$. By Theorem 6 , there exist trees $T_{1}, T_{2}$ in $\mathcal{T}_{d+1}$ with root degree 1 and fields $\xi_{1}, \xi_{2} \in \operatorname{Arc}\left(\tilde{\lambda}, \lambda_{1}\right) \cap \mathbb{S}_{\mathbb{Q}}$ with $\operatorname{Arg}\left(\xi_{1}\right)<\operatorname{Arg}\left(\xi_{2}\right)$. Because the map $\xi \mapsto R_{1}(\xi)$ is orientation preserving with nonzero derivative, we have $\operatorname{Arg}\left(R_{1}\left(\xi_{1}\right)\right)<\operatorname{Arg}\left(R_{1}\left(\xi_{2}\right)\right)$. For $i \in\{1,2\}$, the fixed point $R_{1}\left(\xi_{i}\right)$ is a solution to the quadratic equation $\xi_{i}(z+b)=z(b z+1)$ and hence we can approximate it with any desired rational precision $\tau>0$ in time poly $(\operatorname{size}(\tau))$.

Let $I=\operatorname{Arc}\left(R_{1}\left(\xi_{1}\right), R_{1}\left(\xi_{2}\right)\right)$ and note that this arc is contained in the upper half-plane. We will show that the arc $I$ gets mapped onto $\mathbb{S}$ in a fixed number of applications of $f_{\lambda, d}$. The idea of the algorithm is then to find a small enough neighbourhood of a point in $I$ that gets mapped close to the field that we are trying to (approximately) implement. Then we use that we are able to quickly and accurately approach any value inside $I$ using $f_{\xi_{1}, 1}$ and $f_{\xi_{2}, 1}$. This algorithm is very similar to the proof of Lemma 19.

We now show that $I$ gets mapped onto $\mathbb{S}$ in a fixed number of applications of $f_{\lambda, d}$. We first consider the case that $b \in\left(0, \frac{d-1}{d+1}\right]$. Let $C_{1}=\left|f_{\lambda, d}^{\prime}(1)\right|=d \frac{1-b}{1+b}$ and let $C_{2}=\left|f_{\lambda, d}^{\prime}(-1)\right|=d \frac{1+b}{1-b}$. Note that $C_{1}$ and $C_{2}$ are both greater than 1 and that for any $z \in \mathbb{S}$ the inequality $C_{1} \leq\left|f_{\lambda, d}^{\prime}(z)\right| \leq C_{2}$ holds (cf. item (i) of Lemma 10). This means that for any circular arc $J$ and integer $n$ we get

$$
C_{1}^{n} \cdot \ell(J) \leq \ell\left(f_{\lambda, d}^{n}(J)\right) \leq C_{2}^{n} \cdot \ell(J) .
$$

From this, we deduce that $f_{\lambda, d}^{N}(I)=\mathbb{S}$, where $N=\left\lceil\frac{\log (2 \pi / \ell(I))}{\log \left(C_{1}\right)}\right\rceil$. 
Next, in case $b \in\left(\frac{d-1}{d+1}, 1\right)$, we recall the conformal metric $\mu$ from the proof of Lemma 15 . Let us denote the length of a circular arc $J$ with respect to this metric by length $(J)$ and denote $c=\operatorname{length}(\mathbb{S})$. Since there exists a constant $\kappa>1$ such that $f_{d, \lambda}$ is uniformly expanding on $\mathbb{S}$ with a factor $\kappa$ with respect to this metric, it follows that $f_{\lambda, d}^{N}(I)=\mathbb{S}$, where $N=\left\lceil\frac{\log (c / \operatorname{length}(I))}{\log (\kappa)}\right\rceil$. Note that the right-hand side of (15) is also valid for $b \in\left(\frac{d-1}{d+1}, 1\right)$ (with $C_{2}$ defined in the same way).

Let $x_{0}, \ldots, x_{m}$ be points such that the clockwise arcs between $x_{i-1}$ and $x_{i}$ form a partition of $I$ with $x_{0}=R_{1}\left(\xi_{1}\right), x_{m}=R_{1}\left(\xi_{2}\right)$ and chosen so that $x_{1}, \ldots, x_{m-1} \in \mathbb{S}_{\mathbb{Q}}$ and the length of an arc between two subsequent points is less than $2 \pi / C_{2}^{N}$. In this way, we ensure that these arcs are not mapped onto the whole circle by $N$ applications of $f_{\lambda, d}$ and thus each arc is bijectively mapped to an arc on the unit circle by $f_{\lambda, d}^{N}$.

We now describe an algorithm that, on input $\hat{\lambda} \in \mathbb{S}_{\mathbb{Q}}$ and rational $\epsilon>0$, yields in $\operatorname{poly}(\operatorname{size}(\hat{\lambda}, \epsilon))$ a rooted tree $\hat{T}$ in $\mathcal{T}_{d+1}$ with $\mathcal{O}\left(\log \left(\epsilon^{-1}\right)\right)$ vertices whose field has distance at most $\epsilon$ from $\hat{\lambda}$; we will account for the degree of the root later. We assume for convenience that $\epsilon \ll \ell(I)$.

The first step of the algorithm is to find $i \in\{1, \ldots, m\}$ such that $\hat{\lambda} \in \operatorname{Arc}\left[f_{\lambda, d}^{N}\left(x_{i-1}\right), f_{\lambda, d}^{N}\left(x_{i}\right)\right]$. We know that such an arc must exist because $I$ is mapped surjectively onto $\mathbb{S}$ by $f_{\lambda, d}^{N}$ and, since $f_{\lambda, d}^{N}(z)$ is a rational function of $z$ with fixed degree, we can find $i$ in time poly $(\operatorname{size}(\hat{\lambda}))$. Now we consider the bijective map

$$
f_{\lambda, d}^{N}: \operatorname{Arc}\left[x_{i-1}, x_{i}\right] \rightarrow \operatorname{Arc}\left[f_{\lambda, d}^{N}\left(x_{i-1}\right), f_{\lambda, d}^{N}\left(x_{i}\right)\right]
$$

Analogously, with $n=\left\lceil\log _{3 / 2}\left(\ell\left(\operatorname{Arc}\left[x_{i-1}, x_{i}\right]\right) \cdot C_{2}^{N} / \epsilon\right)\right\rceil$ applications of $f_{\lambda, d}^{N}$, we can determine using binary search in time poly $(\operatorname{size}(\hat{\lambda}, \epsilon))$ an $\operatorname{arc} J \subseteq \operatorname{Arc}\left[x_{i-1}, x_{i}\right]$ with endpoints in $\mathbb{S}_{\mathbb{Q}}$ such that $\hat{\lambda} \in f_{\lambda, d}^{N}(J)$ and whose length satisfies

$$
3^{-n} \cdot \ell\left(\operatorname{Arc}\left[x_{i-1}, x_{i}\right]\right) \leq \ell(J) \leq(2 / 3)^{n} \cdot \ell\left(\operatorname{Arc}\left[x_{i-1}, x_{i}\right]\right) \leq \epsilon / C_{2}^{N} .
$$

Note that the length of $J$ is bounded below by $C_{3} \cdot \epsilon^{5}$, where $C_{3}$ is a constant independent of $\hat{\lambda}$ or $\epsilon$. It follows from (15) that $\ell\left(f_{\lambda, d}^{N}(J)\right) \leq \epsilon$, which means that the arc $J$ is mapped by $f_{\lambda, d}^{N}$ to an arc of length at most $\epsilon$ that includes $\hat{\lambda}$. We will next show how to construct in $\operatorname{poly}(\operatorname{size}(\hat{\lambda}, \epsilon))$ a rooted tree $T$ in $\mathcal{T}_{d+1}$ with $s=\mathcal{O}\left(\log \left(\epsilon^{-1}\right)\right)$ vertices that implements a field $w \in J$. Then, using Lemma $4,{ }^{8}$ we obtain a rooted tree $\hat{T}$ with $\left(d^{N}-1\right) /(d-1)+d^{N} s$ vertices that implements the field $\lambda^{\prime}=f_{\lambda, d}^{N}(w)$ with $\left|\lambda^{\prime}-\hat{\lambda}\right| \leq \epsilon$.

To construct $T$, we first fix some constants. Let $C_{4}=\left|f_{1}^{\prime}\left(R_{1}\left(\xi_{1}\right)\right)\right|$ and $C_{5}=\left|f_{1}^{\prime}\left(R_{1}\left(\xi_{2}\right)\right)\right|$ and note that $C_{4}, C_{5} \in\left(\frac{1}{2}, 1\right)$. We also have $C_{4} \leq\left|f_{1}^{\prime}(z)\right| \leq C_{5}$ for all $z \in I$. It follows that $f_{\xi_{2}, 1}(I)=$ $\operatorname{Arc}\left[f_{\xi_{2}, 1}\left(R_{1}\left(\xi_{1}\right)\right), R_{1}\left(\xi_{2}\right)\right]$ is contained in $I$ and its length is strictly bigger than $\ell(I) / 2$. Furthermore, it follows that $f_{\xi_{1}, 1}^{-1}\left(\operatorname{Arc}\left[R_{1}\left(\xi_{1}\right), f_{\xi_{2}, 1}\left(R_{1}\left(\xi_{1}\right)\right)\right]\right)=\operatorname{Arc}\left[R_{1}\left(\xi_{1}\right), f_{\xi_{1}}^{-1}\left(f_{\xi_{2}}\left(R_{1}\left(\xi_{1}\right)\right)\right)\right]$ is strictly contained inside $I$. Let $J_{0}=J$, and for $k \geq 0$, as long as $f_{\xi_{2}, 1}\left(R_{1}\left(\xi_{1}\right)\right) \notin J_{k}$, define

$$
J_{k+1}= \begin{cases}f_{\xi_{1}, 1}^{-1}\left(J_{k}\right) & \text { if } J_{k} \subset \operatorname{Arc}\left[R_{1}\left(\xi_{1}\right), f_{\xi_{2}, 1}\left(R_{1}\left(\xi_{1}\right)\right)\right] \\ f_{\xi_{2}, 1}^{-1}\left(J_{k}\right) & \text { if } \left.J_{k} \subset \operatorname{Arc}\left[f_{\xi_{2}, 1}\left(R_{1}\left(\xi_{1}\right)\right)\right), R_{1}\left(\xi_{2}\right)\right] .\end{cases}
$$

We have that $J_{k} \subseteq I$ for every $k$ and $\ell\left(J_{k}\right) \geq C_{5}^{-k} \cdot \ell\left(J_{0}\right) \geq C_{3} \cdot C_{5}^{-k} \cdot \epsilon^{5}$. Because $C_{5}<1$, we deduce that there is $N_{1} \geq 0$ such that $f_{\xi_{2}, 1}\left(R_{1}\left(\xi_{1}\right)\right) \in J_{N_{1}}$ where $N_{1}$ is bounded above by

$$
\left\lceil\frac{\log \left(C_{3} \cdot \epsilon^{5} / \ell(I)\right)}{\log \left(C_{5}\right)}\right\rceil=\mathcal{O}\left(\log \left(\epsilon^{-1}\right)\right) .
$$

\footnotetext{
${ }^{8}$ Lemma 4 describes how to construct a tree of size $s \cdot d+1$ with field $f_{\lambda, d}(z)$ from a tree of size $s$ and field $z$. Repeating this construction $N$ times yields the construction of $\hat{T}$ from $T$.
} 
Let $i_{1}, \ldots, i_{N_{1}}$ be the sequence of indices such that $f_{\xi_{i_{k}}}\left(J_{k}\right)=J_{k-1}$ and note that these can be computed in $\operatorname{poly}(\operatorname{size}(\hat{\lambda}, \epsilon))$ time. Let $K=f_{\xi_{2}, 1}^{-1}\left(J_{N_{1}}\right)$. We see that $R_{1}\left(\xi_{1}\right) \in K$ and

$$
\left(f_{\xi_{i_{1}}, 1} \circ \cdots \circ f_{\xi_{i_{1}}, 1} \circ f_{\xi_{2}, 1}\right)(K)=J .
$$

Furthermore, because the maps $f_{\xi_{i}, 1}^{-1}$ are expanding on $I$, we find $\ell(K) \geq \ell(J) \geq C_{3} \cdot \epsilon^{5}$. This means that there is an arc of length at least $\frac{1}{2} \cdot C_{3} \cdot \epsilon^{5}$ extending from $R_{1}\left(\xi_{1}\right)$, going either clockwise or counterclockwise, contained in $K$. In the case that such a clockwise arc exists - that is, $\operatorname{Arc}\left[R_{1}\left(\xi_{1}\right)\right.$. $\left.e^{-i \frac{1}{2} C_{3} \epsilon^{5}}, R_{1}\left(\xi_{1}\right)\right] \subseteq K$ - we see that, because $R_{1}$ is an attracting fixed point of $f_{\xi_{1}}$, there is some $N_{2}$, specified below, such that $f_{\xi_{1}, 1}^{N_{2}}\left(\xi_{1}\right) \in K$. Using that for integers $n$ we have

$$
\ell\left(\operatorname{Arc}\left[f_{\xi_{1}, 1}^{n}\left(\xi_{1}\right), R_{1}\left(\xi_{1}\right)\right]\right)=\ell\left(f_{\xi_{1}, 1}^{n}\left(\operatorname{Arc}\left[\xi_{1}, R_{1}\left(\xi_{1}\right)\right]\right)\right) \leq C_{4}^{n} \cdot \ell\left(\operatorname{Arc}\left[\xi_{1}, R_{1}\left(\xi_{1}\right)\right]\right)<C_{4}^{n} \cdot 2 \pi,
$$

we see that it suffices to take $N_{2}=\left\lceil\frac{\log \left(C_{3} \cdot \epsilon^{5} /(4 \pi)\right)}{\log \left(C_{4}\right)}\right\rceil=\mathcal{O}\left(\log \left(\epsilon^{-1}\right)\right)$. In the case that such a clockwise arc does not exist, we find that a counterclockwise arc of length $\frac{1}{2} \cdot C_{3} \cdot \epsilon^{5}$ is contained in $K$. Note that there is some integer $N_{c}$ independent of $\hat{\lambda}$ and $\epsilon$ such that $f_{\xi_{2}, 1}^{N_{c}}\left(\xi_{1}\right) \in I$. The same analysis as above shows that then $\left(f_{\xi_{1}, 1}^{N_{2}} \circ f_{\xi_{2}, 1}^{N_{c}}\right)\left(\xi_{1}\right) \in K$. We let $N_{3}$ be equal to zero if a clockwise arc of sufficient length is contained in $K$ and, otherwise, we let $N_{3}=N_{c}$. We conclude that

$$
\left(f_{\lambda, d}^{N} \circ f_{\xi_{i_{1}}, 1} \circ \cdots \circ f_{\xi_{N_{N_{1}}}, 1} \circ f_{\xi_{2}, 1} \circ f_{\xi_{1}, 1}^{N_{2}} \circ f_{\xi_{2}, 1}^{N_{3}}\right)\left(\xi_{1}\right)
$$

has a distance at most $\epsilon$ from $\hat{\lambda}$. By repeatedly applying the constructions laid out in Lemma 4 (cf. Footnote 8), we conclude that we can construct a tree $T$ in $\mathcal{T}_{d+1}$ whose field is given by the value in (16) and with $\mathcal{O}\left(\log \left(\epsilon^{-1}\right)\right)$ vertices.

This finishes the description of the algorithm, modulo that the root of the tree we constructed has degree $d$. To obtain a rooted tree with root degree 1 , we run the algorithm described on input $f_{\lambda, 1}^{-1}(\hat{\lambda})$ and $\epsilon \cdot \frac{d-1}{d+1}$ to obtain a rooted tree with root degree $d$ and field $\zeta$ with $\left|f_{\lambda, 1}^{-1}(\hat{\lambda})-\zeta\right|<\epsilon \cdot \frac{d-1}{d+1}$. Attaching one new vertex by an edge to this root yields a rooted tree with root degree 1 and field $f_{\lambda, 1}(\zeta)$ which satisfies, using Item i of Lemma 10, that

$$
\left|\hat{\lambda}-f_{\lambda, 1}(\zeta)\right| \leq\left|f_{\lambda, 1}^{-1}(\hat{\lambda})-\zeta\right| \cdot \max _{z \in S}\left|f_{\lambda, 1}^{\prime}(z)\right|<\epsilon \cdot \frac{d-1}{d+1} \cdot \frac{d+1}{d-1}=\epsilon,
$$

as wanted. This finishes the proof of Lemma 32.

\section{Reduction}

In this section, we prove our inapproximability results. Throughout this section, we use $\mathcal{G}_{\Delta}$ to denote the set of all graphs with maximum degree at most $\Delta$. We start in Subsection 8.1 with some preliminaries that will be used in our proofs. Subsection 8.2 gives the main reduction, and we show how to use this in Subsection 8.3 to conclude the proof of Theorem 1.

\subsection{Preliminaries}

We will use the following lemma from [42].

Lemma 33 ([42]). Let $\Delta \geq 3$ be an integer and let $\lambda \in \mathbb{S}_{\mathbb{Q}}$ with $\lambda \neq-1$. Then, there exists $\eta=\eta(\Delta, \lambda)>1$ such that, for all $b \in(1 / \eta, \eta)$, for all graphs $G \in \mathcal{G}_{\Delta}$, it holds that $Z_{G}(\lambda, b) \neq 0$.

For a graph $G$ and vertices $u, v$ in $G$, let $Z_{G, \pm u, \pm v}(\lambda, b)$ denote the contribution to the partition function when $u, v$ are assigned the spins \pm , respectively. For a configuration $\sigma$ on $G$, we use $w_{G, \sigma}(\lambda, b)$ to denote the weight $\lambda^{\left|n_{+}(\sigma)\right|} b^{\delta(\sigma)}$ of $\sigma$. We will use the following observation. 
Lemma 34. Let $\lambda \in \mathbb{S}$ and $b \in \mathbb{R}$. Then, for an arbitrary graph $G=\left(V_{G}, E_{G}\right)$ and vertices $u, v$ of $G$, it holds that

$$
Z_{G,+u,+v}(\lambda, b)=\lambda^{|V(G)|} \overline{Z_{G,-u,-v}(\lambda, b)}, \quad Z_{G,+u,-v}(\lambda, b)=\lambda^{|V(G)|} \overline{Z_{G,-u,+v}(\lambda, b)} .
$$

Proof. For an assignment $\sigma: V_{G} \rightarrow\{+,-\}$, let $\bar{\sigma}: V_{G} \rightarrow\{+,-\}$ be the assignment obtained by interchanging the assignment of +'s with -'s. Then

$$
w_{G, \bar{\sigma}}(\lambda, b)=\lambda^{\left|n_{+}(\bar{\sigma})\right|} b^{\delta(\bar{\sigma})}=\lambda^{\left|V_{G}\right|-\left|n_{+}(\sigma)\right|} b^{\delta(\sigma)}=\lambda^{|V(G)|} \overline{w_{G, \sigma}(\lambda, b)} .
$$

The result follows by summing over the relevant $\sigma$ for each of $Z_{G,+u,+v}(\lambda, b)$ and $Z_{G,+u,-v}(\lambda, b)$.

The following lemma will be useful in general for handling rational points on the circle. Ideally, we would like to describe a number on $\mathbb{S}$ by a rational angle, but this may not correspond to a rational Cartesian point, which would complicate computations. However, rational points are dense on the circle, and we can compute one arbitrarily close to a given angle, as follows.

Lemma 35. Given a rational angle $\theta \in[0,2 \pi)$ and $\epsilon \in(0,1)$, there exists a number $\hat{\theta}$ such that $|\theta-\hat{\theta}|<\epsilon$ and $\cos \hat{\theta}, \sin \hat{\theta} \in \mathbb{Q}$ are rational numbers of size at most poly $(\operatorname{size}(\theta, \epsilon))$. Furthermore, we can compute $\cos \hat{\theta}$ and $\sin \hat{\theta}$ in time poly $(\operatorname{size}(\theta, \epsilon))$.

Proof. By symmetry, we may assume that $\theta \in[0, \pi / 4]$. Given $\theta$, take a rational approximation $r$ of $\tan (\theta / 2)$ such that $|\tan (\theta / 2)-r|<\epsilon / 2$. We claim that $\hat{\theta}=2 \arctan (r)$ has the desired properties.

Write $s, c, t$ respectively for $\sin \hat{\theta}, \cos \hat{\theta}, \tan \hat{\theta}$. Using the tan double angle formula, we have $s / c=t=$ $2 r /\left(1-r^{2}\right)$. We also know that $s^{2}+c^{2}=1$. Solving these simultaneously gives that $s=2 r /\left(1+r^{2}\right)$ and $c=\left(1-r^{2}\right) /\left(1+r^{2}\right)$, which are both rational since $r$ is rational.

Also, writing $f(x)=2 \arctan (x)$ for $x \in[0,1]$, note that $f^{\prime}(x)=2 /\left(1+x^{2}\right) \in[1,2]$ for $x \in[0,1]$. Hence, $|f(x)-f(y)| \leq 2|x-y|$ for $x, y \in[0,1]$ and so $\left|\theta-\theta^{\prime}\right|<\epsilon$.

Finally, we can compute $r$ in poly $(\operatorname{size}(\theta, \epsilon))$ using a series expansion of tan from which we can compute $s$ and $c$ from the formulas above.

Finally, we will use the following well-known lemma for continued-fraction approximation.

Lemma 36 ([43, Corollary 6.3a]). There is a poly-time algorithm which, on input a rational number $\alpha$ and integer $K \geq 1$, decides whether there exists a rational number $p / q$ with $1 \leq q \leq K$ and $|\alpha-(p / q)|<1 / 2 K^{2}$ and, if so, finds this (unique) rational number.

\subsection{The reduction}

To prove Theorem 1, we will show how to use a poly-time algorithm for \#lsingNorm $(\lambda, b, \Delta, K)$ and \#Ising $\operatorname{Arg}(\lambda, b, \Delta, \rho)$ to compute exactly $Z_{G}(\lambda, \hat{b})$ on graphs $G$ of maximum degree 3 for some appropriate value of $\hat{b}$ that we next specify.

Let $\eta=\eta(3, \lambda)>1$ be as in Lemma 33, so that

$$
Z_{G}\left(\lambda, b^{\prime}\right) \neq 0 \text { for all } b^{\prime} \in(1 / \eta, \eta) \text { and } G \in \mathcal{G}_{3} \text {. }
$$

For $k=2,3, \ldots$, let $P_{k}$ be the path with $k$ vertices whose endpoints are labelled $u_{k}, v_{k}$ and all vertex activities are equal to 1 . Then, it is not hard to see that

$$
\left[\begin{array}{ll}
Z_{P_{k},+u_{k},+v_{k}}(1, b) & Z_{P_{k},+u_{k},-v_{k}}(1, b) \\
Z_{P_{k},-u_{k},+v_{k}}(1, b) & Z_{P_{k},-u_{k},-v_{k}}(1, b)
\end{array}\right]=\left[\begin{array}{ll}
1 & b \\
b & 1
\end{array}\right]^{k-1} .
$$


Clearly, for all $k$ it holds that

$$
Z_{P_{k},+u_{k},+v_{k}}(1, b)=Z_{P_{k},-u_{k},-v_{k}}(1, b) \text { and } \frac{Z_{P_{k},+u_{k},-v_{k}}(1, b)}{Z_{P_{k},-u_{k},-v_{k}}(1, b)}=\frac{Z_{P_{k},-u_{k},+v_{k}}(1, b)}{Z_{P_{k},-u_{k},-v_{k}}(1, b)}=: b_{k} .
$$

Moreover, using (18), we have that there exists $k$ such that

$$
1 / \eta<\hat{b}=b_{k}<\eta
$$

By the choice of $k$ and (17), we conclude that

$$
Z_{G}(\lambda, \hat{b}) \neq 0 \text { for all } G \in \mathcal{G}_{3} \text {. }
$$

The main step in the reduction is captured by the following lemma.

Lemma 37. Let $\Delta \geq 3$ be an integer, $b \in(0,1)$ be a rational and let $\lambda \in \mathbb{C}_{\mathbb{Q}}(\Delta-1, b)$ Let $K=$ 1.001 and $\rho=\pi / 40$. Assume that a poly-time algorithm exists for either \#Ising $\operatorname{Norm}(\lambda, b, \Delta, K)$ or \#Ising $\operatorname{Arg}(\lambda, b, \Delta, \rho)$. Then, there exists a poly-time algorithm that on input a graph $G \in \mathcal{G}_{3}$ and an edge $e=\{u, v\}$ of $G$ outputs the value of the ratio

$$
R_{G, e}=\frac{\hat{b}^{2} z_{++}+\hat{b}\left(z_{+-}+z_{-+}\right)+z_{--}}{\hat{b}^{2} z_{--}+\hat{b}\left(z_{+-}+z_{-+}\right)+z_{++}}, \text {where } z_{ \pm \pm}:=Z_{G \backslash e, \pm u, \pm v}(\lambda, \hat{b}) .
$$

The algorithm also outputs the value of the ratio $R_{G, e}^{\prime}=z_{--} / z_{++}$, provided that $z_{++} \neq 0$.

Remark 38. As will be shown in the proof of Lemma 37, the ratio $R_{G, e}$ is well-defined for all graphs $G \in \mathcal{G}_{3}$ and edges $e$ in $G$ using the zero-free region in Lemma 33 and the choice of $\hat{b}$. It is harder to show that $R_{G, e}^{\prime}$ is well-defined (we cannot use Lemma 33 directly) and hence the need for the assumption that $z_{++} \neq 0$ in Lemma 37.

Proof. Suppose that $G=(V, E)$ with $n=|V|$ and $m=|E|$. Let

$$
\begin{aligned}
r=\hat{b}^{2} z_{++}+\hat{b}\left(z_{+-}+z_{-+}\right)+z_{--}, & r^{\prime}=\left(\hat{b}^{2}-1\right)^{2} z_{--} \\
t=\hat{b}^{2} z_{--}+\hat{b}\left(z_{+-}+z_{-+}\right)+z_{++}, & t^{\prime}=\left(\hat{b}^{2}-1\right)^{2} z_{++}
\end{aligned}
$$

We first show that $r, t \neq 0$. Consider the graph $H=\left(V_{H}, E_{H}\right)$ obtained from $G$ by subdividing edge $e$; that is, we remove edge $e=\{u, v\}$ and then add a new vertex $s$ which is connected to both $u, v$. Note that $H$ is obtained from $G \backslash e$ by adding the edges $\{s, u\},\{s, v\}$, so it is not hard to see that

$$
Z_{H}(\lambda, \hat{b})=\lambda t+r
$$

Note that $H$ is a graph of maximum degree $\Delta$ and we have $Z_{H}(\lambda, \hat{b}) \neq 0$ from (21). Moreover, from Lemma 34, we have $r=\lambda^{n} \bar{t}$. Combining these, we obtain that $r, t \neq 0$. From assumption, we also have that $t^{\prime} \neq 0$.

We will show how to compute the ratios $R_{\text {goal }}=-\frac{r}{t}$ and $R_{\text {goal }}^{\prime}=-\frac{r^{\prime}}{t^{\prime}}$ (note that these are well-defined since $t, t^{\prime} \neq 0$ ). By Lemma 34, we have that $r=\lambda^{n} \bar{t}$ and $r^{\prime}=\lambda^{n} \overline{t^{\prime}}$, so $R_{\text {goal }}, R_{\text {goal }}^{\prime} \in \mathbb{S}_{\mathbb{Q}}$. In fact, letting $p, p^{\prime}, p^{\prime \prime}, q$ be integers such that $\hat{b}=p / q$ and $\lambda=\left(p^{\prime}+\mathrm{i} p^{\prime \prime}\right) / q$, we have that $R_{\text {goal }}, R_{\text {goal }}^{\prime} \in \mathcal{R} \cap \mathbb{S}_{\mathbb{Q}}$, where

$$
\mathcal{R}=\left\{\frac{P+\mathrm{i} Q}{P^{\prime}+\mathrm{i} Q^{\prime}} \mid P, Q, P^{\prime}, Q^{\prime} \in\{-M, \ldots, 0, \ldots, M\}\right\} \text { and } M:=2^{n}|p|^{m}\left(\left|p^{\prime}\right|+\left|p^{\prime \prime}\right|\right)^{n} q^{m+n} .
$$


Let $\epsilon=1 /(10 M)^{16}$. Note that for any two distinct numbers $z, z^{\prime} \in \mathcal{R}$ it holds that $\left|z-z^{\prime}\right| \geq 10 \epsilon$, so if we manage to produce $\hat{R}, \hat{R}^{\prime} \in \mathbb{S}_{\mathbb{Q}}$ with $\operatorname{poly}(n)$ size so that $\left|R_{\text {goal }}-\hat{R}\right| \leq \epsilon$ and $\left|R_{\text {goal }}^{\prime}-\hat{R}^{\prime}\right| \leq \epsilon$, we can in fact compute $R_{\text {goal }}$ and $R_{\text {goal }}^{\prime}$ in time $\operatorname{poly}(n, \operatorname{size}(\epsilon))=\operatorname{poly}(n) .{ }^{9}$

We first focus on how to compute $\hat{R} \in \mathbb{S}_{\mathbb{Q}}$ so that $\left|R_{\text {goal }}-\hat{R}\right| \leq \epsilon$. At this point, it will be helpful to represent complex numbers on the unit circle $\mathbb{S}$ with their arguments. Let $\theta_{\text {goal }}=\operatorname{Arg}\left(R_{\text {goal }}\right)$ and $g(\theta):=t \mathrm{e}^{\mathrm{i} \theta}+r$. Note that

$$
\begin{aligned}
& |g(\theta)|=\left|g(\theta)-g\left(\theta_{\text {goal }}\right)\right|=|t|\left|\mathrm{e}^{\mathrm{i} \theta}-\mathrm{e}^{\mathrm{i} \theta_{\text {goal }}}\right|=2|t|\left|\sin \left(\left(\theta-\theta_{\text {goal }}\right) / 2\right)\right|, \\
& \operatorname{Arg}(g(\theta))=\left(\theta-\theta_{\text {goal }}\right) / 2+\operatorname{Arg}(t) \bmod 2 \pi,
\end{aligned}
$$

the latter provided $\theta \neq \theta_{\text {goal }}$.

We will compute in $\operatorname{poly}(n)$ time a rational $\hat{\theta}$ such that $\left|\hat{\theta}-\theta_{\text {goal }}\right| \leq \epsilon / 2$, yielding the desired $\hat{R}$ (via Lemma 35).

Let $\tau=1 / 500$ and $\kappa=\epsilon / 10^{3}$. We will show that a poly-time algorithm for \#IsingNorm $(\lambda, b, \Delta, K)$ can be used to compute, for every rational $\theta$, a positive number $\hat{g}_{\theta}$ in time $\operatorname{pol} y(n, \operatorname{size}(\theta))$ such that, whenever $|\theta-a| \geq \kappa$ for every $a \in \arg \left(R_{\text {goal }}\right)$, it holds that

$$
(1-\tau)|g(\theta)| \leq \hat{g}_{\theta} \leq(1+\tau)|g(\theta)| .
$$

When $|\theta-a| \leq \kappa$ for some $a \in \arg \left(R_{\text {goal }}\right)$, there is no guarantee on the value of $\hat{g}_{\theta}$. Similarly, we will show that a poly-time algorithm for \#lsing $\operatorname{Arg}(\lambda, b, \Delta, \rho)$ can be used to compute, for every rational $\theta$, a positive number $\hat{a}_{\theta}$ in time $\operatorname{poly}(n, \operatorname{size}(\theta))$ such that, whenever $|\theta-a| \geq \kappa$ for every $a \in \arg \left(R_{\text {goal }}\right)$, it holds that

$$
\left|\operatorname{Arg}(g(\theta))-\hat{a}_{\theta}\right| \leq 2 \rho=\pi / 20 .
$$

Using these, we compute the desired $\hat{\theta}$ via binary search following techniques similar to those in $[18,20,6]$, though in our case the details are a bit different because we have to work on the unit circle. For the norm, we will utilise that $|g(\theta)|$ is increasing in the interval $\left[\theta_{\text {goal }}, \theta_{\text {goal }}+\pi\right]$ and decreasing in the interval $\left[\theta_{\text {goal }}-\pi, \theta_{\text {goal }}\right]$, whereas for the argument we will utilise that $\operatorname{Arg}(g(\theta))$ changes abruptly around $\theta_{\text {goal }}$ (roughly by $\pi$ ). In particular, we proceed as follows.

Algorithm for \#IsingNorm $(\lambda, b, \Delta, K)$ (Step 1): We first find an interval of length $<2 \pi / 3$ with rational endpoints containing $\theta_{\text {goal }}$ in $\operatorname{poly}(n)$ time. For $j=0, \ldots, 18$, let $\theta_{j}=j / 3, g_{j}=\left|g\left(\theta_{j}\right)\right|$ and $\hat{g}_{j}=\hat{g}_{\theta_{j}}$; note that the $\hat{g}_{j}$ s can be computed in $\operatorname{poly}(n)$ time. For convenience, extend these definitions by setting $\theta_{19 h+j}=\theta_{j}+2 h \pi, g_{19 h+j}=g_{j}$ and $\hat{g}_{19 h+j}=\hat{g}_{j}$ for every integer $h$ and $j=0, \ldots, 18$. Note that for all $j$ we have that $1 / 3 \geq\left|\theta_{j+1}-\theta_{j}\right| \geq 1 / 4>\pi / 15$.

Consider an index $j \in\{0, \ldots, 18\}$ such that $\arg \left(R_{\text {goal }}\right)$ does not intersect with the intervals $\left[\theta_{j}-\right.$ $\left.\kappa, \theta_{j+1}+\kappa\right]$ and $\left[\theta_{j}-\pi, \theta_{j+1}-\pi\right]$. Then, we have that

$$
(1-\tau) g_{j} \leq \hat{g}_{j} \leq(1+\tau) g_{j}, \quad(1-\tau) g_{j+1} \leq \hat{g}_{j+1} \leq(1+\tau) g_{j+1} .
$$

We claim that $g_{j+1}-g_{j}$ has the same sign as $\hat{g}_{j+1}-\hat{g}_{j}$. To see this, assume w.l.o.g. $g_{j+1}-g_{j}>0$ that the other possibility follows in a similar way. Observe that we must have $\theta_{j}, \theta_{j+1} \in\left(\theta_{\text {goal }}, \theta_{\text {goal }}+\pi\right)$, as

\footnotetext{
${ }^{9}$ We briefly give the details for $R_{\text {goal }}$; the details for $R_{\text {goal }}^{\prime}$ are similar. For $r \in \mathbb{N}$ let $\mathbb{Q}_{r}$, denote the set of rationals with denominator between 1 and $r$. Since $R_{\text {goal }} \in \mathcal{R} \cap \mathbb{S}_{\mathbb{Q}}$ and $\hat{R} \in \mathbb{S}_{\mathbb{Q}}$, we have that there exist $\alpha, \beta \in \mathbb{Q}_{2 M^{2}}$ and $\hat{\alpha}, \hat{\beta} \in \mathbb{Q}$ such that $R_{\text {goal }}=\alpha+\mathrm{i} \beta$ and $\hat{R}=\hat{\alpha}+\mathrm{i} \hat{\beta}$. From $\left|R_{\text {goal }}-\hat{R}\right| \leq \epsilon$, we have $|\alpha-\hat{\alpha}|,|\beta-\hat{\beta}| \leq \epsilon$. By Lemma 36 (applied to $\hat{\alpha}, \hat{\beta}$ and $\left.K=2 M^{2}\right)$, in poly(n) time, we can compute rationals $\alpha^{\prime}, \beta^{\prime} \in \mathbb{Q}_{2 M^{2}}$ such that $\left|\hat{\alpha}-\alpha^{\prime}\right|,\left|\hat{\beta}-\beta^{\prime}\right| \leq 1 /\left(8 M^{4}\right)$ and hence $\left|\alpha-\alpha^{\prime}\right|,\left|\beta-\beta^{\prime}\right| \leq \epsilon+1 /\left(8 M^{4}\right) \leq 1 /\left(4 M^{4}\right)$. Now, for distinct $\gamma, \delta \in \mathbb{Q}_{2 M^{2}}$, we have that $|\gamma-\delta| \geq 1 /\left(2 M^{2}\right)$, so it must be that $\alpha=\alpha^{\prime}$ and $\beta=\beta^{\prime}$, completing the computation of $R_{\text {goal }}$.
} 
$\theta \mapsto \sin \left(\theta / 2-\theta_{\text {goal }} / 2\right)$ is increasing on $\left(\theta_{\text {goal }}, \theta_{\text {goal }}+\pi\right)$, and so

$$
\begin{aligned}
g_{j+1}-g_{j} & =\left|g\left(\theta_{j+1}\right)\right|-\left|g\left(\theta_{j}\right)\right| \geq 2|t| \min _{\phi \in[0, \pi / 2-\pi / 30)]}[\sin (\phi+\pi / 30)-\sin \phi] \\
& \geq 2|t|[\sin (\pi / 2)-\sin (\pi / 2-\pi / 30)] \geq|t| / 100 .
\end{aligned}
$$

On the other hand, if $\hat{g}_{j+1}-\hat{g}_{j}<0$, from (26) we have $(1-\tau) g_{j+1}-(1+\tau) g_{j}<0$. This gives $g_{j+1}-g_{j} \leq \tau\left(g_{j+1}+g_{j}\right) \leq 2 \tau|t|$, a contradiction to the above.

Let $j^{*}$ be such that $\theta_{\text {goal }} \in\left[\theta_{j^{*}}, \theta_{j^{*}+1}\right)$. From (23), the sequence $g_{j}$ is decreasing until $j^{*}$ and increasing after $j^{*}+1$. From the claim above, the sequence $\hat{g}_{j}$ must therefore be decreasing for indices $j$ in $\left[j^{*}-8, j^{*}-1\right]$ and increasing for indices $\left[j^{*}+2, j^{*}+9\right]$. Therefore, from the values of $\hat{g}_{j}$ s we can find $\hat{j}$ so that $\theta_{\text {goal }} \in\left[\theta_{\hat{j}-3}, \theta_{\hat{j}+3}\right]$. By enlarging slightly the interval $\left[\theta_{\hat{j}-3}, \theta_{\hat{j}+3}\right]$, we obtain the desired interval of length $<2 \pi / 3$ with rational endpoints.

Algorithm for \#IsingNorm $(\lambda, b, \Delta, K)$ (Step 2): Given an interval $\left[\theta_{1}, \theta_{2}\right]$ with rational endpoints containing $\theta_{\text {goal }}$ with $\left|\theta_{1}-\theta_{2}\right|=\ell$ and $\ell \in(100 \kappa, 2 \pi / 3)$, we show how to find in $\operatorname{poly}\left(n, \operatorname{size}\left(\theta_{1}, \theta_{2}\right)\right)$ time an interval with rational endpoints that is a factor of $1 / 2$ smaller in length and also contains $\theta_{\text {goal }}$. The analysis will be similar to step 1.

For $j=0, \ldots, 19$ define $\phi_{j}=\theta_{1}+\left(\theta_{2}-\theta_{1}\right) j / 19$ and let $g_{j}=\left|g\left(\phi_{j}\right)\right|$ and $\hat{g}_{j}=\hat{g}_{\phi_{j}}$. Since $\theta_{\text {goal }} \in\left[\theta_{1}, \theta_{2}\right]$ and $\left|\theta_{1}-\theta_{2}\right|=\ell$, for any $\theta \in\left[\theta_{1}, \theta_{2}\right]$ we have $|g(\theta)| \leq 2|t| \sin (\ell / 2) \leq \ell|t|$. In particular, we have $g_{j} \leq \ell|t|$ for all $j$.

Moreover, for an index $j$ such that $\theta_{\text {goal }} \notin\left[\phi_{j}, \phi_{j+1}\right]$, we claim that $g_{j+1}-g_{j}$ has the same sign as $\hat{g}_{j+1}-\hat{g}_{j}$. To prove the claim, assume $g_{j+1}-g_{j} \geq 0$, so $\phi_{j+1}, \phi_{j} \geq \theta_{\text {goal }}$; the other possibility follows in a similar way. The derivative of $|g(\theta)|$ in the interval $\left[\theta_{\text {goal }}, \theta_{\text {goal }}+\ell\right]$ is bounded below by $|t| \cos (\ell / 2) \geq|t| / 2$, so by the mean value theorem we have that

$$
g_{j+1}-g_{j} \geq \frac{|t|}{2}\left(\phi_{j+1}-\phi_{j}\right) \geq|t| \ell / 50
$$

On the other hand, if $\hat{g}_{j+1}-\hat{g}_{j}<0$, then, as before, we have $(1-\tau) g_{j+1}-(1+\tau) g_{j}<0$, which implies $g_{j+1}-g_{j} \leq \tau\left(g_{j+1}+g_{j}\right) \leq 2 \tau \ell|t|$, a contradiction to the above. This proves the claim.

Using the claim, we can conclude just as we did in step 1 and find an index $\hat{j}$ so that $\theta_{\text {goal }} \in$ $\left[\phi_{\hat{j}-3}, \phi_{\hat{j}+3}\right]$, giving the desired interval.

Algorithm for \#Ising $\operatorname{Arg}(\lambda, b, \Delta, \rho)$ : Given a rational endpoint $\theta_{1}$ and a rational length $\ell \in\left(100 \kappa, \frac{63}{10}\right]$ such that $\theta_{\text {goal }}$ lies in the interval $\left[\theta_{1}, \theta_{2}\right]$ for some $\theta_{2} \leq \theta_{1}+\ell$, we show how to find in $\operatorname{pol} y\left(n, \operatorname{size}\left(\theta_{1}, \ell\right)\right)$ time a rational endpoint $\theta_{1}^{\prime}$ and a rational length $\ell^{\prime}$ such that $\ell^{\prime} \leq \ell / 4$ and $\theta_{\text {goal }} \in\left[\theta_{1}^{\prime}, \theta_{2}^{\prime}\right]$ for some $\theta_{2}^{\prime} \leq \theta_{1}^{\prime}+\ell^{\prime}$.

For $j=0, \ldots, 25$, define $\phi_{j}=\theta_{1}+\ell j / 26$ and let $a_{j}=\operatorname{Arg}\left(g\left(\phi_{j}\right)\right), \hat{a}_{j}=\hat{a}_{\phi_{j}}$. For convenience, extend these definitions by setting $\phi_{26 h+j}=\phi_{j}, a_{26 h+j}=a_{j}$ and $\hat{a}_{26 h+j}=\hat{a}_{j}$ for every integer $h$ and $j=0, \ldots, 25$. For indices $j, j^{\prime}$, let

$$
D_{j, j^{\prime}}=\min \left\{\left|a_{j^{\prime}}-a_{j}\right|, 2 \pi-\left|a_{j^{\prime}}-a_{j}\right|\right\} \text { and } \widehat{D}_{j, j^{\prime}}=\min \left\{\left|\hat{a}_{j^{\prime}}-\hat{a}_{j}\right|, 2 \pi-\left|\hat{a}_{j^{\prime}}-\hat{a}_{j}\right|\right\}
$$

Consider an index $j$ such that $\theta_{\text {goal }} \notin\left[\phi_{j}-\kappa, \phi_{j+1}+\kappa\right]$. Then, we have that $D_{j, j+1}=\left|\phi_{j+1}-\phi_{j}\right| / 2 \leq \pi / 10$ and hence $\widehat{D}_{j, j+1} \leq \pi / 5$. On the other hand, for an index $j$ such that $\theta_{\text {goal }} \in\left[\phi_{j}, \phi_{j+1}\right]$, we have that $D_{j-1, j+1}=\pi-\left|\phi_{j+1}-\phi_{j-1}\right| / 2 \geq 4 \pi / 5$ and, similarly, $D_{j, j+2} \geq 4 \pi / 5$. Therefore, at least one of $\widehat{D}_{j-1, j+1} \geq 3 \pi / 5, \widehat{D}_{j, j+2} \geq 3 \pi / 5$ must hold. Therefore, using the $\hat{a}_{j} \mathrm{~s}$, we can find an index $\hat{j}$ so that $\theta_{\text {goal }} \in\left[\phi_{\hat{j}-2}, \phi_{\hat{j}+2}\right]$, giving the desired interval.

By repeating the above, we conclude that, using a poly-time algorithm for either the problem \#IsingNorm $(\lambda, b, \Delta, K)$ or \#Ising $\operatorname{Arg}(\lambda, b, \Delta, \rho)$, we can compute in poly $(n)$ time a rational $\hat{\theta}$ such that 
$|\hat{\theta}-\theta| \leq 400 \kappa \leq \epsilon / 2$, yielding the desired $\hat{R}$ (via Lemma 35). We thus focus on proving that for a rational $\theta$ we can obtain in time $\operatorname{poly}(n, \operatorname{size}(\theta))$ values $\hat{g}_{\theta}, \hat{a}_{\theta}$ satisfying (24) and (25), respectively.

Let $\epsilon_{2}=\kappa \epsilon / 10^{5}, \epsilon_{1}:=\epsilon_{2} /\left(2^{4 n}(2 \hat{b})^{2 m}\right), \epsilon_{0}=\epsilon_{1} /\left(k 4^{k}\right)$. By Lemmas 32 and 35, for a rational number $\phi$, we can construct in time $\operatorname{poly}(n, \operatorname{size}(\phi))$ a rooted tree $T_{\phi}$ in $\mathcal{T}_{\Delta}$ with root $x_{\phi}$ that has degree 1 and implements a field $\lambda_{\phi}$ such that $\left|\lambda_{\phi}-\mathrm{e}^{\mathrm{i} \phi}\right| \leq \epsilon_{0}$. For convenience, let

$$
Q_{\phi}^{ \pm}:=Z_{T_{\phi}, \pm x_{\phi}}(\lambda, b) \text { and note that }\left|\frac{Q_{\phi}^{+}}{Q_{\phi}^{-}}-\mathrm{e}^{\mathrm{i} \phi}\right| \leq \epsilon_{0}
$$

Let $T_{\theta}, T_{0}$ be the trees obtained for $\phi=\theta, 0$ and note that that $T_{\theta}, T_{0}$ implement the vertex activities $\mathrm{e}^{\mathrm{i} \theta}, 1$, respectively (with precision $\epsilon_{0}$ ).

Recall that $P_{k}$ is the path with $k$ vertices and endpoints $u_{k}, v_{k}$, and we denote by $V_{P_{k}}$ the set of its vertices. Let $P_{k, T_{0}}$ be the tree obtained from $P_{k}$ by attaching $k-2$ disjoint copies of the graph $T_{0}$ to the internal vertices of the path; that is, for $i=1, \ldots, k-2$, identify the root $x_{0}$ of the $i$ th copy of $T_{0}$ with the $i$ th internal vertex of the path. For convenience, let

$$
A_{ \pm \pm}:=Z_{P_{k, T_{0}}, \pm u_{k}, \pm v_{k}}(\lambda, b)
$$

Recall that $H=\left(V_{H}, E_{H}\right)$ denotes the graph obtained by subdividing edge $e$ of $G$. Let $H_{\theta} \in \mathcal{G}_{\Delta}$ be the graph obtained from $H$ by replacing every edge $\{x, y\}$ of $H$ by a distinct copy of $P_{k, T_{0}}$ (identifying $x$ with $u_{k}$ and $y$ with $v_{k}$ ) and attaching the tree $T_{\theta}$ on the vertex $s$ of $H$ (identifying $s$ with the root $x_{\theta}$ ). Effectively, the construction of $H_{\theta}$ is so that the Ising model on $H_{\theta}$ with edge activities equal to $b$ and vertex activities equal to $\lambda$ corresponds to an Ising model on $H$ with edge activities equal to $\hat{b}$ and vertex activities equal to $\lambda$ apart from that of vertex $s$ which is set to $\mathrm{e}^{\mathrm{i} \theta}$. In this latter model, the contribution to the partition function from configurations where $s$ is set to + is given by $t$ and the contribution to the partition function from configurations where $s$ is set to - is given by $r$, where $t, r$ are as in (22). Based on this, we will soon show that

$$
\left|\frac{Z_{H_{\theta}}(\lambda, b)}{Q_{\theta}^{-}\left(A_{++}\right)^{m+1}}-g(\theta)\right| \leq \epsilon_{2}
$$

From (30), we obtain the desired approximations $\hat{g}_{\theta}, \hat{a}_{\theta}$ that satisfy (24), (25), respectively, as follows. First, observe that $|g(\theta)| \geq|t| \kappa / 2 \geq 10 \epsilon_{2} / \tau$ since $|\theta-a| \geq \kappa$ for every $a \in \arg \left(R_{\text {goal }}\right)$. Second, $T_{\theta}$ and $P_{k, T_{0}}$ are trees of size $\operatorname{poly}(n, \operatorname{size}(\theta))$, so we can compute $Q_{\theta}^{-}$and $A_{++}$in time $\operatorname{poly}(n, \operatorname{size}(\theta))$. Using a poly-time algorithm for \#IsingNorm $(\lambda, b, \Delta, K)$, we can compute $\hat{Z}_{\theta}$ in time poly $(n$, size $(\theta))$ which is within a factor of $1 \pm \tau$ from $\left|Z_{H_{\theta}}(\lambda, b)\right|$, thus yielding $\hat{g}_{\theta}=\frac{\hat{Z}_{\theta}}{\left|Q_{\theta}^{-}\right|\left|A_{++}\right|^{m+1}}$ that satisfies (24). Similarly, using a poly-time algorithm for \#lsingArg $(\lambda, b, \Delta, \rho)$, we can compute $\hat{A}_{\theta}$ in time poly $(n$, $\operatorname{size}(\theta))$ which is within distance $\rho$ from $\operatorname{Arg}\left(Z_{H_{\theta}}(\lambda, b)\right)$. Noting that the argument $\alpha$ of $\frac{Z_{H_{\theta}}(\lambda, b)}{Q_{\theta}^{-}\left(A_{++}\right)^{m+1}}-g(\theta)$ satisfies $\sin (\alpha) \leq \epsilon_{2} / g(\theta)$, from which it follows that $\alpha \leq \rho$. Hence, $\hat{a}_{\theta}=\hat{A}_{\theta}-\operatorname{Arg}\left(Q_{\theta}^{-}\right)-(m+$ 1) $\operatorname{Arg}\left(A_{++}\right)(\bmod 2 \pi)$ satisfies $(25)$.

It remains to prove (30). We first claim that

$$
\left|\frac{A_{ \pm \pm}}{\left(Q_{0}^{-}\right)^{k-2}}-Z_{P_{k}, \pm u_{k}, \pm v_{k}}(1, b)\right| \leq \frac{\epsilon_{1}}{4} Z_{P_{k}, \pm u_{k}, \pm v_{k}}(1, b) .
$$

Indeed, for a fixed $\sigma: V_{P_{k}} \rightarrow\{+,-\}$, the aggregate contribution to $Z_{P_{k, T_{0}}}(1, b)$ from configurations on $P_{k, T_{0}}$ that agree with $\sigma$ on $V_{P_{k}}$ is $\left(Q_{0}^{+}\right)^{n_{+}(\sigma)}\left(Q_{0}^{-}\right)^{n_{-}(\sigma)} w_{P_{k}, \sigma}(1, b)$ where $n_{ \pm}(\sigma)$ is the number of 
internal vertices in $P_{k}$ that have spin \pm under $\sigma$, so (31) follows from aggregating over the relevant $\sigma$ and observing that ${ }^{10}\left|\frac{\left(Q_{0}^{+}\right)^{j}}{\left(Q_{0}^{-}\right)^{j}}-1\right| \leq k \epsilon_{0}$ for all $j=0, \ldots, k$. From (19) and (31), it follows that $A_{ \pm, \pm} \neq 0$ and

$$
\left|\frac{A_{-+}}{A_{++}}-\hat{b}\right| \leq \epsilon_{1}, \quad\left|\frac{A_{--}}{A_{++}}-1\right| \leq \epsilon_{1} .
$$

Now, for $\sigma: V_{H} \rightarrow\{+,-\}$ with $\sigma(s)=+$, let $W_{\sigma}^{+}$be the aggregate weight of configurations on $H_{\theta}$ that agree with $\sigma$ on $V(H)$. Define analogously $W_{\sigma}^{-}$. Then, we have that

$$
W_{\sigma}^{ \pm}=Q_{\theta}^{ \pm}\left(A_{++}\right)^{m_{++}(\sigma)}\left(A_{+-}\right)^{m_{+-}(\sigma)}\left(A_{--}\right)^{m_{--}(\sigma)},
$$

where $m_{++}(\sigma), m_{+-}(\sigma), m_{--}(\sigma)$ denote the number edges of $E_{H}$ whose endpoints are assigned ,,+++--- , respectively. Since the total number of edges in $E_{H}$ is $m+1$, we obtain

$$
\left|\frac{W_{\sigma}^{+}}{Q_{\theta}^{-}\left(A_{++}\right)^{m+1}}-\mathrm{e}^{\mathrm{i} \theta} w_{H, \sigma}(\lambda, \hat{b})\right| \leq \epsilon_{2} / 10^{n}, \quad\left|\frac{W_{\sigma}^{-}}{Q_{\theta}^{-}\left(A_{++}\right)^{m+1}}-w_{H, \sigma}(\lambda, \hat{b})\right| \leq \epsilon_{2} / 10^{n} .
$$

Observe also that the quantities $t, r$, as defined in (22), are such that

$$
t=\sum_{\sigma: V_{H} \rightarrow\{+,-\} ; \sigma(s)=+} w_{H, \sigma}(\lambda, \hat{b}) \text { and } r=\sum_{\sigma: V_{H} \rightarrow\{+,-\} ; \sigma(s)=-} w_{H, \sigma}(\lambda, \hat{b}),
$$

so summing (33) over all $\sigma$ gives (30). This finishes the proof of (30) and hence completes the computation of $R_{\text {goal }}$ in $\operatorname{poly}(n)$ time.

The computation of $R_{\text {goal }}^{\prime}$ is completely analogous, once we establish an analogue of (30). In particular, let $H^{\prime}$ be the graph obtained from $H$ by removing vertex $s$ and adding the vertices $u^{\prime}, v^{\prime}, s^{\prime}$ and the edges $\left\{u, u^{\prime}\right\},\left\{u^{\prime}, s^{\prime}\right\},\left\{s^{\prime}, v^{\prime}\right\},\left\{v^{\prime}, v\right\}$; note that $H^{\prime}$ is obtained from $G$ by replacing the edge $e$ by a path with three vertices. We construct $H_{\theta}^{\prime}$ from $H^{\prime}$ as above, with a minor twist: we replace every edge $\{x, y\}$ of $H^{\prime}$ with a distinct copy of $P_{k, T_{0}}$ (identifying $x$ with $u_{k}$ and $y$ with $v_{k}$ ), we attach the rooted tree $T_{\theta}$ on the vertex $s^{\prime}$ of $H^{\prime}$ (identifying $s^{\prime}$ with the root $x_{\theta}$ ) and we attach two distinct copies of the rooted tree $T_{\pi}$ on the vertices $u^{\prime}, v^{\prime}$ of $H^{\prime}$ (identifying $u^{\prime}, v^{\prime}$ with the corresponding roots $x_{\pi}$ in the two copies of $T_{\pi}$ ). Note the use of the tree $T_{\pi}$ in the construction of $H^{\prime}$ which, analogous ${ }^{11}$ to (28), implements the field $\mathrm{e}^{\mathrm{i} \pi}=-1$ (with precision $\epsilon_{0}$ ). Effectively, the construction of $H_{\theta}^{\prime}$ is so that the Ising model on $H_{\theta}^{\prime}$ with edge activities equal to $b$ and vertex activities equal to $\lambda$ corresponds to an Ising model on $H^{\prime}$ with edge activities equal to $\hat{b}$ and vertex activities equal to $\lambda$ apart from those of $u^{\prime}, s^{\prime}, v^{\prime}$ which are set to $-1, \mathrm{e}^{\mathrm{i} \theta},-1$, respectively. In this latter model, the contribution to the partition function from configurations where $s^{\prime}$ is set to + is given by $t^{\prime}=\left(\hat{b}^{2}-1\right)^{2} z_{++}$and the contribution to the partition function from configurations where $s^{\prime}$ is set to - is given by $r^{\prime}=\left(\hat{b}^{2}-1\right)^{2} z_{--}$. Based on this, we obtain, similar to the above, the following analogue of (30):

$$
\left|\frac{Z_{H_{\theta}^{\prime}}(\lambda, b)}{Q_{\theta}^{-}\left(Q_{\pi}^{-}\right)^{2}\left(A_{++}\right)^{m+2}}-\left(t^{\prime} \mathrm{e}^{\mathrm{i} \theta}+r^{\prime}\right)\right| \leq \epsilon_{2}
$$

Having (34) at hand, the computation of $R_{\text {goal }}^{\prime}$ can be carried out using exactly the same procedure as for $R_{\text {goal }}$. This finishes the proof of Lemma 37 .

${ }^{10}$ Here and in the follow-up estimates, we use that for complex numbers $c_{1}, \ldots, c_{i}$ and $d_{1}, \ldots, d_{i}$ it holds that $\mid \prod_{j=1}^{i} c_{j}-$ $\prod_{j=1}^{i} d_{j}\left|\leq \sum_{j=1}^{i}\right| c_{j}-d_{j}\left|\prod_{j^{\prime}=1}^{j-1}\right| c_{j}\left|\prod_{j^{\prime}=j+1}^{i}\right| d_{j} \mid$.

${ }^{11}$ Even though $\pi$ is irrational, it holds that $\mathrm{e}^{\mathrm{i} \pi}=-1$, and we can therefore construct $T_{\pi}$ satisfying (28) for $\phi=\pi$ using Lemma 32. 


\subsection{Proof of our main theorem}

We are now ready to finish the proof of Theorem 1, which we restate here for convenience.

Theorem 1. Let $\Delta \geq 3$ be an integer and let $K=1.001$ and $\rho=\pi / 40$.

(a) Let $b \in\left(0, \frac{\Delta-2}{\Delta}\right]$ be a rational and $\lambda \in \mathbb{S}_{\mathbb{Q}}$ such that $\lambda \neq \pm 1$. Then the problems $\#$ \#singNorm $(\lambda, b, \Delta, K)$ and \#IsingArg $(\lambda, b, \Delta, \rho)$ are \#P-hard.

(b) Let $b \in\left(\frac{\Delta-2}{\Delta}, 1\right)$ be a rational. Then the collection of complex numbers $\lambda \in \mathbb{S}_{\mathbb{Q}}$ for which \#Ising Norm $(\lambda, b, \Delta, K)$ and \#Ising $\operatorname{Arg}(\lambda, b, \Delta, \rho)$ are \#P-hard is dense in the arc $\mathbb{S} \backslash I\left(\theta_{b}\right)$.

Proof of Theorem 1. Let $b \in(0,1)$ be a rational number and let $\lambda \in \mathbb{S}_{\mathbb{Q}}(\Delta-1, b)$. By Theorem 6 , it suffices to show that \#IsingNorm $(\lambda, b, \Delta, K)$ and \#IsingArg $(\lambda, b, \Delta, \rho)$ are \#P-hard. To prove the \#P-hardness for these problems, we will show that, assuming a poly-time algorithm for either \#IsingNorm $(\lambda, b, \Delta, K)$ or \#lsingArg $(\lambda, b, \Delta, \rho)$, on input of a graph $G \in \mathcal{G}_{3}$ we can compute $Z_{G}(\lambda, \hat{b})$ in poly-time, which is \#P-hard by [28, Theorem 1.1]. In fact, it suffices to compute in poly-time, for an arbitrary edge $e$ of $G$, the ratio $\frac{Z_{G}(\lambda, \hat{b})}{Z_{G \backslash e}(\lambda, \hat{b})}$, since then we can compute $Z_{G}(\lambda, \hat{b})$ using a telescoping product over the edges of the graph $G$.

So fix an arbitrary edge $e=\{u, v\}$ of $G$ and let $z_{ \pm \pm}:=Z_{G, \pm u, \pm v}(\lambda, \hat{b})$. The ratio $r^{*}:=\frac{Z_{G}(\lambda, \hat{b})}{Z_{G \backslash e}(\lambda, \hat{b})}$ is well-defined since, by the choice of $\hat{b}$, we have $Z_{G \backslash e}(\lambda, \hat{b}) \neq 0$ (cf. (20) and (21)). Moreover, we can express $r^{*}$ using the $z_{ \pm \pm} \mathrm{s}$ as follows:

$$
r^{*}=\frac{z_{++}+z_{--}+\hat{b}\left(z_{+-}+z_{-+}\right)}{z_{++}+z_{--}+z_{+-}+z_{-+}}
$$

We will compute $r^{*}$ using Lemma 37. Namely, by Lemma 37, we can compute in poly-time the value of the ratio

$$
r=R_{G, e}=\frac{A^{2} z_{++}+A B\left(z_{+-}+z_{-+}\right)+B^{2} z_{--}}{A^{2} z_{--}+A C\left(z_{+-}+z_{-+}\right)+C^{2} z_{++}}, \text {where } \begin{aligned}
& A:=\hat{b} \\
& B:=1 \\
& C:=1
\end{aligned}
$$

Let $G^{\prime}$ be the graph obtained from $G \backslash e$ by adding two new vertices $u^{\prime}$, $v^{\prime}$ and adding the edges $\left\{u, u^{\prime}\right\},\left\{u^{\prime}, v^{\prime}\right\},\left\{v^{\prime}, v\right\}$. We next apply Lemma 37 to the graph $G^{\prime}$ with the edge $e^{\prime}=\left\{u^{\prime}, v^{\prime}\right\}$. We first express $Z_{G^{\prime} \backslash e^{\prime}, \pm u^{\prime}, \pm v^{\prime}}(\lambda, \hat{b})$ in terms of the $z_{ \pm \pm}$s. We have

$$
\begin{aligned}
& Z_{G^{\prime} \backslash e^{\prime},+u^{\prime},+v^{\prime}}(\lambda, \hat{b})=\lambda^{2}\left(z_{++}+\hat{b}\left(z_{+-}+z_{-+}\right)+\hat{b}^{2} z_{--}\right), \\
& Z_{G^{\prime} \backslash e^{\prime},+u^{\prime},-v^{\prime}}(\lambda, \hat{b})=\lambda\left(\hat{b} z_{++}+z_{+-}+\hat{b}^{2} z_{-+}+\hat{b} z_{--}\right), \\
& Z_{G^{\prime} \backslash e^{\prime},-u^{\prime},+v^{\prime}}(\lambda, \hat{b})=\lambda\left(\hat{b} z_{++}+\hat{b}^{2} z_{+-}+z_{-+}+\hat{b} z_{--}\right), \\
& Z_{G^{\prime} \backslash e^{\prime},-u^{\prime},-v^{\prime}}(\lambda, \hat{b})=\hat{b}^{2} z_{++}+\hat{b}\left(z_{+-}+z_{-+}\right)+z_{--}
\end{aligned}
$$

Then, by Lemma 37, we can compute in poly-time the value of the ratio

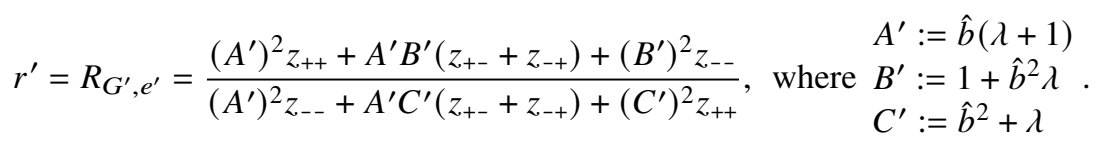

We are now in position to complete the computation of $r^{*}$. We first show how to decide in poly-time whether $z_{++}=0$. We claim that

$$
z_{++}=0 \Longleftrightarrow r=B / C \text { and } r^{\prime}=B^{\prime} / C^{\prime}
$$


Indeed, if $z_{++}=0$, then $z_{--}=0$ from Lemma 34 and, therefore, from (35), (36) we have that $r=B / C$ and $r^{\prime}=B^{\prime} / C^{\prime}$. Conversely, using that $A^{2} \neq B C$ and $\left(A^{\prime}\right)^{2} \neq B^{\prime} C^{\prime}$, we have that

$$
r=B / C \Longrightarrow C z_{++}=B z_{--}, \quad r^{\prime}=B^{\prime} / C^{\prime} \Longrightarrow C^{\prime} z_{++}=B^{\prime} z_{--},
$$

which together imply that $z_{++}=0$.

Using (37), we can decide in poly-time whether $z_{++}=0$. If so, by Lemma 34, we have $z_{--}=0$ and hence $r^{*}=\hat{b}$. So, assume $z_{++} \neq 0$, and hence $z_{--} \neq 0$ in what follows. We claim that

$$
z_{+-}+z_{-+}=0 \Longleftrightarrow r=\frac{A^{2} z_{++}+B^{2} z_{--}}{A^{2} z_{--}+C^{2} z_{++}}, r^{\prime}=\frac{\left(A^{\prime}\right)^{2} z_{++}+\left(B^{\prime}\right)^{2} z_{--}}{\left(A^{\prime}\right)^{2} z_{--}+\left(C^{\prime}\right)^{2} z_{++}} .
$$

The forward direction is again trivial. For the backward direction, we have

$$
\begin{aligned}
r & =\frac{A^{2} z_{++}+B^{2} z_{--}}{A^{2} z_{--}+C^{2} z_{++}} \Longrightarrow C z_{++}=B z_{--} \text {or } z_{+-}+z_{-+}=0, \\
r^{\prime} & =\frac{\left(A^{\prime}\right)^{2} z_{++}+\left(B^{\prime}\right)^{2} z_{--}}{\left(A^{\prime}\right)^{2} z_{--}+\left(C^{\prime}\right)^{2} z_{++}} \Longrightarrow C^{\prime} z_{++}=B^{\prime} z_{--} \text {or } z_{+-}+z_{-+}=0 .
\end{aligned}
$$

Since $z_{++}, z_{--} \neq 0$, we therefore obtain that $z_{+-}+z_{-+}=0$, proving (38).

Note that we can decide the right-hand side of (38) in poly-time using the value of the ratio $r^{\prime \prime}=z_{--} / z_{++}$from the second part of Lemma 37. If it turns out that $z_{+-}+z_{-+}=0$, then $r^{*}=1$ and we are done. Otherwise, we can use the values of $r$ and $r^{\prime \prime}$ to compute the ratios $\frac{z_{++}}{z_{+-}+z_{-+}}, \frac{z_{--}}{z_{+-}+z_{-+}}$, which we can then use to compute $r^{*}$.

This completes the computation of the ratio $r^{*}$ and therefore the proof of Theorem 1 .

\section{Equivalence for $\lambda=-1$ with Approximately Counting Perfect Matchings}

In this section, we show that for $\lambda=-1$, the problem of approximating the partition of the ferromagnetic Ising model on graphs of maximum degree $\Delta$ is equivalent to the problem \#PerfectMatchings, the problem of approximately counting perfect matchings on general graphs. The proof follows the technique in [19], where the case of negative $b$ but $\lambda=1$ was considered; here, however, we need to rework the relevant ingredients. The main such ingredient is the following 'high-temperature' expansion formula for $\lambda=-1$.

Lemma 39. Let $\lambda=-1$ and $b \neq-1$ be an arbitrary number. Then, for any graph $G=(V, E)$,

$$
Z_{G}(\lambda, b)=(-2)^{|V|}\left(\frac{1+b}{2}\right)^{|E|} \sum_{S \subseteq E ; \text { Sodd }}\left(\frac{1-b}{1+b}\right)^{|S|},
$$

where the sum is over $S \subseteq E$ such that every vertex $v \in V$ has odd degree in the subgraph $(V, S)$.

Proof. Let $G=(V, E)$ be a graph. For a set $S \subseteq E$ and a vertex $v \in V$, we let $d_{v}(S)$ denote the degree of $v$ in the subgraph $(V, S)$.

For the purposes of this proof, it will be convenient to view configurations of the Ising model on $G$ as vectors in $\{ \pm 1\}^{V}$. Now, for a configuration $\sigma \in\{ \pm 1\}^{V}$, we use the notation $n_{+}(\sigma)$ to denote the number of vertices with spin +1 . Observe that $n_{+}(\sigma)=\frac{1}{2}\left(|V|+\sum_{v \in V} \sigma_{v}\right)$ and that for an edge $e=(u, v)$, we have $b^{\mathbf{1}\left\{\sigma_{u} \neq \sigma_{v}\right\}}=\frac{1+b}{2}\left(1+\frac{1-b}{1+b} \sigma_{u} \sigma_{v}\right)=\rho\left(1+v \sigma_{u} \sigma_{v}\right)$, where for convenience we set $\rho:=\frac{1+b}{2}$ and $v:=\frac{1-b}{1+b}$. So, using that $\mathrm{i}^{2}=-1$,

$$
\begin{aligned}
Z_{G}(\lambda, b) & =\rho^{|E|} \sum_{\sigma \in\{ \pm 1\}^{V}} \lambda^{n_{+}(\sigma)} \prod_{e=(u, v) \in E}\left(1+v \sigma_{u} \sigma_{v}\right)=\rho^{|E|} \sum_{\sigma \rightarrow\{ \pm 1\}^{V}} \lambda^{n_{+}(\sigma)} \sum_{S \subseteq E} v^{|S|} \prod_{v \in V}\left(\sigma_{v}\right)^{d_{v}(S)} \\
& =\mathrm{i}^{|V|} \rho^{|E|} \sum_{S \subseteq E} v^{|S|} \sum_{\sigma \in\{ \pm 1\}^{V}} \prod_{v \in V} \mathrm{i}^{\sigma_{v}}\left(\sigma_{v}\right)^{d_{v}(S)} .
\end{aligned}
$$


The latter sum is equal to $\prod_{v \in V} \sum_{\sigma_{v} \in\{ \pm 1\}} \mathrm{i}^{\sigma_{v}}\left(\sigma_{v}\right)^{d_{v}(S)}$, which equals 0 if $d_{v}(S)$ is even and $2 \mathrm{i}$ otherwise. Plugging this in the expression above yields the lemma.

Now, we are ready to show the main theorem for this section. For counting problems $A, B$ we use the notion of AP-reductions; see [13]. Roughly, we have that $A \leq_{\mathrm{AP}} B$ if an FPRAS for $B$ can be converted to an FPRAS for $A$ and $A \equiv_{\mathrm{AP}} B$ if both $A \leq_{\mathrm{AP}} B$ and $B \leq_{\mathrm{AP}} A$ hold.

Theorem 40. Let $\lambda=-1$ and $b \in(0,1)$ be a rational. Then, for any connected graph $G$, we have $Z_{G}(\lambda, b)>0$ if $G$ has an even number of vertices and $Z_{G}(\lambda, b)=0$ otherwise.

Moreover, for all integers $\Delta \geq 3$, we have that \#lsingNorm $(\lambda, b, \Delta) \equiv_{\mathrm{AP}}$ \#PerfectMatchings.

Proof. The statement about the sign of $Z_{G}(\lambda, b)$ follows from Lemma 39 and the fact that every connected graph with an even number of vertices has a spanning subgraph where every vertex has odd degree. We thus focus on proving the AP-equivalence.

\#PerfectMatchings $\leq_{\mathrm{AP}}$ \#lsingNorm $(\lambda, \boldsymbol{b}, \Delta)$. It is well-known that the problem of approximating the number of perfect matchings on general graphs is AP-equivalent to the same problem on graphs of maximum degree 3; see, for example, [23, Lemma 28]. So, let $G=\left(V_{G}, E_{G}\right)$ be a graph of maximum degree 3, with $n=\left|V_{G}\right|$ and $m=\left|E_{G}\right|$, and let $\mathcal{M}$ be the set of perfect matchings of $G$. Since we can check whether a graph has a perfect matching in polynomial time, we may further assume that $|\mathcal{M}|>0$ and, in particular, that $n$ is even. Let $\epsilon \in(0,1)$ be the desired relative error that we want to approximate $|\mathcal{M}|$.

Analogous to (18) and (19), for $k=1+2\left\lceil\frac{m^{2}+\ln (1 / \epsilon)}{-\ln (1-b)}\right\rceil$, let $P_{k}=\left(V_{k}, E_{k}\right)$ be the path with $k$ vertices whose endpoints are labelled $u_{k}, v_{k}$ and $P_{k}^{*}=\left(V_{k}^{*}, E_{k}^{*}\right)$ be the graph obtained from $P_{k}$ by attaching a vertex $z_{i}$ to the $i$ th internal vertex $w_{i}$ of $P_{k}$, for $i=1, \ldots, k-2$. Let $A_{ \pm, \pm}:=Z_{P_{k}^{*}, \pm u_{k}, \pm v_{k}}(\lambda, b)$. Then, it is not hard to see that ${ }^{12}$

$$
\left[\begin{array}{cc}
A_{++} & -A_{+-} \\
-A_{-+} & A_{--}
\end{array}\right]=(1-b)^{k-2}\left[\begin{array}{ll}
1 & b \\
b & 1
\end{array}\right]^{k-1}
$$

and so

$$
\begin{aligned}
& A_{++}=A_{--}=\frac{1}{2}\left((1+b)^{k-1}+(1-b)^{k-1}\right)(1-b)^{k-2}, \text { and } \\
& A_{+-}=A_{-+}=\frac{1}{2}\left((1-b)^{k-1}-(1+b)^{k-1}\right)(1-b)^{k-2} .
\end{aligned}
$$

We next set

$$
b_{k}:=-\frac{A_{+-}}{A_{++}}=-\frac{A_{-+}}{A_{--}}, \quad \text { and observe that } \quad 1-(1-b)^{k-1}<b_{k}<1 .
$$

Let $H=\left(V_{H}, E_{H}\right)$ be an instance of \#IsingNorm $(\lambda, b, \Delta)$ obtained from $G$ by replacing every edge $e=(u, v)$ of $G$ with a distinct copy of $P_{k}^{*}$, identifying the endpoints $u, v$ with $u_{k}, v_{k}$, respectively. Then, we claim that

$$
Z_{H}(\lambda, b)=\left(A_{++}\right)^{m} Z_{G}\left(\lambda, b_{k}\right) .
$$

Indeed, for a configuration $\sigma: V_{G} \rightarrow\{+,-\}$, let $\Omega_{H, \sigma}=\left\{\sigma^{\prime}: V_{H} \rightarrow\{+,-\} \mid \sigma_{V_{G}}^{\prime}=\sigma\right\}$ be the configurations on $H$ which agree with $\sigma$ on $V_{G}$ and $Z_{H, \sigma}(\lambda, b)$ be the contribution to $Z_{H}(\lambda, b)$ from configurations in $\Omega_{H, \sigma}$. Then, we have

$$
Z_{H, \sigma}(\lambda, b)=\lambda^{\left|n_{+}(\sigma)\right|} \prod_{e=(u, v) \in E_{G}}(-1)^{\mathbf{1}_{\sigma_{u} \neq \sigma_{v}}} A_{\sigma_{u} \sigma_{v}}=\left(A_{++}\right)^{m} \lambda^{\left|n_{+}(\sigma)\right|} b_{k}^{\delta(\sigma)},
$$

\footnotetext{
${ }^{12}$ Here, the key observation is that for a configuration $\tau: V_{k} \rightarrow\{+,-\}$, the aggregate weight of configurations $\sigma: V_{k}^{*} \rightarrow$ $\{+,-\}$ with $\sigma_{V_{k}}=\tau$ is $(-1)^{\mathbf{1}\left\{\tau_{u_{k}} \neq \tau_{v_{k}}\right\}}(1-b)^{k-2} w_{P_{k}, \tau}(1, b)$. Indeed, if $\tau\left(w_{i}\right)=+$, then the contribution of the edge $\left(w_{i}, z_{i}\right)$ and the external field on $z_{i}$ is $b+\lambda=b-1$, whereas if $\tau\left(w_{i}\right)=-$, the contribution is $1+b \lambda=1-b$. This, combined with the factor $\lambda^{n_{+}(\tau)}$ coming from the external fields on $V_{k}$, gives the factor $(-1)^{\mathbf{1}\left\{\tau_{u_{k}} \neq \tau_{v_{k}}\right\}}(1-b)^{k-2}$ above; the remaining contribution is just the weight of $\tau$ on $P_{k}$ when the external field of all vertices on $P_{k}$ is equal to 1 .
} 
proving (40). Note from Lemma 39 we have that

$$
Z_{G}\left(\lambda, b_{k}\right)=2^{n}\left(\frac{1+b_{k}}{2}\right)^{m} \sum_{S \subseteq E ; S \text { odd }}\left(\frac{1-b_{k}}{1+b_{k}}\right)^{|S|} .
$$

Perfect matchings in $G$ are in 1-1 correspondence with odd sets $S \subseteq E$ with $|S|=n / 2$. Moreover, for any other odd set $S \subseteq E$ we have $|S|>n / 2+1$ and hence, using also (40), we obtain

$$
\left|\frac{Z_{H}(\lambda, b)}{\left(A_{++}\right)^{m} 2^{n}\left(\frac{1+b_{k}}{2}\right)^{m}\left(\frac{1-b_{k}}{1+b_{k}}\right)^{n / 2}}-\right| \mathcal{M}|| \leq 2^{m}\left(\frac{1-b_{k}}{1+b_{k}}\right) \leq \epsilon|\mathcal{M}| .
$$

Therefore, using an FPRAS for \#lsingNorm $(\lambda, b, \Delta)$, we can approximate $Z_{H}(\lambda, b)$ within relative error $\epsilon$ in time $\operatorname{poly}(n, 1 / \epsilon)$ and therefore compute $|\mathcal{M}|$ within relative error $\epsilon$, finishing the AP-reduction.

\#IsingNorm $(\lambda, \boldsymbol{b}, \Delta) \leq_{\mathrm{AP}}$ \#PerfectMatchings. We first consider the case $\Delta=3$. Let $G=(V, E)$ be a graph of maximum degree $\Delta=3$ that is input to \#IsingNorm $(\lambda, b, \Delta)$ and set $n=|V|, m=|E|$. We may assume that $n$ is even, since otherwise we can output 0 for the partition function. By Lemma 39 we have that

$$
Z_{G}(\lambda, b)=2^{n}\left(\frac{1+b}{2}\right)^{m} \sum_{S \subseteq E ; S \text { odd }}\left(\frac{1-b}{1+b}\right)^{|S|} .
$$

To formulate this in terms of perfect matchings, we construct a graph $G^{\prime}=\left(V^{\prime}, E^{\prime}\right)$ as follows, resembling the construction in [14]. For $v \in V$, let $d_{v}$ be the degree of $v$ in $G$. For a vertex $v \in V$, if $d_{v}=3$, replace $v$ with a triangle of vertices $T_{v}=\left\{v_{1}, v_{2}, v_{3}\right\}$; otherwise, keep $v$ in $G^{\prime}$ as well and let for convenience $T_{v}=\{v\}$. For every edge $(u, v) \in E$, add an edge in $G^{\prime}$ between a vertex in $T_{u}$ and $T_{w}$ so that $G^{\prime}$ has maximum degree 3; note that edges of $G$ that are not incident to degree-3 vertices belong to $G^{\prime}$ as well. We call internal all edges of $G^{\prime}$ whose endpoints belong to some $T_{v}$ and external all other edges of $G^{\prime}$. Note that an edge $e$ of $G$ maps to an external edge ex $(e)$ of $G^{\prime}$ bijectively under the natural mapping. We use $\operatorname{ex}\left(G^{\prime}\right)$ to denote the external edges of $G^{\prime}$.

For $v \in V$, observe that any perfect matching in $G^{\prime}$ must contain exactly one external edge incident to a vertex in $T_{v}$ if $\left|T_{v}\right|=1$ and two or three edges if $\left|T_{v}\right|=3$, either one internal and one external or three external, respectively. Based on this, we have that a perfect matching $M^{\prime}$ in $G^{\prime}$ maps bijectively to an odd subset $S$ of $G$, by adding an edge $e$ of $G$ to $S$ iff ex $(e) \in M^{\prime}$. Therefore, with $\mathcal{M}^{\prime}$ denoting the set of perfect matchings in $G^{\prime}$, we can rewrite (42) as

$$
Z_{G}(\lambda, b)=2^{n}\left(\frac{1+b}{2}\right)^{m} \sum_{M^{\prime} \in \mathcal{M}^{\prime}}\left(\frac{1-b}{1+b}\right)^{\left|M^{\prime} \cap \operatorname{ex}\left(G^{\prime}\right)\right|} .
$$

Let $n^{\prime}=\left|V^{\prime}\right| \leq 3 n$ and $m^{\prime}=\left|E^{\prime}\right|$. Let $p, q$ be positive integers with $\operatorname{gcd}(p, q)=1$ such that $\frac{p}{q}=\frac{1-b}{1+b}$. Let $G^{\prime \prime}$ be the multigraph obtained from $G^{\prime}$ by replacing every external edge $e=(u, v)$ with $p$ parallel edges connecting $u$ to a new vertex $w_{e}, q$ parallel edges connecting $w_{e}$ to a new vertex $z_{e}$ and an edge between $z_{e}$ and $v$; note that internal edges of $G^{\prime}$ are left intact. Let $\mathcal{M}^{\prime}$ and $\mathcal{M}^{\prime \prime}$ be the set of perfect matchings of $G^{\prime}$ and $G^{\prime \prime}$ Then, there is a one-to-many correspondence between perfect matchings $M^{\prime} \in \mathcal{M}^{\prime}$ in $G^{\prime}$ and perfect matchings $M^{\prime \prime} \in \mathcal{M}^{\prime \prime}$, where an internal edge $e$ is matched in $M^{\prime}$ iff $e$ is matched in $M^{\prime \prime}$, while an external edge $e=(u, v)$ is matched in $M^{\prime}$ iff $\left(z_{e}, v\right)$ is matched in $M^{\prime \prime}$. Note that, for an external edge $e$ and a perfect matching $M^{\prime \prime}$ of $G^{\prime \prime}$, if $\left(z_{e}, v\right)$ belongs to $M^{\prime \prime}$, then $u$ must be matched by one of the $p$ parallel edges connecting $u$ to $w_{e}$, whereas if $\left(z_{e}, v\right)$ does not belong to $M^{\prime \prime}$, $w_{e}$ and $z_{e}$ must be matched by one of the $q$ parallel edges connecting $u$ to $w_{e}$. It follows that

$$
\left|\mathcal{M}^{\prime \prime}\right|=\sum_{M \in \mathcal{M}^{\prime}} p^{\left|M \cap \operatorname{ex}\left(G^{\prime}\right)\right|} q^{m-\left|M \cap \operatorname{ex}\left(G^{\prime}\right)\right|} .
$$


Finally, if we let $G^{\prime \prime \prime}$ be the graph obtained from $G^{\prime \prime}$ by replacing every edge of $G^{\prime \prime}$ with a path of length 3, we have that the set of perfect matchings $\mathcal{M}^{\prime \prime \prime}$ off $G^{\prime \prime \prime}$ is in 1-1 correspondence with $\mathcal{M}^{\prime \prime}$ and we see that $2^{n}\left(\frac{1+b}{2}\right)^{m} q^{m}\left|\mathcal{M}^{\prime \prime \prime}\right|$ equals $Z_{G}(\lambda, b)$, completing the AP-reduction for $\Delta=3$.

To handle the case $\Delta \geq 4$, it suffices to show that \#Ising $\operatorname{Norm}(\lambda, b, \Delta) \leq_{\mathrm{AP}} \# \operatorname{lsing} \operatorname{Norm}(\lambda, b, 3)$, since AP-reductions are transitive; see [13]. Let $G=(V, E)$ be a graph of maximum degree $\Delta$ and set $n=|V|$. Let $V_{\leq 3}=\left\{v \in V \mid d_{v} \leq 3\right\}$ be the set of vertices in $G$ with degree $\leq 3$ and $V_{>3}$ be the set of the remaining vertices.

Construct a graph $G^{\prime}=\left(V^{\prime}, E^{\prime}\right)$ from $G$ by replacing every vertex $v \in V$ with $d_{v}=t \geq 4$, with a path of $2 t-1$ vertices if $t$ is odd and of $2 t-3$ vertices if $t$ is even. We partition the vertices on the path into two sets $T_{v}, T_{v}^{\prime}$ according to their parity, so that the endpoints of the path belong to $T_{v}$; note that $\left|T_{v}\right|=t$ if $t$ is odd, while $\left|T_{v}\right|=t-1$ if $t$ is even. We keep vertices $v \in V_{\leq 3}$ in $G^{\prime}$, and for such vertices, for convenience, we let $T_{v}=\{v\}$. Then, for every edge $(u, v) \in E$, we add an edge in $G^{\prime}$ between a vertex in $T_{u}$ and $T_{v}$ so that, in the end, $G^{\prime}$ has maximum degree 3 and, further, for vertices $v \in V_{>3}$ with $d_{v}$ even, exactly one endpoint of the path on $T_{v} \cup T_{v}^{\prime}$ has degree 3 in $G^{\prime}$ (and the other has degree 2). As before, we call an edge in $G^{\prime}$ internal if both of its endpoints lie within a set $T_{v}$ for some $v \in V$ and external otherwise.

The key observation is that the aggregate contribution to $Z_{G^{\prime}}(\lambda, b)$ from configurations on $G^{\prime}$ where, for some $v \in V$, the vertices in $T_{v}$ do not get the same spin is zero. ${ }^{13}$ For a configuration $\sigma$ on $G$, let $\Omega_{G^{\prime}, \sigma}$ be the set of configurations on $G^{\prime}$ such that all vertices in $T_{v}$ get the spin $\sigma_{v}$, and let $Z_{G^{\prime}, \sigma}(\lambda, b)$ be their aggregate contribution to $Z_{G^{\prime}}(\lambda, b)$, so that, from the observation above, we have

$$
Z_{G^{\prime}}(\lambda, b)=\sum_{\sigma: V \rightarrow\{+,-\}} Z_{G^{\prime}, \sigma}(\lambda, b) .
$$

For a configuration $\sigma: V \rightarrow\{+,-\}$, external edges and the external fields on $V_{\leq 3}$ contribute to $Z_{G^{\prime}, \sigma}(\lambda, b)$ a factor of $\lambda^{\left|n_{+}(\sigma) \cap V_{\leq 3}\right|} b^{\left|\delta_{G}(\sigma)\right|}$. For $v \in V_{>3}$ with $\sigma_{v}=+$, the edges in $T_{v} \cup T_{v}^{\prime}$ and the external fields on $T_{v} \cup T_{v}^{\prime}$ contribute to $Z_{G^{\prime}, \sigma}(\lambda, b)$ a factor of $-\left(1-b^{2}\right)^{\left|T_{v}\right|}$ and a factor of $\left(1-b^{2}\right)^{\left|T_{v}\right|}$ if $\sigma_{v}=-$. It follows that $Z_{G^{\prime}, \sigma}(\lambda, b)=\left(1-b^{2}\right)^{|T|} \lambda^{\left|n_{+}(\sigma)\right|} w_{G, \sigma}(\lambda, b)$ where $T=\cup_{v \in V ; d_{v} \geq 4}\left|T_{v}\right|$. It follows that

$$
Z_{G^{\prime}}(\lambda, b)=\left(1-b^{2}\right)^{|T|} Z_{G}(\lambda, b),
$$

therefore completing the AP-reduction, since by construction $G^{\prime}$ is a graph of maximum degree 3 .

This finishes the proof of Theorem 40.

Acknowledgements. We thank an anonymous referee for constructive feedback. PB is supported by the Netherlands Organisation of Scientific Research (NWO): 613.001.851. VP is supported by the Dutch Research Council (NWO) through the Gravitation Programme Networks (024.002.003). GR was (partly) supported by grant VI.Vidi.193.068 from the Dutch Research Council (NWO).

\section{Conflict of interest: None.}

\section{References}

[1] A. Barvinok, Combinatorics and Complexity of Partition Functions. Algorithms and Combinatorics (Springer International, Cham, 2017).

[2] A. Barvinok and G. Regts, 'Weighted counting of solutions to sparse systems of equations', Comb. Probab. Comput. 28(5) (2019), 696-719.

[3] A. F. Beardon, The Geometry of Discrete Groups, Vol. 91 of Graduate Texts in Mathematics (Springer, New York, 1995).

[4] F. Bencs, P. Buys, L. Guerini and H. Peters, 'Lee-Yang zeros of the antiferromagnetic Ising model', Ergod. Th. Dynam. Sys. (2021), 1-35. doi: 10.1017/etds.2021.25.

${ }^{13}$ This follows by observing that for a path with two edges, the aggregate weight of configurations where the endpoints of the path have different spins is equal to 0 (using that $\lambda=-1$ ). 
[5] F. Bencs, E. Davies, V. Patel and G. Regts, 'On zero-free regions for the anti-ferromagnetic Potts model on bounded-degree graphs', Ann. Inst. Henri Poincaré D 8(3) (2021), 459-489.

[6] I. Bezáková, A. Galanis, L. A. Goldberg and D. Štefankovič, 'Inapproximability of the independent set polynomial in the complex plane'. SIAM J. Comput. 49(5) (2020), STOC18-395-STOC18-448.

[7] I. Bezáková, A. Galanis, L. A. Goldberg and D. Štefankovič, 'The complexity of approximating the matching polynomial in the complex plane', ACM Trans. Comput. Theory 13(2) (2021), Art. 13, 37.

[8] M. J. Bremner, A. Montanaro and D. J. Shepherd, 'Average-case complexity versus approximate simulation of commuting quantum computations', Phys. Rev. Lett. 117 (2016), 080501.

[9] P. Buys, 'Cayley trees do not determine the maximal zero-free locus of the independence polynomial', Michigan Math. J. 70(3) (2021), 635-648.

[10] I. Chio, C. He, A. L. Ji and R. K. W. Roeder, 'Limiting measure of Lee-Yang zeros for the Cayley tree', Comm. Math. Phys. 370(3) (2019), 925-957.

[11] A. Collevecchio, T. M. Garoni, T. Hyndman and D. Tokarev, 'The worm process for the Ising model is rapidly mixing', $J$. Stat. Phys. 164(5) (2016), 1082-1102.

[12] G. De las Cuevas, W. Dür, M. Van den Nest and M. A. Martin-Delgado, 'Quantum algorithms for classical lattice models', New J. Phys. 13(9) (2011), 093021.

[13] M. Dyer, L. A. Goldberg, C. Greenhill and M. Jerrum, 'The relative complexity of approximate counting problems', Algorithmica 38(3) (2004), 471-500.

[14] M. E. Fisher, 'On the dimer solution of planar Ising models', J. Math. Phys. 7(10) (1966), 1776-1781.

[15] A. Galanis, L. A. Goldberg and A. Herrera-Poyatos, 'The complexity of approximating the complex-valued Potts model', Preprint, 2020, arXiv e-prints, abs/2005.01076.

[16] A. Galanis, D. Štefankovič and E. Vigoda, 'Inapproximability for antiferromagnetic spin systems in the tree nonuniqueness region', J. ACM 62(6) (2015), 60.

[17] A. Galanis, D. Štefankovič and E. Vigoda, 'Inapproximability of the partition function for the antiferromagnetic Ising and hard-core models', Comb. Probab. Comput. 25(4) (2016), 500-559.

[18] L. A. Goldberg and H. Guo, 'The complexity of approximating complex-valued Ising and Tutte partition functions', Comput. Complex. 26(4) (2017), 765-833.

[19] L. A. Goldberg and M. Jerrum, 'Inapproximability of the Tutte polynomial,' Inform. Comput. 206(7) (2008), 908-929.

[20] L. A. Goldberg and M. Jerrum, 'The complexity of computing the sign of the Tutte polynomial', SIAM J. Comput. 43(6) (2014), 1921-1952.

[21] H. Guo and P. Lu, 'Uniqueness, spatial mixing, and approximation for ferromagnetic 2-spin systems', ACM Trans. Comput. Theory 10(4) (2018), 25.

[22] H. Guo and M. Jerrum, 'Random cluster dynamics for the Ising model is rapidly mixing', Ann. Appl. Probab. 28(2) (2018), 1292-1313.

[23] H. Guo, C. Liao, P. Lu and C. Zhang, 'Zeros of Holant problems: locations and algorithms', in Proc. 30th Annual ACM-SIAM Sympos. Discrete Algorithms (Philadelphia, 2019), 2262-2278.

[24] H. Guo, J. Liu and P. Lu, 'Zeros of ferromagnetic 2-spin systems', in Proc. 2020 ACM-SIAM Sympos. Discrete Algorithms (Philadelphia, 2020), 181-192.

[25] O. J. Heilmann and E. H. Lieb, 'Theory of monomer-dimer systems', Comm. Math. Phys. 25(3) (1972), $190-232$.

[26] M. Jerrum and A. Sinclair, 'Polynomial-time approximation algorithms for the Ising model', SIAM J. Comput. 22(5) (1993), 1087-1116.

[27] M. R. Jerrum, L. G. Valiant and V. V. Vazirani, 'Random generation of combinatorial structures from a uniform distribution', Theoret. Comput. Sci. 43 (1986), 169-188.

[28] M. Kowalczyk and J.-Y. Cai, 'Holant problems for 3-regular graphs with complex edge functions', Theory Comput. Syst. 59(1) (2016), 133-158.

[29] T.-D. Lee and C.-N. Yang, 'Statistical theory of equations of state and phase transitions. I. Theory of condensation,' Phys. Rev. 87(3) (1952), 404.

[30] T.-D. Lee and C.-N. Yang, 'Statistical theory of equations of state and phase transitions. ii. Lattice gas and Ising model', Phys. Rev. 87(3) (1952), 410.

[31] L. Li, P. Lu and Y. Yin, 'Correlation decay up to uniqueness in spin systems', in Proc. 24th Annual ACM-SIAM Sympos. Discrete Algorithms (SODA) (Philadelphia, 2013), 67-84.

[32] J. Liu, P. Lu and C. Zhang, 'The complexity of ferromagnetic two-spin systems with external fields', in Approximation, Randomization, and Combinatorial Optimization. Algorithms and Techniques (APPROX/RANDOM 2014), Vol. 28 (Dagstuhl, 2014), 843-856.

[33] J. Liu, A. Sinclair and P. Srivastava, 'A deterministic algorithm for counting colorings with 2-Delta colors', in IEEE 60th Annual Sympos. Foundations of Computer Science (FOCS 2019) (2019), 1380-1404.

[34] J. Liu, A. Sinclair and P. Srivastava, 'Fisher zeros and correlation decay in the Ising model', J. Math. Phys. 60(10) (2019), 103304.

[35] J. Liu, A. Sinclair and P. Srivastava, 'The Ising partition function: Zeros and deterministic approximation', J. Stat. Phys. 174(2) (2019), 287-315. 
[36] R. L. Mann and M. J. Bremner, 'Approximation algorithms for complex-valued Ising models on bounded degree graphs', Quantum 3 (2019), 162.

[37] P. Mendes and F. Oliveira, 'On the topological structure of the arithmetic sum of two Cantor sets', Nonlinearity 7(2) (1994), 329-343.

[38] J. Milnor, Dynamics in One Complex Variable, Vol. 160 of Annals of Mathematics Studies, third edition (Princeton University Press, Princeton, NJ, 2006).

[39] V. Patel and G. Regts, 'Deterministic polynomial-time approximation algorithms for partition functions and graph polynomials', SIAM J. Comput. 46(6) (2017), 1893-1919.

[40] X. Peng, H. Zhou, B.-B. Wei, J. Cui, J. Du and R.-B. Liu, 'Experimental observation of Lee-Yang zeros', Phys. Rev. Lett. 114 (2015), 010601.

[41] H. Peters and G. Regts, 'On a conjecture of Sokal concerning roots of the independence polynomial', Mich. Math. J. 68(1) (2019), 33-55.

[42] H. Peters and G. Regts, 'Location of zeros for the partition function of the Ising model on bounded degree graphs', J. Lond. Math. Soc. 101 (2020), 765-785.

[43] A. Schrijver, Theory of Linear and Integer Programming (John Wiley \& Sons, New York, 1986).

[44] S. Shao and Y. Sun, 'Contraction: a unified perspective of correlation decay and zero-freeness of 2-spin systems', J. Stat. Phys. 185(2) (2021), Paper No. 12.

[45] A. Sinclair, P. Srivastava and M. Thurley, 'Approximation algorithms for two-state anti-ferromagnetic spin systems on bounded degree graphs', J. Stat. Phys. 155(4) (2014), 666-686.

[46] A. Sly and N. Sun, 'Counting in two-spin models on d-regular graphs', Ann. Probab. 42(6) (2014), $2383-2416$.

[47] L. G. Valiant, 'The complexity of computing the permanent', Theor. Comput. Sci. 8(2) (1979), 189-201. 